\title{
Neural dynamics of 1-D and 2-D brightness perception: A unified model of classical and recent phenomena
}

\author{
STEPHEN GROSSBERG \\ Boston University, Boston, Massachusetts \\ and \\ DEJAN TODOROVIĆ \\ Boston University, Boston, Massachusetts \\ and Univerzitet u Beogradu, Belgrade, Yugoslavia
}

\begin{abstract}
Computer simulations of a neural network model of 1-D and 2-D brightness phenomena are presented. The simulations indicate how configural image properties trigger interactions among spatially organized contrastive, boundary segmentation, and filling-in processes to generate emergent percepts. They provide the first unified mechanistic explanation of this set of phenomena, a number of which have received no previous mechanistic explanation. Network interactions between a Boundary Contour (BC) System and a Feature Contour (FC) System comprise the model. The BC System consists of a hierarchy of contrast-sensitive and orientationally tuned interactions, leading to a boundary segmentation. On and off geniculate cells and simple and complex cortical cells are modeled. Output signals from the BC System segmentation generate compartmental boundaries within the FC System. Contrast-sensitive inputs to the FC System generate a lateral filling-in of activation within FC System compartments. The filling-in process is defined by a nonlinear diffusion mechanism. Simulated phenomena include network responses to stimulus distributions that involve combinations of luminance steps, gradients, cusps, and corners of various sizes. These images include impossible staircases, bull's-eyes, nested combinations of luminance profiles, and images viewed under nonuniform illumination conditions. Simulated phenomena include variants of brightness constancy, brightness contrast, brightness assimilation, the Craik-O'Brien-Cornsweet effect, the Koffka-Benussi ring, the Kanizsa-Minguzzi anomalous brightness differentiation, the Hermann grid, and a Land Mondrian viewed under constant and gradient illumination that cannot be explained by retinex theory.
\end{abstract}

\section{PART 1 \\ INTRODUCTION: INTERACTIONS BETWEEN FORM AND APPEARANCE}

The sensitivity to ambient differences in light energy is the most basic discriminative ability of visual systems. The distribution of light energy reaching an animal's eyes is often characterized by regions of slow or zero gradients bordered by abrupt changes such as edges or contours. Correspondingly, one important tradition of psychophysical investigation has intensively studied the perceptual properties of juxtaposed homogeneous regions, leading

S.G.'s work was supported in part by the Air Force Office of Scientific Research (AFOSR F49620-87-C-0018 and AFOSR F49620-86-C0037) and the Army Research Office (ARO DAAG-29-85-K-0095). D.T.'s work was supported in part by the Army Research Office (ARO DAAG-29-85-K-0095). The authors thank Cynthia Suchta and Carol Yanakakis for their valuable assistance in the preparation of the manuscript and illustrations. S.G.'s mailing address is Center for Adaptive Systems, Boston University, 111 Cummington Street, Boston, MA 02215. D.T.'s mailing address is Filozofski Fakultet, Čika Ljubina 18 20, 11000 Belgrade, Yugoslavia. to such classical contributions as Weber's ratio and Fechner's law (Fechner, 1889), Metzger's Ganzfeld (Metzger, 1930), and the analysis of brightness constancy and contrast (Hess \& Pretori, 1894; Katz, 1935). A parallel line of psychophysical investigation has emphasized the processing of luminance discontinuities, notably edges and textures (Beck, 1966a, 1966b; Julesz, 1971; Ratliff, 1965).

Each type of investigation has provided essential data and concepts about visual perception, but, taken in isolation, each is nonetheless inherently incomplete. For example, the output of an edge-processing model produces only an outline of its visual environment and provides insufficient information about either the form or the appearance of the structures within the outline.

The nature of the incompleteness of vision concepts and models that focus on only one type of process at the expense of the other can be understood from two different perspectives. On the one hand, there exist large data bases which support the hypothesis that the processes that control the perception of form and appearance strongly interact before generating a final percept. Data concerning 


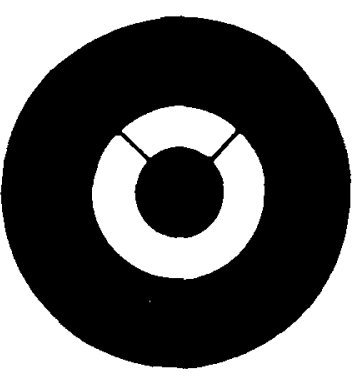

Figure 1. The Kanizsa-Minguzzi anomalous brightness differentiation. The bright annulus is divided into two unequal segments. The smaller segment looks slightly brighter.

one-dimensional (1-D) and 2-D brightness perception provide a particularly rich and constraining set of phenomena of this type. A number of key phenomena from this data base are given a unified explanation herein. In our model these brightness phenomena are generated as emergent properties of a neural network theory of preattentive visual perception (Cohen \& Grossberg, 1984; Grossberg, 1987a, 1987b; Grossberg \& Mingolla, 1985a, 1985b, 1987, 1988).

The anomalous brightness differentiation (Kanizsa \& Minguzzi, 1986) that is induced by the image shown in Figure 1 is one of the many brightness phenomena that can be explained by this theory. As Kanizsa and Minguzzi (1986) have noted, "this unexpected effect is not easily explained. In fact, it cannot be accounted for by any simple physiological mechanism such as lateral inhibition or frequency filtering. Furthermore, it does not seem obvious to invoke organizational factors, like figural belongingness of figure-ground articulation" (p. 223). We agree with these authors, but also show that this brightness phenomenon can be explained by the same theory that we use to explain many other brightness phenomena.

The properties of this theory clarify a deeper sense in which models that consider only form or appearance are incomplete. The perceptual theory that we apply suggests that each of the neural systems that process form or appearance compensates for limitations of the other systems with which it interacts. In other words, complete articulation of the processing rules for either system requires an analysis of the processing rules of the other system and of how the systems offset each other's complementary inadequacies through their interactions. Such an analysis has led to the identification of several new uncertainty principles which these systems overcome through parallel and hierarchical interactions (Grossberg, 1987a, 1987b).

The theory suggests that two parallel contour-sensitive processes interact to generate a percept of brightness. The Boundary Contour (BC) System, defined by a network hierarchy of oriented interactions, synthesizes an emergent binocular boundary segmentation from combinations of oriented and unoriented scenic elements. The Feature Contour (FC) System triggers a diffusive filling-in of featural quality within perceptual domains whose bound- aries are determined by output signals from the BC System. Neurophysiological and anatomical data from lateral geniculate nucleus and visual cortex which have been analyzed and predicted by the theory are summarized in Grossberg (1987a, 1987b).

Herein we use a simplified version of the model to explain brightness data. The simplified model does not include BC System and FC System mechanisms of emergent segmentation, multiple scale filtering, binocular interactions, and double-opponent processing. We focus on that large domain of brightness data whose qualitative properties can be explained by a single-scale, monocular version of the model. Our computer simulations of 1-D phenomena use a single set of numerical parameters, as do our simulations of 2-D brightness phenomena. We also show how parameter changes influence quantitative details of the simulation results. Since many visual images activate multiple spatial scales, binocular interactions, and emergent segmentations, our goal herein is to provide the type of quantitative understanding of model mechanisms that can achieve a unified qualitative explanation of difficult brightness data. The explanations of brightness phenomena within this reduced model are easily seen to be valid within the full theory, and to provide necessary information for future studies of quantitative matches between simulations and data in a multiple-scale, binocular setting.

The present article is organized as follows. In Part 2 we describe the neural network model that we use to simulate brightness phenomena. This model generalizes to two dimensions the types of processes that Cohen and Grossberg (1984) used to simulate 1-D brightness phenomena. This generalization conjoins processing concepts and mechanisms from Cohen and Grossberg (1984) and those from Grossberg and Mingolla (1985b, 1987). Part 3 defines and illustrates model properties through computer simulations of the model's reactions to a particular 2-D luminance distribution called the yin-yang square. The next two sections provide a unified account, through computer simulations, of several classical and recent varieties of brightness phenomena. Part 4 contains the 1-D simulations and Part 5 contains the 2-D simulations. Part 6 discusses how the model's concepts and mechanisms are related to other concepts and mechanisms of the theory which have been developed to analyze different data bases.

\section{PART 2 \\ THE MODEL: A HIERARCHY OF SPATIALLY ORGANIZED NETWORK INTERACTIONS}

Figure 2 provides an overview of the neural network model that we have analyzed. The model has six levels depicted as thick-bordered rectangles numbered from 1 to 6 . Levels 1 and 2 are preprocessing levels prior to the BC and FC Systems. Output signals from Level 2 generate inputs to both of these systems. Levels 3-5 are processing stages within the BC System. Level 6, which models 


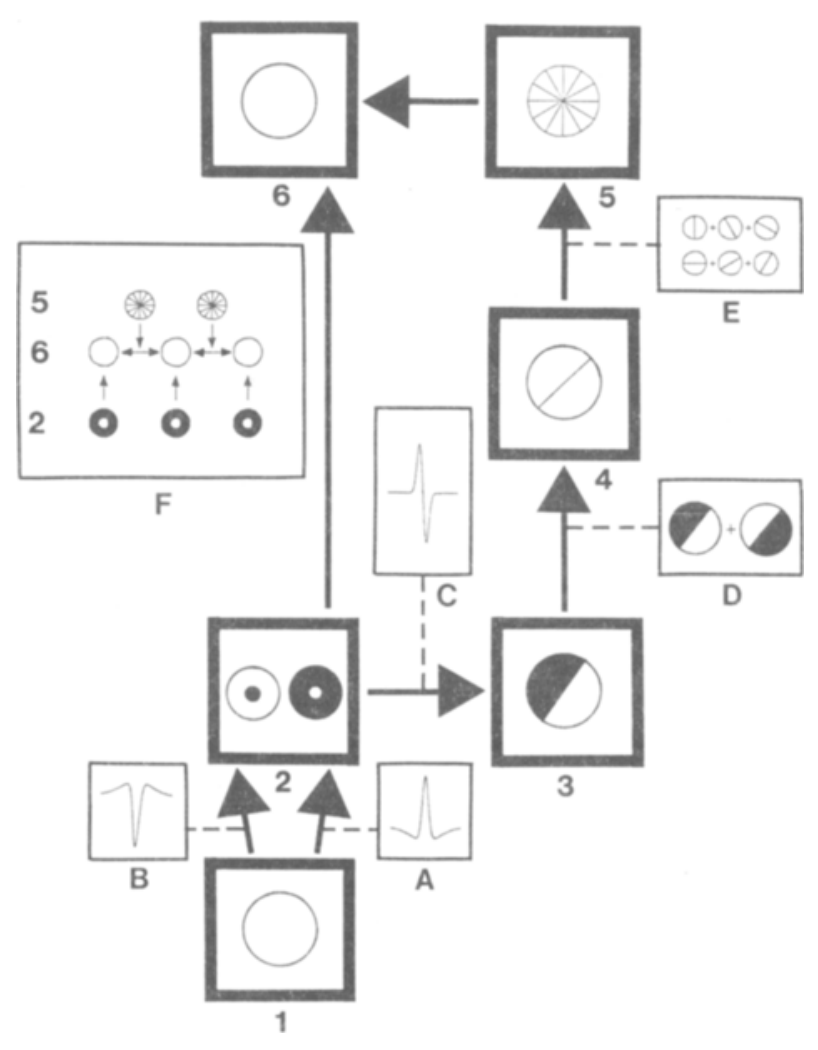

Figure 2. Overview of the model. The thick-bordered rectangles numbered from 1 to 6 correspond to the levels of the system. The symbols inside the rectangles are graphical mnemonics for the types of computational units residing at the corresponding model level. The arrows depict the interconnections between the levels. The thinbordered rectangles coded by letters $A$ through $E$ represent the type of processing between pairs of levels. Inset $F$ illustrates how the activity at Level 6 is modulated by outputs from Level 2 and Level 5 . See text for additional details.

the FC System, receives inputs from both Level 2 and Level 5.

Each level contains a different type of neural network. The type of network is indicated by the symbol inside the rectangle. The symbols provide graphical mnemonics for the processing characteristics at a given level, and are used in the figures that present the computer simulations of the 2-D implementation of the model. The arrows connecting the rectangles depict the flow of processing between the levels. The type of signal processing between different levels is indicated inside thin-bordered insets attached by dotted lines to appropriate arrows, and coded by letters $\mathrm{A}$ through $\mathbf{E}$. The sketch inside the inset coded $\mathrm{F}$ depicts the complex interactions between Levels 2,5 , and 6 . The properties of different levels and transformations will be discussed in detail in the following pages. To explain the working of the system, we repeatedly refer to Figure 2, and present a number of computer simulations of network dynamics. The mathematical equations of the model are described in the Appendix.

\section{Level 1: The Stimulus Distribution}

The first level of the model consists of a set of units that sample the luminance distribution. In the $1-\mathrm{D}$ version of the model, the units are arranged on a line; in the 2-D version, they form a square grid.

\section{Level 2: Circular Concentric On and Off Units (LGN Cells)}

Level 2 of the network models cells with the type of circular concentric receptive fields found at early levels of the visual system, such as ganglion retinal cells or lateral geniculate cells. These cells come in two varieties: the on-center-off-surround cells, or on-cells, and the offcenter-on-surround cells, or off-cells. In Figure 2, the on-cells are symbolized with a white center and a black annulus, and the off-cells with a black center and a white annulus. The mathematical specification of the receptive field (see the Appendix) uses feedforward shunting equations (Grossberg, 1983) because of their sensitivity to input reflectances. Thus, the model utilizes the simplest physiological mechanism that discounts the illuminant and is sufficient to explain key properties of the targeted brightness percepts.

The 1-D cross-sections of these receptive fields are presented in insets A and B in Figure 2. In two dimensions, these profiles have the shape of sombreros for onunits and inverted sombreros for off-units. The activity level of such cells correlates with the size of the centersurround luminance contrast. More luminance in the center than in the surround induces increased activity in on-cells and decreased activity in off-cells. Inverse luminance conditions result in inverse activation levels. Due to the shunting interaction, the cells are sensitive to relative contrast in a manner approximating a Weber law (Grossberg, 1983). In addition, the cells are tuned to display nonnegligible activity levels even for homogeneous stimulation, as do retinal ganglion cells (Enroth-Cugell \& Robson, 1984). This property enables such a cell to generate output signals that are sensitive to both excitatory and inhibitory inputs.

\section{Level 3: Oriented Direction-of-Contrast-Sensitive Units (Simple Cells)}

Level 3 consists of cell units that share properties with cortical simple cells. The symbol for these units in Figure 2 expresses their sensitivity to luminance contrast of a given orientation and given direction of contrast. Inset $C$ depicts the 1-D cross-section of the receptive field of such units, taken with respect to the network of on-cells.

In our 2-D simulations, the function we used to generate this receptive field profile was the difference of two identical bivariate Gaussians whose centers were shifted with respect to each other (see the Appendix). A similar formalization was used by Heggelund (1981a, 1985). As yet, neither anatomical nor physiological studies have unequivocally demonstrated the manner in which orienta- 
tional sensitivity arises in cortical cells (Braitenberg \& Braitenberg, 1979; Nielsen, 1985; Sillito, 1984). Our ambition was not to resolve this issue, but to find a simple, yet acceptable, arrangement that would realize the desired functional properties. In our current implementation, Level 3 units are activated by Level 2 on-units.

In order to represent a number of different orientation sensitivities, Level 3 consists of 12 different cell types, each sensitive to a different orientation and direction of contrast. A convenient "hour code" was used to denote these units. For example, a cell tuned to detect vertical left-to-right light-dark edges is denoted a " 12 o'clock unit," whereas a cell with the same axis orientation but reversed contrast preference is a "6 o'clock unit" (see Figure 7). In the 1-D implementation, only two directions were used.

\section{Level 4: Oriented Direction-of-Contrast-Insensitive Units (Complex Cells)}

Level 3 units are sensitive to oriented contrasts in a specific direction-of-contrast, as are cortical simple cells. However, complex cell units sensitive to contrasts of specific orientation regardless of contrast polarity are also well known to occur in striate cortical area 17 of monkeys (DeValois, Albrecht, \& Thorell, 1982; Gouras \& Krüger, 1979; Hubel \& Wiesel, 1968; Schiller, Finlay, \& Volman, 1976; Tanaka, Lee, \& Creutzfeldt, 1983) and cats (Heggelund, 1981b; Hubel \& Wiesel, 1962; Spitzer \& Hochstein, 1985). See Grossberg (1987a) for a review of relevant data and related models.

Units fulfilling the above criteria populate Level 4 of the network. Inset $D$ in Figure 2 depicts the construction of Level 4 cells out of Level 3 cells. The mathematical specification is similar to the one used by Grossberg and Mingolla (1985a, 1985b) and Spitzer and Hochstein (1985). The symbol of Level 4 units expresses their sensitivity to oriented contrasts of either direction. Each Level 4 unit at a particular location is excited by 2 Level 3 units at the corresponding location having the same axis of orientation but opposite direction preference. For example, a 3 o'clock unit and a 9 o'clock unit in Level 3 generate a horizontal contrast detector in Level 4 . Thus, the 12 Level 3 networks give rise to 6 Level 4 networks. Interestingly, several physiological studies have found that the simple cells outnumber the complex cells in a ratio of approximately 2 to 1 , and that complex cells have higher spontaneous activity levels than simple cells (Kato, Bishop, \& Orban, 1978). Both of these properties are consistent with the proposed circuitry.

\section{Level 5: Boundary Contour Units}

In the simulations presented in this paper, we have used a simplified version of the BC System. The final output of this system is located at Level 5 of the model. A unit at a given Level 5 location can be excited by any Level 4 unit located at the position corresponding to the position of the Level 5 unit. A Level 4 unit excites a Level 5 unit only if its own activity exceeds a threshold value. The pooling of signals sensitive to different orientations is sketched in inset $E$ and expressed in the symbol for Level 5 in Figure 2. This pooling may, in principle, occur entirely in convergent output pathways from the BC System to the FC System, rather than at a separate level of cells within the BC System.

\section{Level 6: Diffusive Filling-In Within a Cell Syncytium}

Network activity at Level 6 of our model corresponds to the brightness percept. Level 6 is part of the FC System, which is composed of a syncytium of cells. A syncytium of cells is a regular array of intimately connected cells such that contiguous cells can easily pass signals between each other's compartment membranes, possible via gap junctions (Piccolino, Neyton, \& Gerschenfeld, 1984). Due to the syncytial coupling of each cell with its neighbors, the activity can rapidly spread to neighboring cells, then to neighbors of the neighbors, and so on.

Because the spreading, or filling-in, of activation occurs via a process of diffusion, it tends to average the activation that is triggered by a FC input from Level 2 across the Level 6 cells that receive this spreading activity. This averaged activity spreads across the syncytium with a space constant that depends upon the electrical activities of both the cell interiors and their membranes. The electrical properties of the cell membranes can be altered by $\mathrm{BC}$ signals in the following way. A BC signal is assumed to decrease the diffusion constant of its target cell membranes within the cell syncytium. It does so by acting as an inhibitory gating signal that causes an increase in cellmembrane resistance. A BC signal hereby creates a barrier to the filling-in process at its target cells.

The inset labeled $F$ in Figure 2 summarizes the three factors that influence the magnitude of activity of units at Level 6. First, each unit receives bottom-up input from Level 2, the field of concentric on-cells. Second, there are lateral connections between neighboring units at Level 6 that define the syncytium, which enables withinnetwork spread of activation, or filling-in. Third, this lateral spread is modulated by inhibition from Level 5 in the form of BC signals capable of decreasing the magnitude of mutual influence between neighboring Level 6 units. The net effect of these interactions is that the FC signals generated by the concentric on-cells are diffused and averaged within boundaries generated by BC signals.

The idea of a filling-in process has been invoked in varous forms by several authors in discussions of different brightness phenomena (Davidson \& Whiteside, 1971; Fry, 1948; Gerrits \& Vendrik, 1970; Hamada, 1984; Walls, 1954). In the present model, this notion is fully formalized, related to a possible neurophysiological foundation, tied in with other mechanisms as a part of a more general vision theory, and applied in a systematic way to a variety of brightness phenomena. 


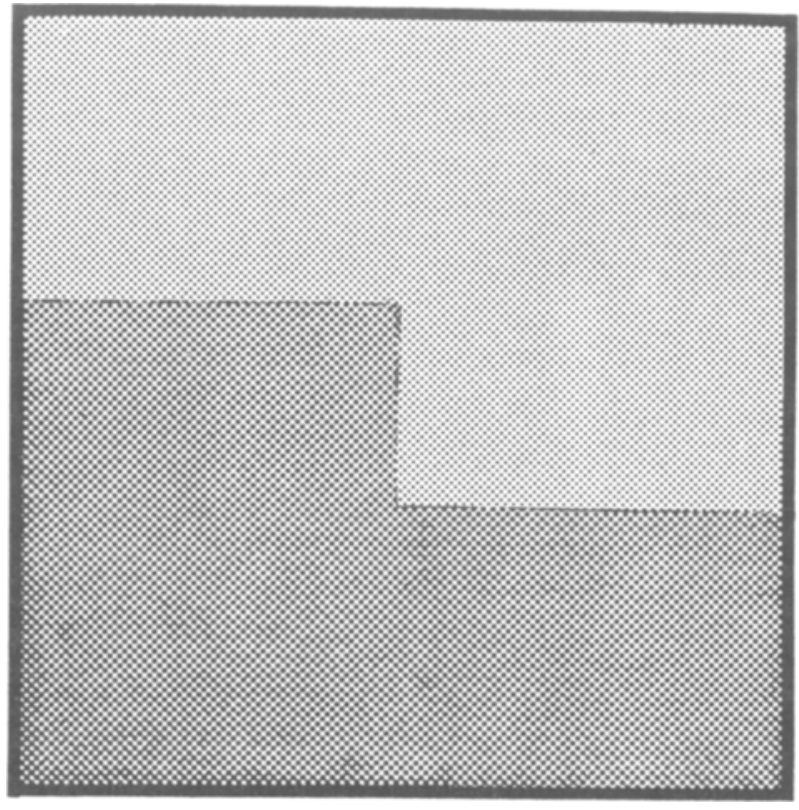

Figure 3. The yin-yang square. The $Z$-shaped luminance step separates two regions of different homogeneous luminance. This 2-D luminance distribution is used as an input to the model. Figures 4 through 9 present the activity profiles induced by this stimulus at various levels of the model. The equations and parameters used in the simulations are listed in the Appendix.

\section{PART 3}

\section{AN EXAMPLE OF SYSTEM DYNAMICS AND GRAPHICAL REPRESENTATION: THE YIN-YANG SQUARE}

Figure 3 shows an image that was used as input to the system to illustrate its functioning. It is a square version of the yin-yang symbol. The salient feature of the stimulus is the Z-shaped luminance contour that separates the top and right bright portion (yin) from the bottom and left dark portion (yang). Although the visual structure of the display is simple, it is sufficiently rich to illustrate a number of properties of our system.

The way this stimulus is represented in the system is shown in Figure 4. This representation, corresponding to Level 1 in Figure 2, has a number of features that are typical in our representations of 2-D patterns of activity at all levels of the system. First, it is a square $16 \times 16$ grid of units or cells. (Later, we will also present $30 \times 30$ and $40 \times 40$ examples, and 1-D representations in cases in which the 2-D structure is not essential for the analysis of the image.) Second, such a grid of units is only a sample from a potentially much denser and larger grid. This level of resolution and size proved, however, to represent adequately the activity patterns for the types of images we used. Third, the magnitude of activity of a unit is coded by the size of the graphical symbol representing the particular level of the system. In Figure 4, the radius of a circle is proportional to the magnitude of activity of the Level 1 unit corresponding to the grid position at the center of the circle. Since, in this case, the stimulus has only two intensity levels, circles of only two sizes appear in the representation. The larger circles correspond to the more luminant portion of the stimulus, the smaller ones to the less luminant portion, and their spatial arrangement corresponds to the spatial luminance structure of the stimulus.

The particular sizes of the circles on the printed page were chosen according to the following scaling procedure: The unit or units with the maximum activity are represented with circles whose radius is equal to half the distance between the centers of two neighboring units on the grid; the remaining circles are scaled proportionally. Most of the 2-D figures in the paper were scaled separately, with each graph scaled with respect to its own maximum. A common scale for a large set of figures was impracticable in view of many different stimulus distributions, model levels, and parameter ranges. Therefore, relative size comparisons are meaningful within a figure, but generally are not between figures. However, in some cases, which will be specifically noted, a common scale was used for several figures.

We considered other ways to represent 2-D activity profiles. For example, one could use 3-D graphs in which the $x$ - and $y$-coordinate code the spatial variables, and the $z$-coordinate codes strength of activity. Another possibility is to use a shading representation, in which the luminance level codes magnitude of activity. There are several reasons why we have not used such more standard formats. The detailed structure of activity profiles appeared to us in most cases to be easier to grasp in the symbol-size code than in the $z$-coordinate or shading code.

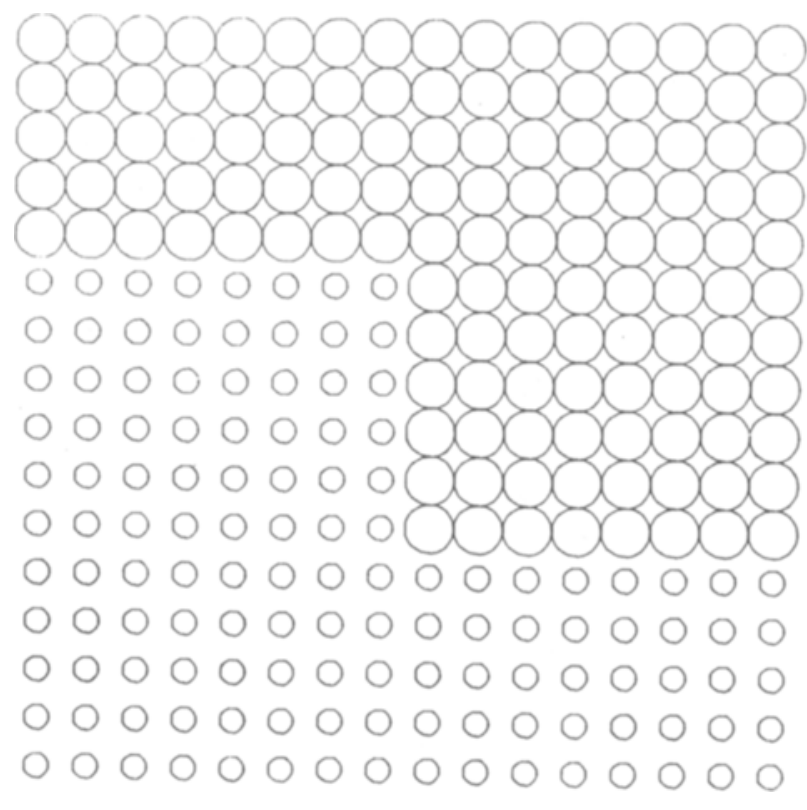

Figure 4. The representation of the yin-yang square at Level 1 of the model. Local luminance level is coded by the magnitude of the circle radius. 
The hidden-line-removal technique, used in some versions of the first code, unfortunately also hides some aspects of the profile structure. A shading representation seemed to us particularly awkward, since our purpose was to study the more subtle and illusory aspects of brightness perception. On the other hand, since a symbol has other features in addition to size, these other features can be used to code other aspects of activity profiles. As illustrated herein, the use of different mnemonic symbols for different levels of the system enhances the clarity of presentation of its structure and function. The advantages of a symbol-size representation become apparent in the representation of the Level 2 response to the yin-yang square.

Figures $5 \mathrm{a}$ and $5 \mathrm{~b}$ present computer simulations of activity profiles of Level 2 on-units and off-units, respectively, in response to the stimulus shown in Figure 3. These are $16 \times 16$ grids of units, in which symbol size codes activation strength. The shapes of these profiles are the result of two components: the structure of the stimulus and the structure of the networks. The interaction of these two factors results in field distributions in which the structure of the stimulus is recognizable, but distorted. The particular forms of these transformations will now be discussed in more detail.

In contrast to only two different magnitudes in the representation of the stimulus at Level 1 , the Level 2 equilibrium activity patterns of the on-units in Figure 5a exhibit many more magnitudes. In the stimulus, differences between adjacent units exist only along the contour, and they are all of the same size. In the on-unit response fields, the largest differences are also found along the contour, but they vary systematically in size. Furthermore, the response-strength gradient diminishes with increasing distance from the contour.

In addition, the extremal response values correspond to stimulus contour corners-that is, locations of abrupt change in the orientation of luminance contrast. This feature is a straightforward consequence of the structure of the stimulus transformation imposed by units with circular concentric antagonistic receptive fields. Thus, the location of the most activated unit in the on-field in Figure 5a corresponds to the convex bright corner of the yin portion of the stimulus distribution. The Level 2 onunit at this location receives in its receptive field center as much stimulation as do all other units in the bright portion of the stimulus. However, the surround of this unit contributes the least amount of inhibition, because almost three-quarters of its area lies in the concave dark corner of the yang portion of the stimulus distribution. Analogously, the minimum of the activity profile in the on-field is located in a position corresponding to the convex dark corner of the yang portion of the stimulus.

These features of the 2-D on-cell response profiles are consistent with well-known physiological results and theories involving reactions to 1-D luminance stepdistributions in the limulus (Ratliff \& Hartline, 1959) and the cat (Enroth-Cugell \& Robson, 1966). The 1-D

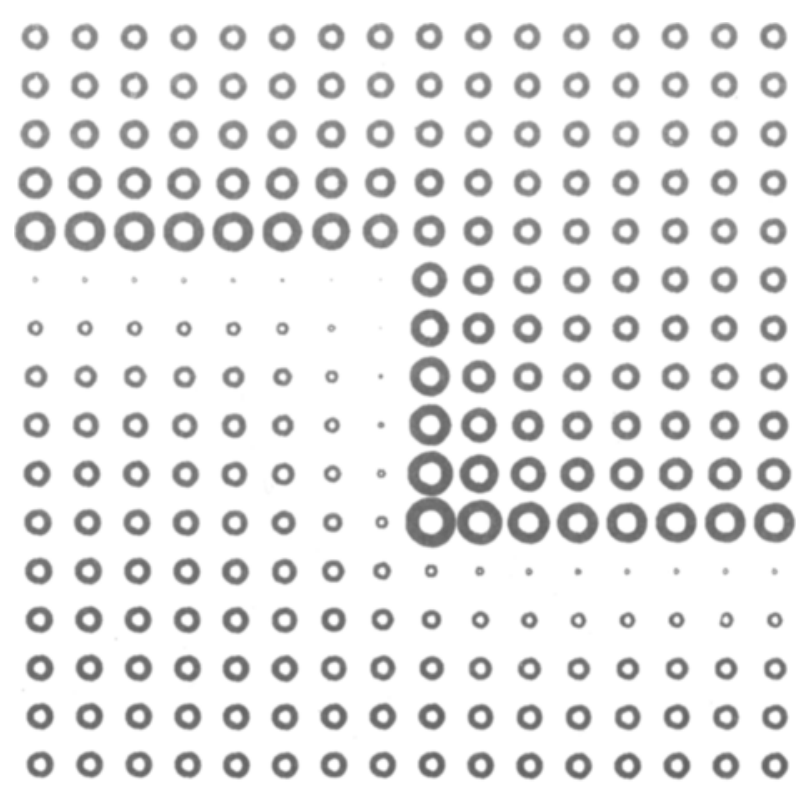

(a)

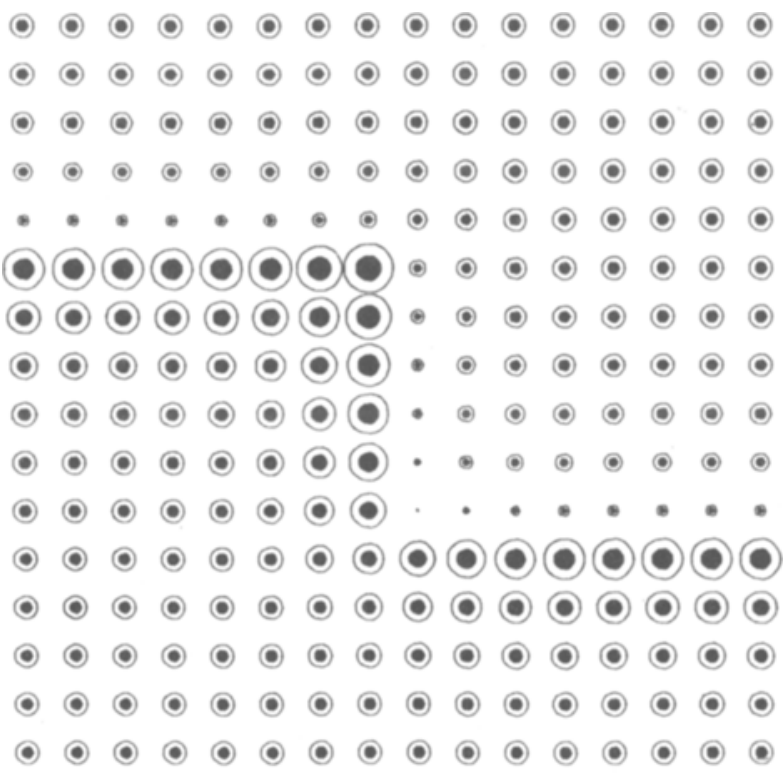

(b)

Figure 5. The Level 2 activity profiles. (a) The on-cell distribution. (b) The off-cell distribution. The extremal values in the two distributions correspond to the two corners of the yin-yang square. The complementarity of the two activity profiles reflects the complementary structure of the receptive fields of the on-cells and the off-cells. Neither of the two activity profiles can account for the brightness distribution. See text for details.

response profiles to such stimuli exhibit increases and decreases at the edges, resulting in cusp-shaped distributions. Such profiles can be found in many 1-D crosssections of Figure 5a. For example, consider the 16 leftmost units in the on-cell field, forming the left border of Figure 5a. Starting from the top left unit, and proceed- 
ing toward the bottom left unit, the activity level increases toward the luminance edge, drops abruptly, and returns gradually to a medium level. It is widely accepted that these overshoots and undershoots in physiological activity contribute to the phenomenon of Mach bands (Ratliff, 1965).

The corner-related extrema in the Level 2 distribution profiles may contribute to the enhanced brightness phenomena involving nested sets of corners that were analyzed by Hurvich (1981), who discovered them in paintings by Vasarely. Analogous effects were described by Todorović (1983) in some related visual situations. In analogy to Mach bands, they might be called "Mach corners." A corresponding physiological study has, to our knowledge, not been performed.

A comparison of the on-unit activity pattern in Figure 5a with the off-unit activity pattern in Figure 5b shows a symmetry, or duality, due to the complementary structure of the receptive fields of on-units and off-units. For example, the locus of minimum activity in Figure 5a corresponds to the locus of maximum activity in Figure $5 \mathrm{~b}$, and vice versa. The regions of activity overshoots and undershoots along stimulus contours have also exchanged locations. More generally, for any two units (and, in particular, for any two adjacent units), the following observation holds: If, in Figure 5a, the activity of the first unit is larger than the activity of the second one, then, for the two corresponding units in Figure $5 b$, the activity of the first unit will be smaller than the activity of the second one, and vice versa.

The final point with respect to the shapes of Level 2 profiles concerns the regions located at some distance from the Z-shaped contour. For example, consider the bottom left and the top right unit, whose locations are most removed from the contour region. Their activity level is approximately the same, both within the on-unit field and within the off-unit field. This equality in activity level contrasts with the appearance of the corresponding image portions (Figure 3): the lower left region of the image appears darker than the upper right region. This aspect of the brightness profile cannot be accounted for by the activity profile of cells with concentric antagonistic receptive fields, which are insensitive to differences of the $a b-$ solute level of homogeneous stimulation. In the model, the brightness of Figure 3 is accounted for by the Level 6 distribution.

For pictorial clarity, we first present computer simulations of the Level 3 activity patterns generated across cells with a single orientational preference (Figures 6a-6f; these figures were drawn using a common scale). Then we combine all of these results into a pictorial summary of the total Level 3 equilibrium response (Figure 8a). Figures 6 a through $6 \mathrm{~d}$ present activity profiles for four networks that contain 3 o'clock through 6 o'clock Level 3 units, respectively. There are several points about these simulations that are worth noting.

The total number of activated cells is smaller in each of these Level 3 simulations than in the Level 2 simula- tions. Although most Level 2 units show some activity, only a restricted set in each Level 3 network plays an active part in the detection of a BC. In contrast to what is found in Level 2 units, the net excitatory effect of the receptive fields in Level 3 cells balances the net inhibitory effect (see inset $\mathrm{C}$ ) in accord with physiological findings that cortical simple cells respond weakly, if at all, to homogeneous stimulation (Hubel \& Wiesel, 1968).

Due to the spatial scale and orientational tuning of Level 3 cells, different units prefer different image features. As expected, the 3 o'clock cells (Figure 6a) detect the horizontal edges of appropriate contrast direction, whereas the 6 o'clock cells (Figure 6d) register the vertical edge. However, the simulations also caution against a feature-detection notion that would hold that such cells respond uniquely to particular stimulus features. As has been pointed out in various forms before (Frisby, 1979; Sekuler, 1974), the activity of a unit does not uniquely correspond to the objective presence of a feature, or to our perception of such a feature. For example, in Figure 6d, the strongly activated column of 6 o'clock units is surrounded by a halo of weakly activated units. With larger receptive field size or, equivalently in our simulations, larger sample density, an even fuzzier swarm of vertical-edge-activated units emerges, as shown in Figure 6e. Such a spatially diffuse activity profile contrasts with the sharp localization of both the physical luminance edge and our percept of it.

The nonuniqueness of the relationship of stimulus features to unit activity is also shown in orientation preferences. Since each cell responds to a band of orientations, suboptimal stimuli can induce appreciable activity levels. Figures $6 \mathrm{~b}$ and $6 \mathrm{c}$ show that the 4 o'clock and 5 o'clock units respond reasonably well to both horizontal and vertical stimulus features. Consequently, the overall Z-shaped structure of the stimulus contour is better reflected by the profiles of the nonoptimally tuned units in Figures $6 \mathrm{~b}$ and $6 \mathrm{c}$ than by the profiles of the optimally tuned units in Figures $6 \mathrm{a}$ and $6 \mathrm{~d}$. In particular, the activity patterns of the 4 o'clock and 5 o'clock cells are sensitive to the existence of stimulus corners, which are absent in the activity patterns of the 3 o'clock and 6 o'clock cells.

Figure $6 \mathrm{f}$ presents the activity pattern across the field of 9 o'clock units. These cells have the same preferred orientation axis as the 3 o'clock units, but an inverse direction preference. The consequence is still another type of complementary relationship between response profiles: the regions of suprathreshold activity in Figure 6a almost exactly coincide with regions of zero activity in Figure 6f, and vice versa, although the maximal activity levels in Figure $6 \mathrm{f}$ are much smaller than those in Figure $6 \mathrm{a}$.

The activity pattern in Figure 6f may appear counterintuitive, because the horizontally oriented luminance contrast detected by these units is opposite, in direction, to the one present in the stimulus. However, recall that, in accord with visual anatomy, the Level 3 units are activated by the Level 2 on-unit activity pattern (Figure 5a) rather than by the image itself (Figure 3). In particular, 


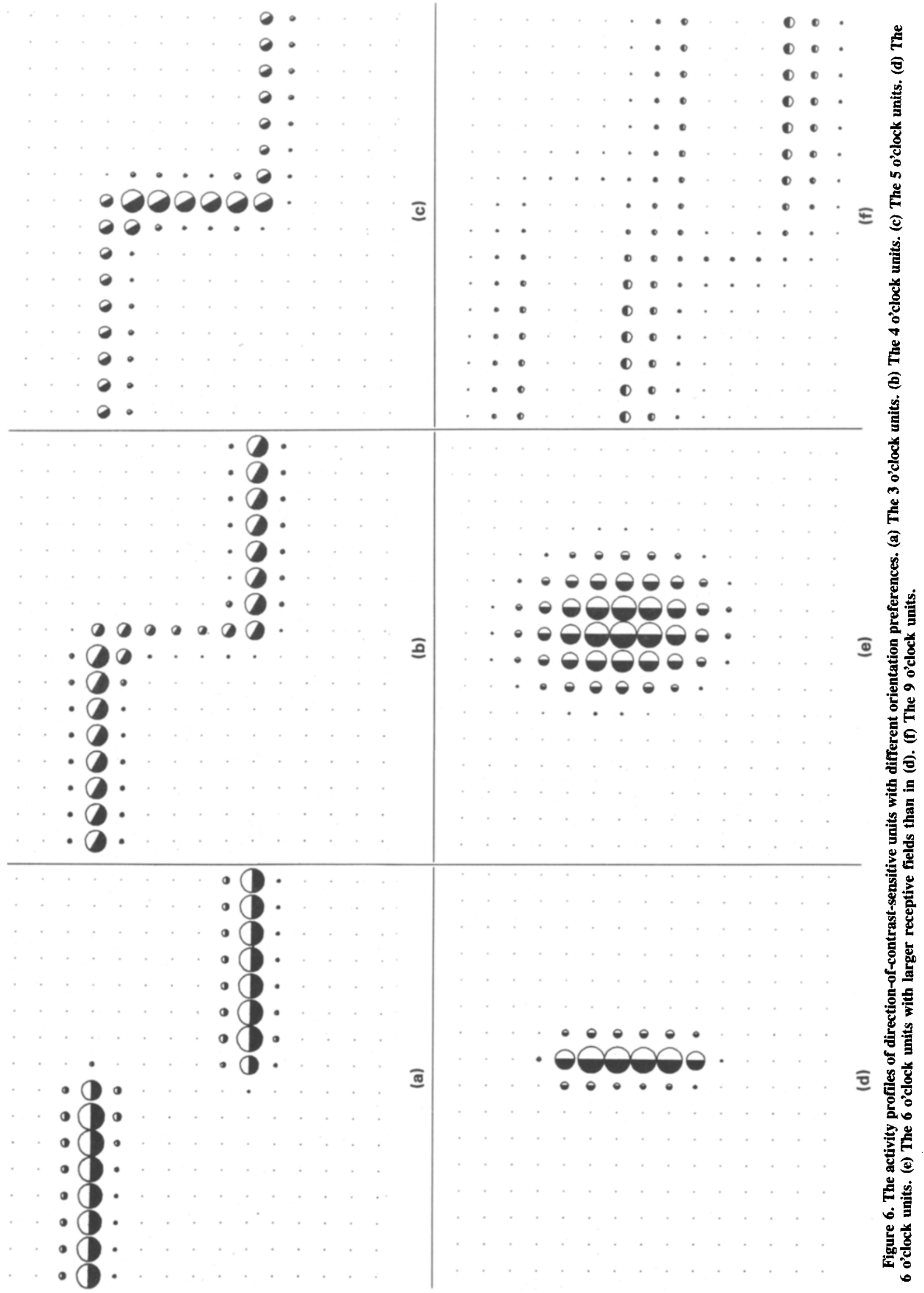



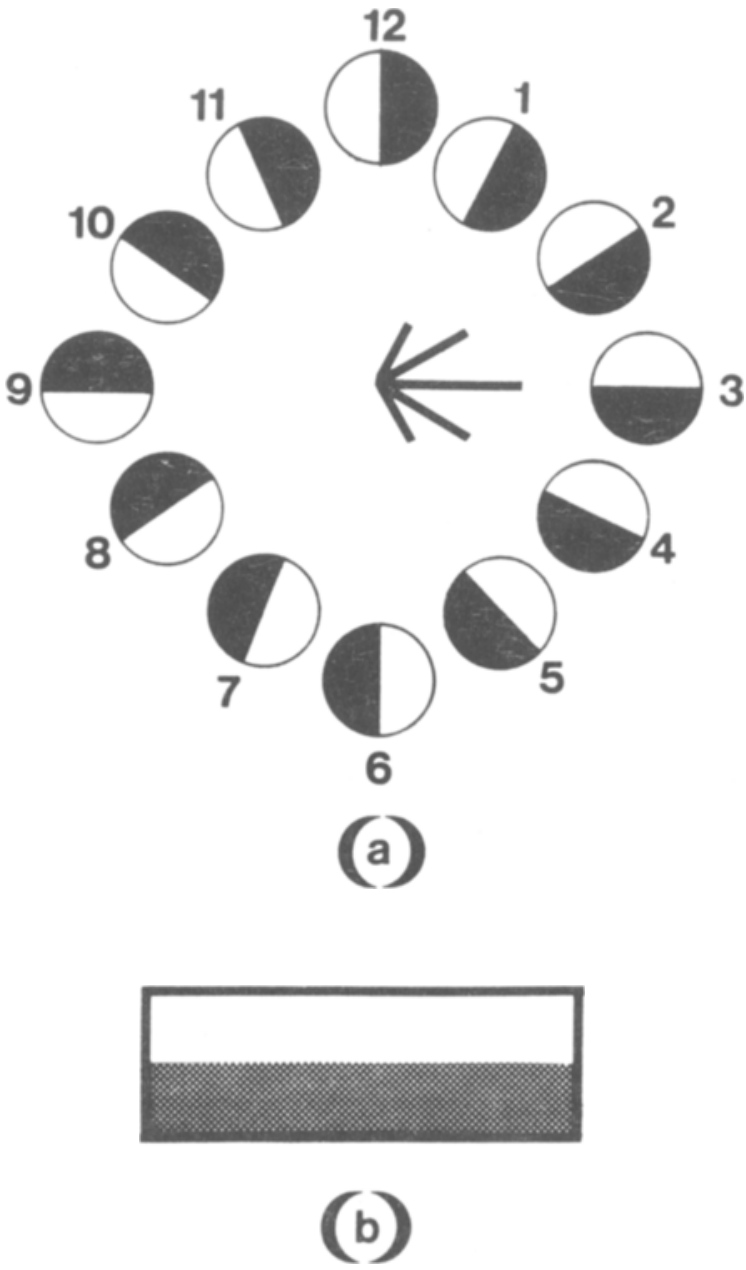

Figure 7. Units at Level 3 are symbolized by graphical mnemonics expressing their different orientation and contrast direction sensitivities. These symbols, which are used in Figure 6 to depict the activity profiles for units of a given sensitivity, are numbered according to the hour code. A different symbolism is used to represent the activity of all Level 3 units in a single graph. The activity level of a unit is coded by the length of the line pointing toward its hour code. The cluster of lines at the center of Figure 7a represents the activity of all units at a location responding to the horizontally oriented contrast depicted in Figure $7 b$.

moving from the top left unit toward the bottom left unit in Figure 5a, the activity rises gradually. This activity gradient is picked up by 9 o'clock cells in Figure 6f. Next, there is a sharp drop, strongly activating the 3 o'clock cells in Figure 6a. Finally, the activity gradient slowly rises and is again sensed by 9 o'clock cells. Since the average magnitude of unit activity in Figure $6 f$ is relatively small, it may in vivo be submerged in noise. The set of activity patterns in Figures 6a-6f provide qualitative predictions about the first few levels of the BC computation.

Figure 7a provides a concise symbolism for the representation of all 12 Level 3 activity patterns. To construct such a total response profile, we have used a representation format in which the size of the response is coded with the length of the clock hand pointing in the direction of the hour code of a unit. Figure $7 \mathrm{~b}$ depicts a horizontally oriented input to Level 3 , with stronger activation at the top than at the bottom strip. The response to such an input would be largest for the optimally tuned 3 o'clock unit, weaker for the 2 o'clock and the 4 o'clock units, weaker still for the 1 o'clock and the 5 o'clock units, and zero for the other units. The cluster of lines at the center of Figure $7 \mathrm{a}$ concisely represents this pattern of responses at a single position. Since only 5 of the 12 units exhibit positive activity, only five clock hands are displayed. The hour to which a hand is pointing codes the orientation and direction preference of a unit, and the length of the hand codes response strength.

Figure 8a uses the representation described in Figure 7a to combine the activity patterns of all oriented Level 3 units (Figure 6) into a single representation. Each of the $16 \times 16$ locations in this representation depicts the activity of 12 units which span the full range of orientation and direction preferences.

Figure $8 b$ depicts a representation of horizontally oriented complex cells in Level 4 . These cells respond to the sum of output signals from horizontally oriented simple cells of opposite direction of contrast in Level 3, namely from Figures $6 \mathrm{a}$ and $6 \mathrm{f}$. That is why Level 4 cells do not have a black hemidisk in their representation.

Figure $8 \mathrm{c}$ represents the total activity pattern of all Level 4 cells using a variant of the representation used in Figure 8a. In Figure 8c, the activity at a location is coded by a line centered at it. The activity magnitude is proportional to line length, and the orientation is coded by line orientation. Such a representation was called an orientation field by Grossberg and Mingolla (1985a, 1985b).

Figure 8d represents the activity pattern of Level 5 units, which compute a total BC output signal from each BC System position. Each output signal is the sum of thresholded activities from all the orientations computed at that position in Level 4. The spatial distribution of the active urits traces the $\mathrm{Z}$-shaped stimulus contour. The assymetrical representation of the two corners derives from the asymmetrical reaction of the on-field to these corners. A more symmetrical activity profile would have been obtained if the off-unit activity had been taken into account. In particular, the weakening of boundary activity at the upper corner of Figure 8d corresponds to the weakening of concentric on-unit activity at the corresponding location in Figure 5a. In contrast, this is a site of strong offunit activity in Figure $5 b$.

Finally, Figure 9 presents the activity pattern in the field of Level 6 units. The BC signal pattern from Level 5 divides the field into two compartments, or interaction domains: the lower left region (yang) and the upper right region (yin). Inputs from Level 2 trigger a spreading, or filling-in, of activity within each domain, but there is little communication between domains. The final activity level for points within a domain is roughly proportional 


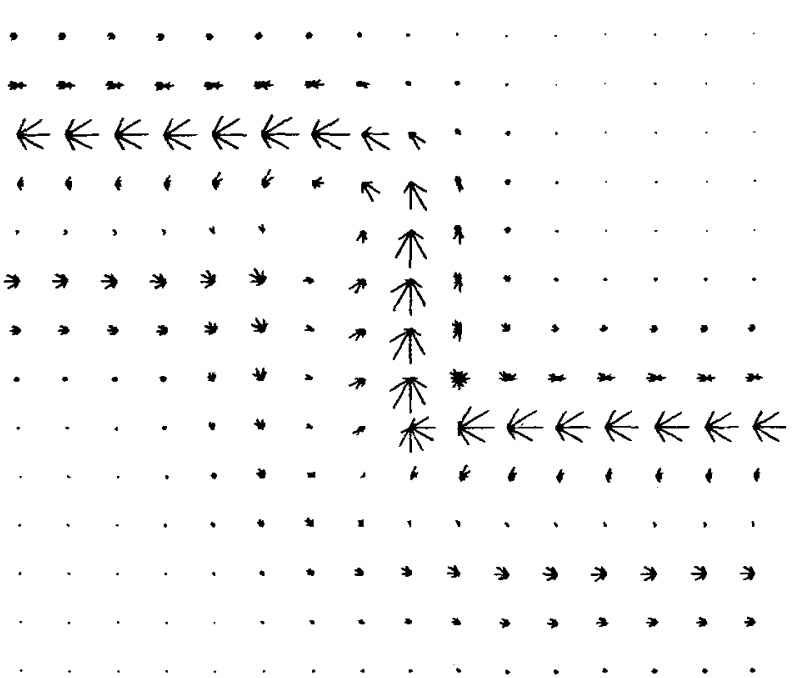

(a)

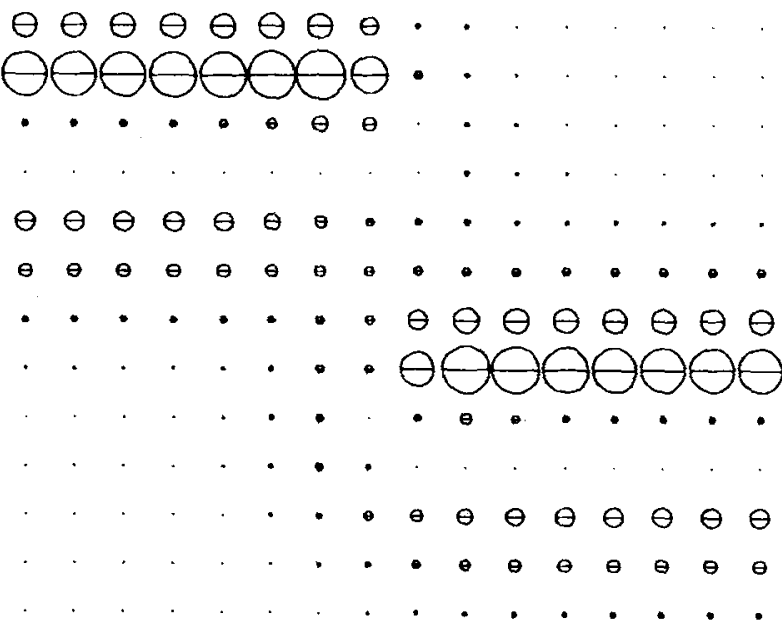

(b)

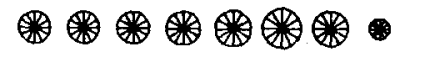

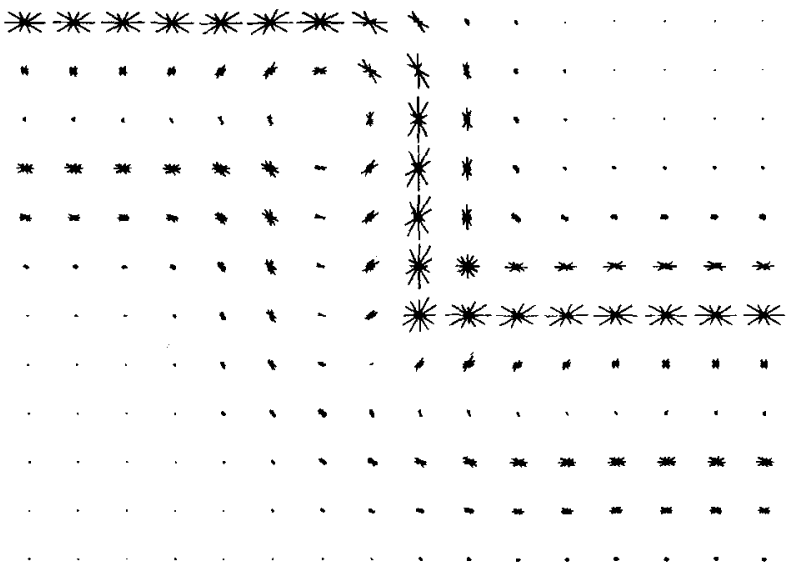

(c)

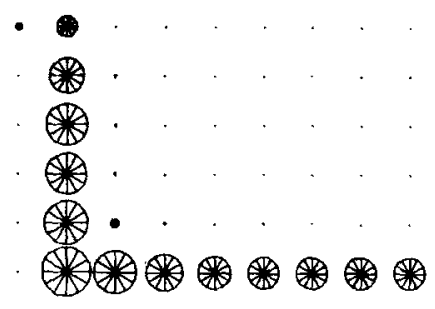

(d)

Figure 8. (a) The combined activity profile of all Level 3 units. Each cluster of lines represents the activity level of 12 units that have the same location. The representational code is described in Figure 7. (b) The activity profile of horizontally oriented direction-of-contrastinsensitive Level 4 units. Fach unit sums the activity of two Level 3 units with the same location and orientation but opposite direction sensitivity. (c) The combined activity profile of all Level 4 units. Each cluster of lines represents the activity level of six units that have the same location. Activity magnitude is coded by line length, and orientation preference is coded by line orientation. (d) Level 5: Output of the Boundary Contour System. Each unit sums the thresholded signal of 6 Level 4 units with the same location. The activity profile traces the shared boundary of the two regions of the yin-yang square.

to the average level of input stimulation due to the corresponding Level 2 region. Because of the increased level of on-units activity on the yin side of the Z-shaped contour, and the decreased level on the yang side, the average activity is smaller within the yang region than in the yin region. The final consequence is that, in Figure 9, the activity pattern of the Level 6 syncytium is qualitatively very similar to that of Figure 4 , the image stimu- lus distribution, except for modest brightness enhancement and attenuation at the Mach corners of the percept. Since the Level 6 activity profile in our model is the counterpart of the brightness percept, the prediction from the simulation is that the percept is close to being veridical.

There are two particularly noteworthy aspects of our introductory example. First, the two portions of the stimulus distributions that have homogeneous luminance levels 


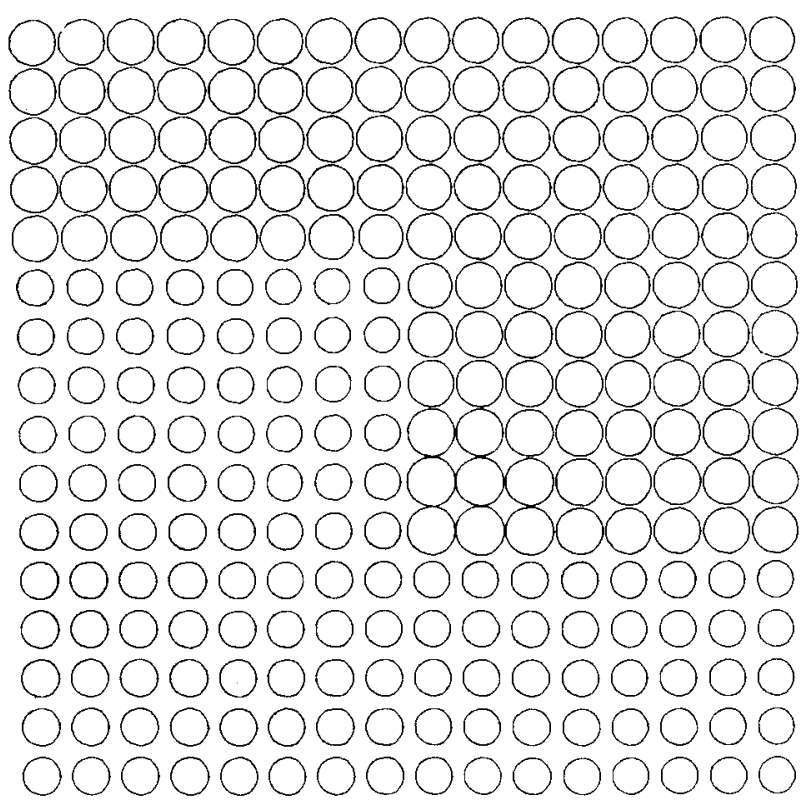

Figure 9. The final filled-in activity profile at Level 6, the syncytium. It contains two homogeneous regions that have different activity levels. This distribution closely matches the stimulus distribution (Figure 4). The Level 6 activity profile predicts the brightness appearance of the stimulus. In this case, the percept is nearly veridical.

correspond to approximately homogeneous portions in the brightness distribution. This is not a trivial result, because vision models that concentrate on edge processing generally fail to make this or any prediction about the appearance of the portions of the image located between the contours.

Second, the model correctly predicts that the more luminant portion of the stimulus (Figure 3 ) will be perceived as the brighter one. However, there are many examples of visual situations in which there is a mismatch between the luminance distribution and the brightness distribution. In particular, two portions of the image may have the same luminance but appear unequally bright, or vice versa. An adequate model of human spatial brightness perception must be able to correctly predict these discrepancies. In the next two sections, we show how the same set of mechanisms handles, in a unified way, both cases of veridical perception and striking brightness illusions. In all of these examples, the output of the model is in close agreement with the perceived brightness distribution.

\section{PART 4 \\ 1-D SIMULATIONS}

All graphical depictions of the 1-D simulations contain four distributions: the stimulus luminance distribution (Level 1), the on-unit distribution (Level 2), the output of the BC system (Level 5), and the syncytium distribution (Level 6), which corresponds to the predicted bright- ness distribution. Cohen and Grossberg (1984) presented their simulations of various brightness phenomena in a similar format. The graphs of the four distributions were scaled separately; that is, each was normalized with respect to its own maximum.

\section{Equally Illuminated Stimulus Patches}

We begin with the simulation of a simple visual situation whose purpose is to set the context for the following simulations. The Level 1 luminance distribution, labeled Stimulus, is presented in the bottom graph of Figure 10a. It portrays the horizontal cross-section of an evenly illuminated scene containing two equally luminant homogeneous patches on a less luminant homogeneous background. The Level 2 reaction of the on-units to such a stimulation, labeled Feature, illustrates the cusp-shaped profiles that correspond to luminance discontinuities. The four boundary contours formed at Level 5 of the system are labeled Boundary. Finally, the top graph, labeled Output, presents the Level 6 filled-in activity profile that embodies the prediction of a brightness distribution qualitatively isomorphic with the luminance distribution. This percept contains two homogeneous, equally bright patches on a darker homogeneous background.

\section{Unequally Illluminated Stimulus Patches: \\ Brightness Constancy}

What happens when the two-patch scene is unevenly illuminated? Figure $10 \mathrm{~b}$ presents a luminance distribution that mimics the effect of a light source off to the right side of the scene. The luminance profile is now tilted, and the right patch has more average luminance than the left patch. One of the classical observations in perceptual psychology is that in such situations the brightness percept does not agree with the luminance distribution. Instead, brightness constancy prevails, indicating that the brightness percept is not determined by surface illumination but correlates with surface reflectance, a physical attribute independent of illumination.

Inspection of the output reveals that our model exhibits brightness constancy. It predicts a percept whose structure is very similar to the preceding, evenly illuminated scene. One factor that contributes to this outcome is the ratio-processing characteristic of the Level 2 on-units. Although the absolute luminance values in the stimulus distributions in Figures $10 \mathrm{a}$ and $10 \mathrm{~b}$ are different, the ratio of the lower to the higher luminance across all edges in both distributions is $1: 3$. Therefore, the activity profiles of Level 2 on-units are very similar in both cases, as is the activity in all subsequent processing stages. The consequence is that the illuminant is effectively discounted.

The importance of luminance ratios for brightness perception was stressed by Wallach $(1948,1976)$. He found that if one region was completely surrounded by another, the brightness of the inner region was predominantly influenced by the size of the ratio of its luminance to the luminance of the surrounding region. Our model provides 


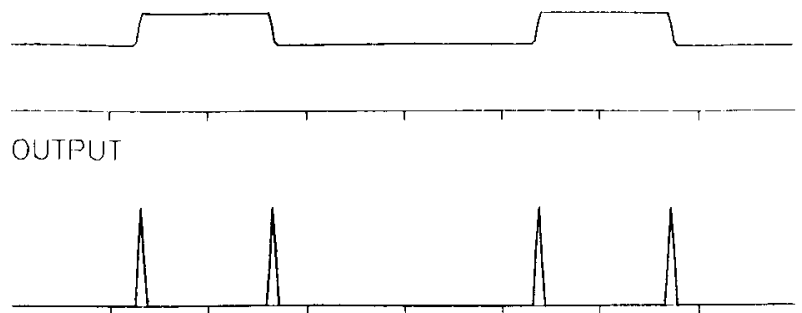

BOUNDARY
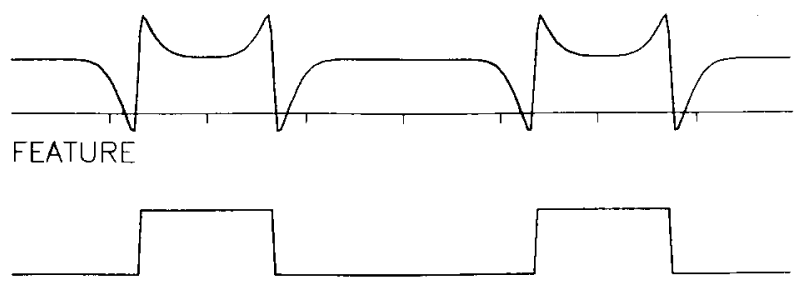

STIMUL.US

(a)

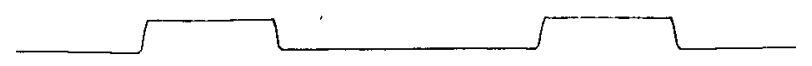

OUTPUT

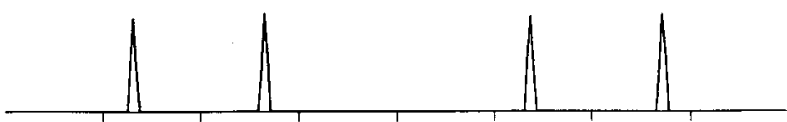

BOUNDARY

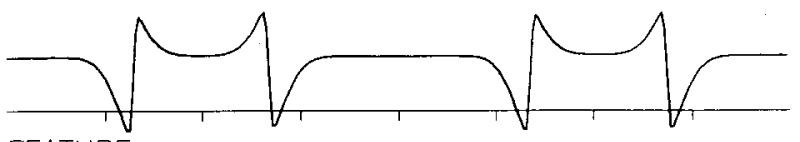

FEATURE

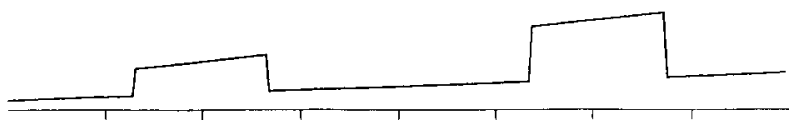

STIMULUS

(b)

Figure 10. One-dimensional simulations of the same scene evenly and unevenly illuminated. In these and all following 1-D simulations, the four graphs, from bottom to top respectively, refer to the Level 1 stimulus distribution (labeled Stimulus), the Level 2 on-cell distribution (labeled Feature), the Level 5 Boundary Contour output (abeled Boundary), and the Level 6 filled-in syncytium (labeled Output). The parameters used in the simulations are listed in the Appendix. Although the two stimulus distributions in Figures 10a and $10 \mathrm{~b}$ are different, the final output distributions are very similar. Thus the model exhibits brightness constancy.

a mechanical explanation of why the ratio principle is effective in such situations. In addition, as will be shown below, the model is applicable to more general visual situations in which multiple regions have multiple neigh- bors, and it provides perceptually correct predictions in situations in which the ratio principle fails.

\section{Brightness Contrast: \\ Narrow Patch and Wide Patch}

Land (Land, 1977, 1986; Land \& McCann, 1971) devised the influential retinex model of color perception, which includes an algorithm for discounting the illuminant. Todorović (1983) and Shapley (1986) have noted that this model cannot account for another classical perceptual effect, the phenomenon of simultaneous brightness contrast. The luminance profile characterizing the favorite textbook example of this phenomenon is depicted in Figure 11a. The luminance distribution is similar to that of Figure 10a in that it contains two patches of medium luminance level. However, the left patch is positioned on a lower luminant background, and the right patch on a higher luminant one. The perceptual consequence is that, despite equal luminance, the two patches look different; the patch on the dark background looks brighter than the patch on the bright background. Inspection of the output in Figure 11a reveals that this is also the prediction of the model.

The reason why Land's model cannot account for this effect is, in part, that it is essentially geared to recover surface reflectance. However, in the phenomenon of brightness contrast, brightness constancy is violated, and two surfaces with the same reflectance look unequally bright. In Land's model, the relative brightness of two regions is essentially determined by the product of ratios of luminances of locations situated along paths between the two regions. Shapley (1986) has shown that for two homogeneous regions with the same reflectance that are contained within an evenly illuminated scene composed of homogeneous regions, this ratio is 1 . Consequently, according to the Land model, such regions should have the same brightness. However, the phenomenon of brightness contrast shows that this is not necessarily the case.

In the response profile of the on-units, labeled Feature in Figure 11a, the interior of the left patch contains a higher level of activity than the interior of the right patch. On the basis of a graph similar to this figure, Cornsweet (1970, p. 352) concluded that a Fourier analysis approach was able to account for brightness contrast. Such an explanation could be interpreted by using the same lateral inhibitory mechanisms that are involved in generating Mach bands. In contrast, we suggest that brightness contrast depends essentially on the filling-in process (see Fry, 1948). To illustrate the role of filling-in, consider Figure $11 \mathrm{~b}$. The luminance distribution in Figure $11 \mathrm{~b}$ is similar to the one in Figure 11a, but the gray patches are larger. Consequently, the central portions of the on-unit profiles that correspond to the stimulus patches in Figure $11 \mathrm{~b}$ have the same activity magnitude. Hence, these activity profiles cannot account for the difference in appearance. However, the filled-in activity patterns within each region of the Level 6 output in Figure 11b are different and homogeneous. This result accords with 


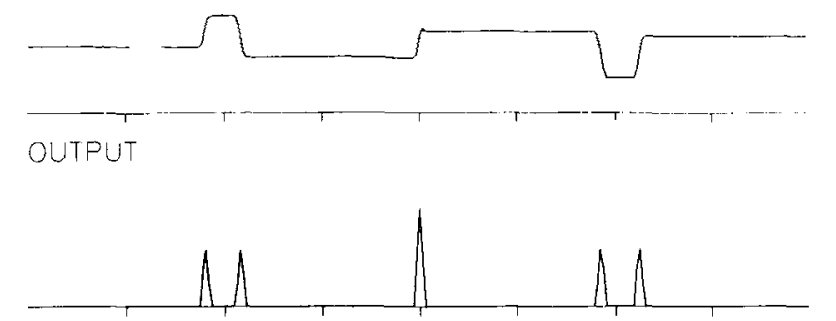

BOUNDARY
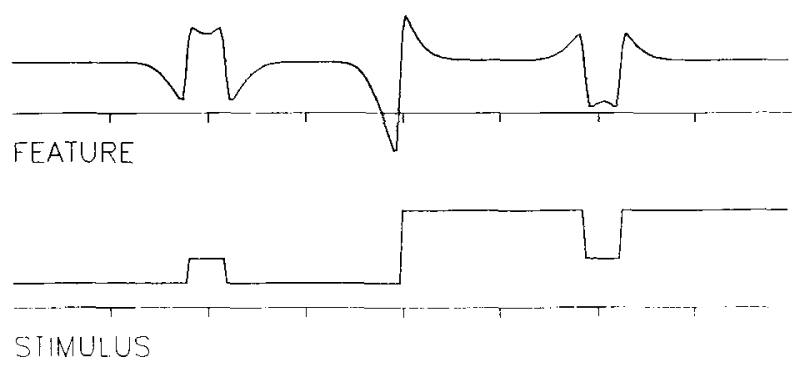

(a)

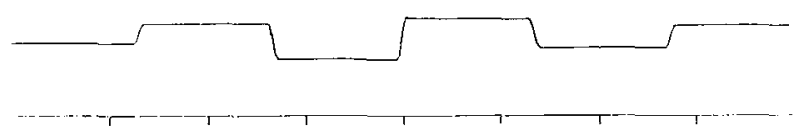

OUTPUT

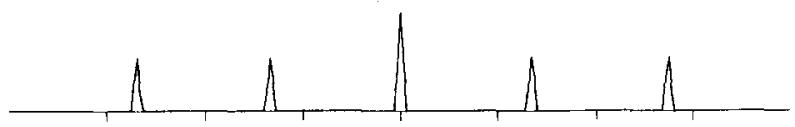

BOUNDARY
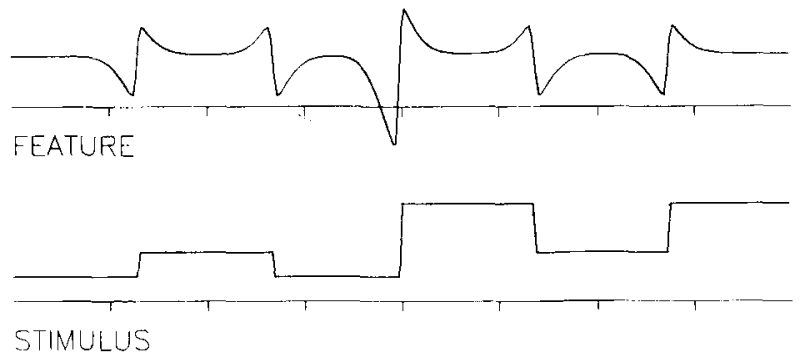

(b)

Figure 11. Simultaneous brightness contrast. The stimulus contains two medium luminance patches, the left one on a low-luminance background and the right one on a high-luminance background. In Figure 11a, the two patches are narrow; in Figure 11b, they are wide. In both cases, the model output predicts the left patch to look brighter than the right patch. In contrast, in the on-unit profiles, the centered activity levels corresponding to the two patches are different in Figure 11a but not in Figure 11b. These simulations demonstrate that brightness contrast cannot be explained solely by contour generated activity, but that a filling-in process is also necessary.

the study of Yund and Armington (1975), who reported that although the strength of the simultaneous brightness contrast effect is smaller for larger test regions, it persists for test regions up to $10^{\circ}$. This decrease of the ef- fect with region size cannot be explained by the ratio principle, since it is insensitive to region size, but is predicted by our model. Due to diffusion, the brightness of a region correlates with the average amount of corresponding Level 2 activity. For a gray patch on a dark surround, this activity consists of the overshoots, corresponding to the region's edges, and the "valley" between them. With increasing region size, the relative proportion of the valley also increases (compare the portions of the Level 2 profiles that correspond to the left patches in Figures 11 a and $11 \mathrm{~b}$ ). In consequence, the mean Level 2 activity that corresponds to the gray patch decreases. An analogous analysis shows that for gray patches on bright surrounds, the larger patch is predicted to look brighter than the smaller patch.

A more subtle quantitative difference between Figures $11 \mathrm{a}$ and $11 \mathrm{~b}$ is also worth noting. In Figure 11a, the narrow luminance patches interact with the model parameters to cause filled-in brightness levels such that the middle gray patch on the left is brighter than the white background on the right, and the middle gray patch on the right is darker than the dark gray or black background on the left. In Figure $11 b$, the wider luminance steps cause filled-in brightness levels such that the reverse inequalities obtain, as is also the case in vivo. The reason for this reversal is, in part, that the boundary cusps (overshoots) in the FC System reactions to the patches form a smaller relative part of each patch in Figure 11b than in Figure 11a. Other parameters that influence this reversal are the model's baseline activity level and the relative amount of FC System contrast enhancement at image edges. To the extent that an FC System pattern such as that shown in Figure 11b always occurs in vivo, the explanation of brightness contrast in response to images of this type depends essentially upon filling-in.

On the other hand, informal observations suggest that, depending on the particular luminance levels and region areas, either set of brightness relationships may be perceptually realized. The characterization of the parameters that lead to one or the other set of relationships requires further psychophysical investigation.

Finally, in these simulations, the two portions of the background, to the left and to the right of the gray patch, are unequally bright. This is especially noticeable in the case of the dark background in Figure 11b. This outcome is an artifact of the one-dimensionality of the simulation, in which the two parts of the background are isolated from each other by the gray patch. In two dimensions, the background surrounding the patch is a topologically connected region, and the diffusional processes can freely act to homogenize it throughout.

\section{Varieties of Brightness Contrast: \\ Graded Backgrounds and Contrast Constancy}

Shapley (1986) presented a new variant of brightness contrast, which our model also explains. This luminance distribution is presented in Figure 12a. The two equiluminant patches from the classical version of the ef- 


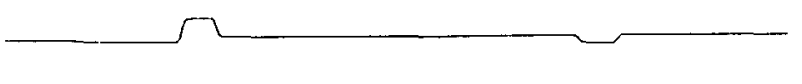

OUTPUT

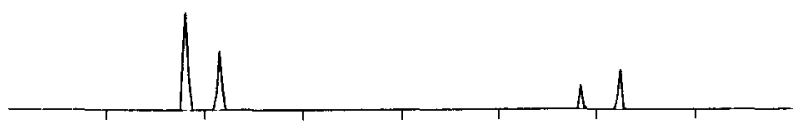

BOUNDARY

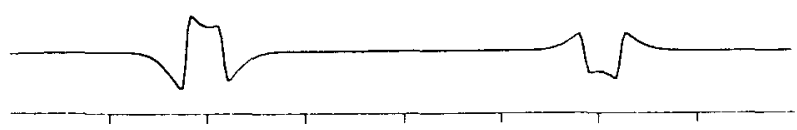

FEATURE

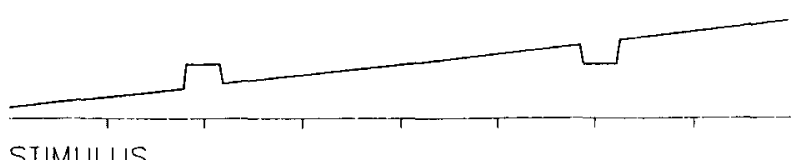

(a)

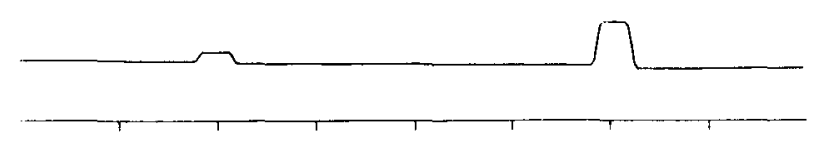

OUTPUT

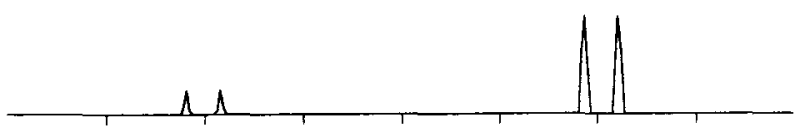

BOUNDARY

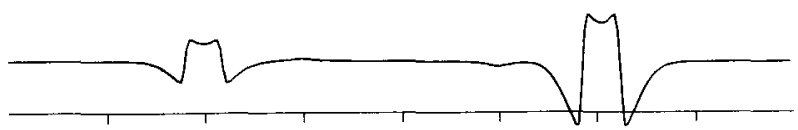

FEATURE

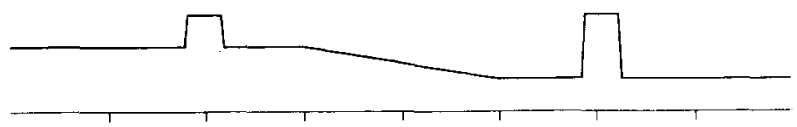

STIMULUS

(c)

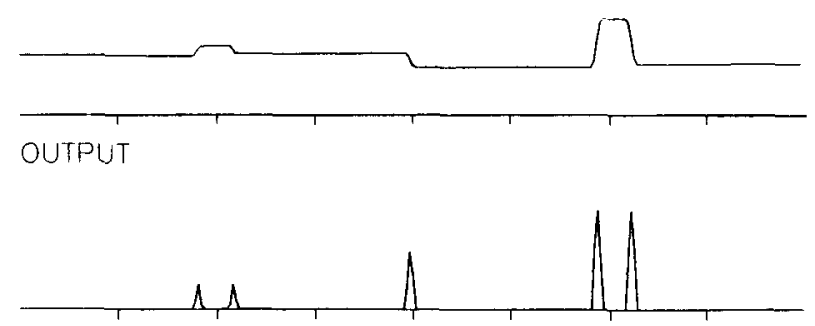

BOUNDARY

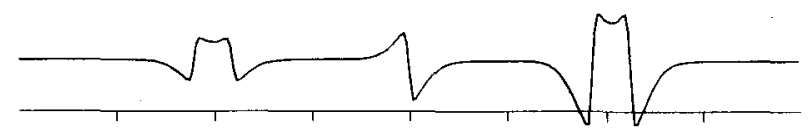

FEATURE

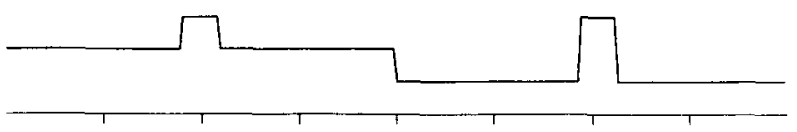

STIMULUS

(b)

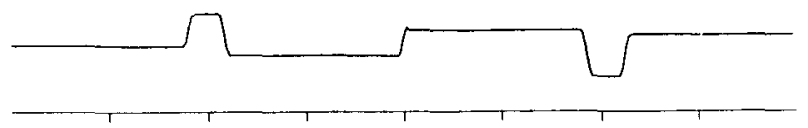

OUTPUT

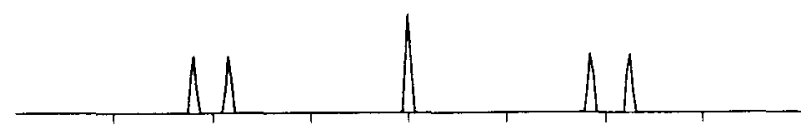

BOUNDARY
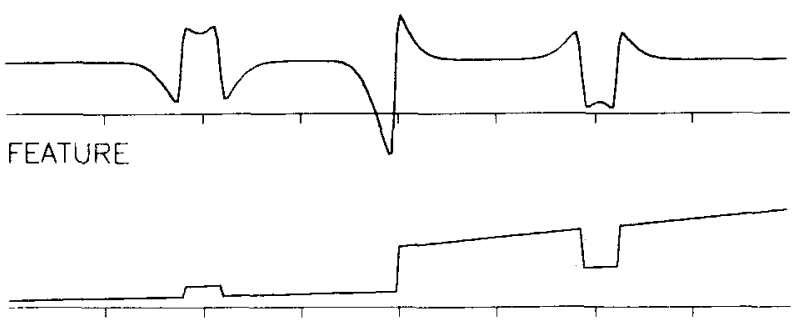

STIMULUS

(d)

Figure 12. Varieties of brightness contrast involving non-classical background conditions. (a) The gradient background. (b) Both patches are more luminant than their backgrounds. (c) The same as (b), but with a gradient transition between the backgrounds. (d) Unevenly illuminated standard brightness contrast stimulus. "Contrast constancy" is predicted.

fect are retained, but the background is different. Instead of two regions of homogeneous and very different luminance, as in Figure 1la, the background now consists of a continuous gradient of luminance sloping from a high value on the right of the image to a low one on the left. The perceptual effect is similar to the classical phenomenon: the left patch looks brighter than the right one. Furthermore, the gradient itself is not very prominent in the percept. These effects are also predicted by the model output.
Shapley $(1986$, p. 47) pointed out a common aspect of the classical and the new versions of the effect. In both cases, the luminance ratio across the borders of the two patches are of opposite sign, and he suggested an explanation based upon this sign difference. However, it is not only the sign, but also the size of the luminance ratio that influences brightness. Figure $12 \mathrm{~b}$ is a luminance profile that was studied by Arend, Buehler, and Lockhead (1971). The visual situation is similar to the classical contrast profile of Figure 11a, except that now both equiluminant 
patches are more luminant than their backgrounds. Thus, the sign of the luminance ratio is the same. Nevertheless, Arend et al. found, and our model output predicts, that the patch with the larger ratio is perceived as the brighter one. Arend et al. also studied a version of the effect similar to the Shapley (1986) gradient version. This luminance distribution is presented in Figure 12c. Again, the perceptual effect is consistent with the output of our model in Figure 12c.

The phenomenon simulated in Figure $12 \mathrm{c}$ provides a useful antidote to theoretical concepts that do not sufficiently take into account effects of image context on brightness percepts. In particular, equally luminant patches are mapped into non-equally bright percepts, while a background composed of non-equally luminant regions is mapped into approximately equally bright regions. Thus, the context-sensitive mapping from luminance to brightness can convert identities into differences and differences into identities.

A related version of these effects is presented in Figure 12d. It portrays an unevenly illuminated brightness contrast situation. Note that the right patch is now more luminant than the left one. However, our model predicts that the percept will be similar to classical brightness contrast. In other words, we predict an effect of "contrast constancy." Such a visual situation has, to ourknowledge, not been studied yet.

A comparison of Figures 12a, 12c, and 12d calls attention to an issue concerning the choice of model parameters, and to possible influences of different parameter choices across the multiple spatial scales of a more complete model. Within these figures, the sloping background luminance gradients are converted into approximately uniformly bright percepts. This is partly due to the relative insensitivity of the FC System to linear luminance gradients. It is also due, however, to the size of the threshold in the BC System filters relative to the size of the cusps near the patch edges in the FC System activity patterns. Had these thresholds been chosen smaller, then more BC System boundaries would have been activated in the FC System cusp regions and the percept of background brightness would have been more nonuniform in these regions. Once this is realized, it also becomes clear that any mechanism that enhances cusp sizes or, more generally, generates a spatially nonuniform FC System activity pattern can generate a more dense spatial distribution of boundaries, or boundary web (Grossberg, 1987a; Grossberg \& Mingolla, 1987). Such a boundary web can trap local FC System contrasts into small boundary compartments, and can thereby convert a nonuniform luminance pattern into a nonuniform brightness percept. In addition, the parameters within one spatial scale may not generate a boundary web in response to a particular nonuniform luminance pattern, whereas the parameters within a different spatial scale may generate such a boundary web. Then the total brightness percept (at a given perceived depth) would be a weighted sum of uniform and nonuniform spatial patterns.

\section{Brightness Assimilation}

Shapley and Reid (1986) have studied a more complex visual situation whose luminance profile is depicted in Figure 13. This profile can be derived from the standard brightness contrast profile (Figure $11 \mathrm{~b}$ ) by the introduction of two additional test regions. One of these regions is centered and wholly contained within one of the two

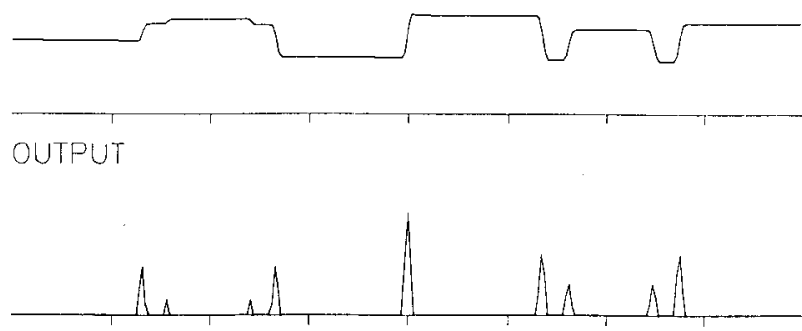

BOUNDARY

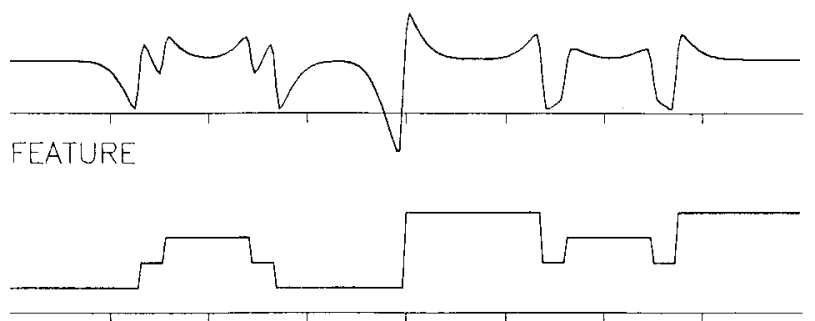

STIMULUS

(a)

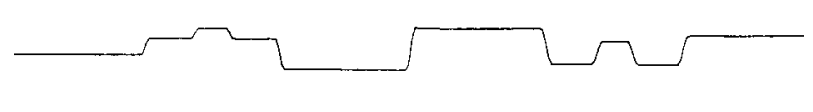

OUTPUT

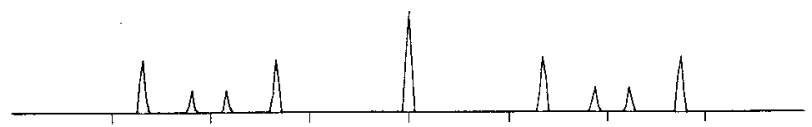

BOUNDARY
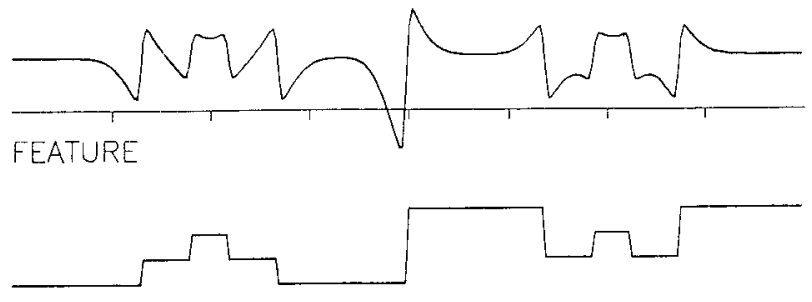

STIMULUS

(b)

Figure 13. The Shapley and Reid (1980) assimilation stimulus. The stimulus distribution is the same as in Figure 11b, with the addition of two equiluminant test regions centered on the two gray patches. The model output correctly predicts that the left test region looks brighter than the right test region: (a) wide test regions; (b) narrow test regions. 
original equiluminant gray patches; the other is centered and contained within the other gray patch. The test regions have the same luminance level, which is higher than the luminance level of surrounding gray patches. However, the experiment showed that the left test region looks brighter than the right one. A gradient version of this distribution showed similar results.

Shapley (1986) and Shapley and Reid (1986) claimed that this effect could not be due to brightness contrast, and that it was, instead, an instance of another classical brightness effect, the phenomenon of brightness assimilation (Helson, 1963). They pointed out that the ratio of the luminance of each of the innermost patches to the luminance of the immediately surrounding region was the same. If classical brightness contrast were exclusively due to the luminance ratio, then a new explanatory principle would be needed to explain the finding. Wallach (1976) also found that, in a series of three nested regions, the ratio principle was violated.

Inspection of the output in Figure 13 reveals that our model correctly predicts the difference in brightness between two inner regions. Thus, the model accounts for Wallach's ratio principle as well as its violation in more complex situations. In particular, the processing by oncell units can lead to either a contrastive or an assimilative brightness effect. The outcome depends upon the total configuration of FC signals that induce the filling-in within the compartments defined by the $\mathrm{BC}$ signals.

In particular, two aspects of the model contribute to the brightness assimilation effect described by Shapley and Reid (1986), one at Level 2 and the other at Level 6. The first is the context-sensitive response of the Level 2 oncells to two or more contiguous luminance steps. The amplitudes of the overshoots and the undershoots, and the exact course of the Level 2 profile corresponding to a luminance step, are influenced by the presence and polarity of nearby luminance steps. In this way, the two backgrounds can differentially affect the test regions even across the surrounding gray patches. In particular, the Level 2 profile corresponding to the right test region in Figure 13a is depressed relative to the left test region. As reflected in Shapley and Reid's data, this effect of the background should decrease with the size of the width of the surrounding gray patches.

A second way in which nearby regions can influence each other occurs at the diffusion stage. Although the presence of a boundary between two regions strongly attenuates the interaction between them, it may not annihilate it completely if the strength of $\mathrm{BC}$ signals can vary significantly with the amount of contrast and the spatial scale of the FC patterns, as it does in Figure 13b. If a weak boundary separates two regions with different FC activity levels, then activity from each region will, to a certain extent, diffuse across the boundary into the other region. This process will tend to increase the final filledin activity level in the left test region of Figure 13b and to reduce it in the right test region. This is because the left test region is surrounded by a region (corresponding to the left gray patch) whose Level 2 activity profile is, on the average, larger than the average strength of the Level 2 profile of the region (corresponding to the right gray patch) surrounding the right test region. Thus, due to the combined effect of the small size of the test patches and the large size of the gray patches, the left test path appears brighter than the right one even though their FC patterns are similar.

A third factor is the possible influence of multiple spatial scales (Grossberg, 1987b). A small test region may generate boundary signals in one scale but not in another. Featural filling-in within the latter scale will therefore cross the perceptual locations subtended by the small test region. If that region is surrounded by a darker patch, the total filled-in brightness percept, assuming that it is the weighted sum of the filled-in activity levels across all scales within that region, will tend to be darker.

\section{The Craik-O'Brien-Cornsweet and Brightness Bull's-Eye Effects}

One of the most attractive brightness phenomena is the Craik-O'Brien-Cornsweet effect, or COCE (Cornsweet, 1970; see Todorović, 1987, for a review). One version of the COCE is presented in Plate 1. Readers unfamiliar with this effect might suppose that since the left rectangle is brighter than the right rectangle, it is also the more luminant one. However, the luminance of the two rectangles is actually identical, except for a luminance cusp overshoot at the left flank and a luminance cusp undershoot at the right flank of the midline. The illusory nature of the phenomenon is most easily demonstrated by the oc-

Plate 1 (opposite). The Craik-O'Brien-Cornsweet effect (COCE). The luminance distribution representing this display is shown in Figure 14a. The left rectangle looks brighter than the right rectangle although they have identical luminance, except for the cuspshaped profile of their shared vertical border. (From Todorović, 1987.)

Plate 2 (opposite). The 2-D cusp distribution without the bounding contour. This display differs from Plate 1 only with respect to the background. The dark background in Plate 1 has been replaced with a background whose luminance is equal to the average luminance of the two central rectangles. There is no illusory brightness effect in this display comparable to the COCE in Plate 1 (From Todorovic, 1983, 1987.)

Plate 3 (p. 259). The impossible brightness staircase. The stimulus distribution corresponding to this display is presented in Figure 20a. The display consists of four $L$-shaped regions whose shared borders have a cusp-shaped luminance profile. When this display is occluded such that only portions of two neighboring regions are visible (see Figure 21a for an example of the resulting stimulus distribution), the result is a standard case of the COCE. If the occlusion demonstration is carried out for all four neighboring region pairs, the first member of the pair in the clockwise direction always appears brighter than the second member. In the unoccluded display, no stable pattern of brightness relationships between neighboring regions emerges. (From Todorović, 1983.) 


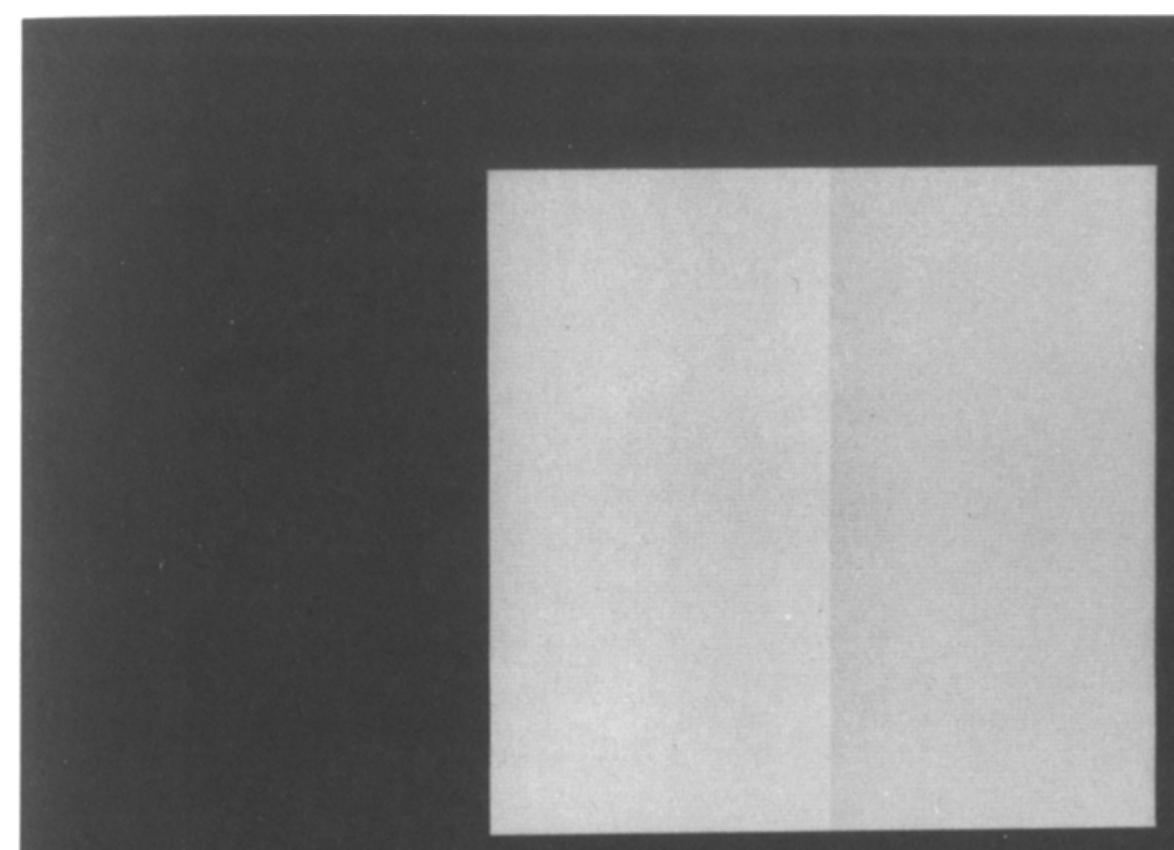

Plate 1

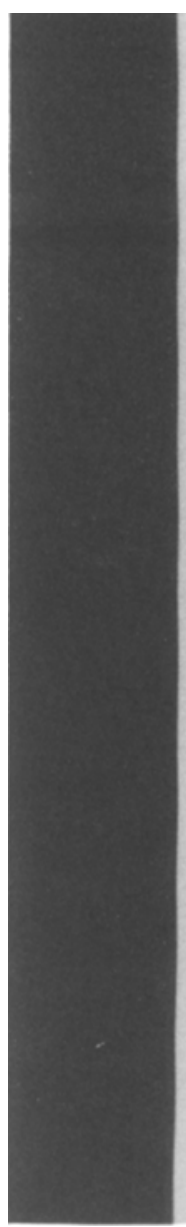

Plate 2 


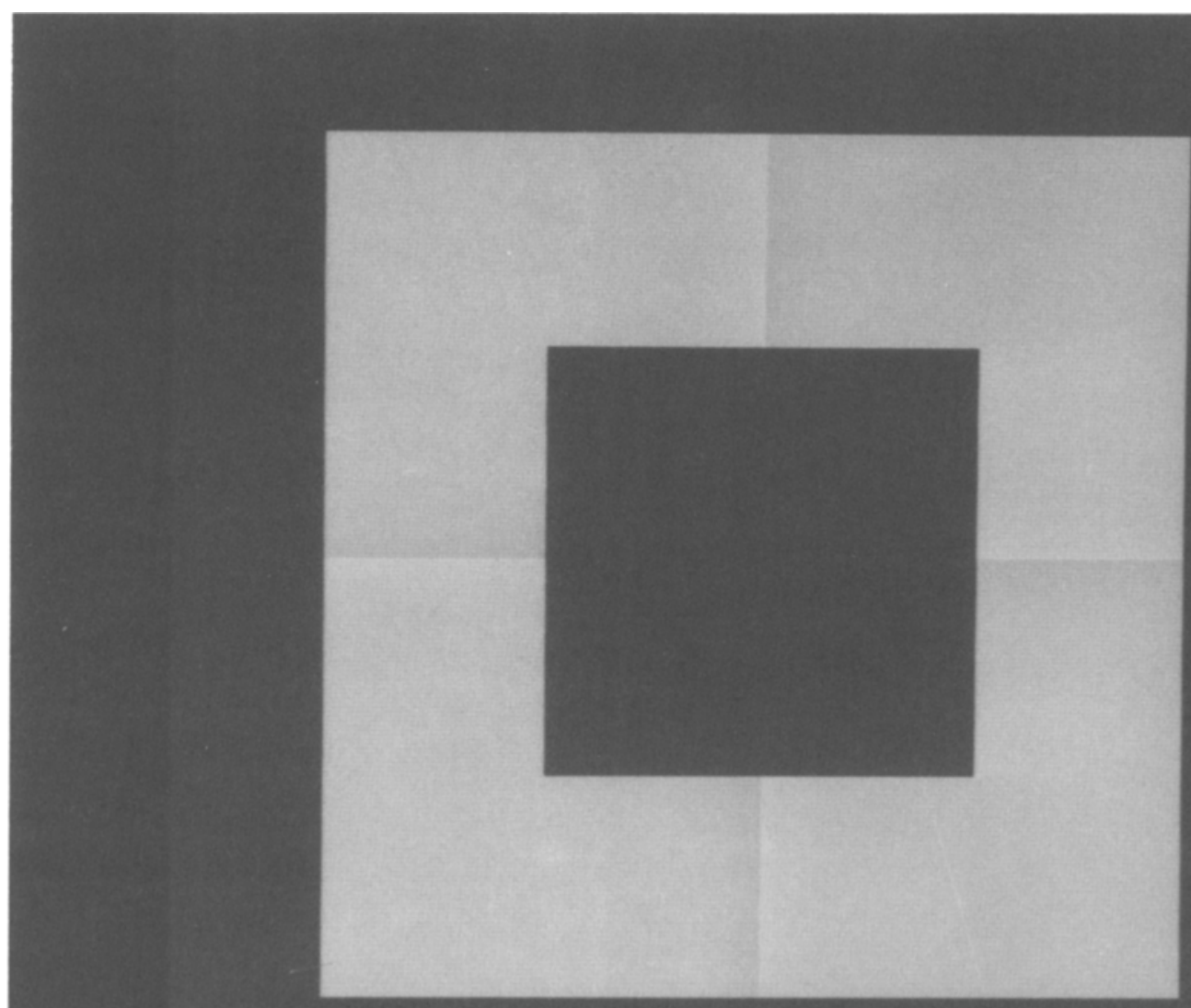

Plate 3 


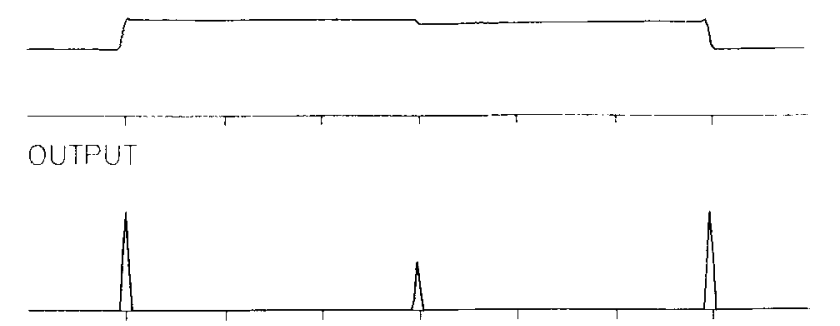

BOUNDARY
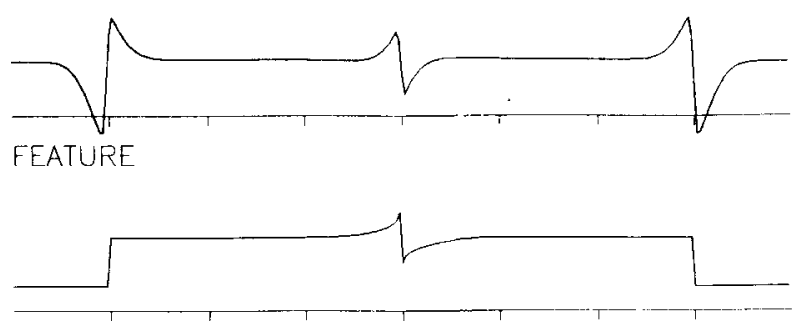

STIMULUS

(a)

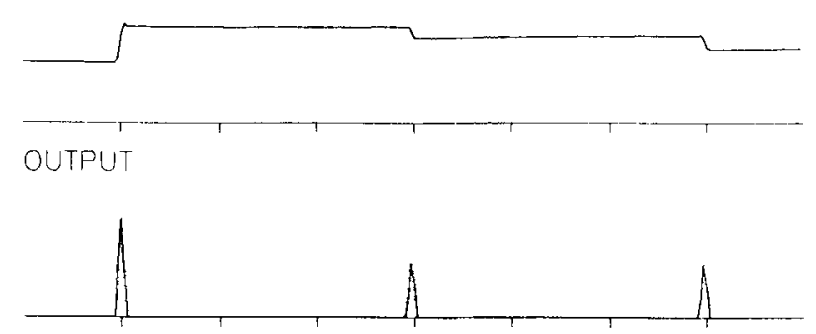

BOUNDARY
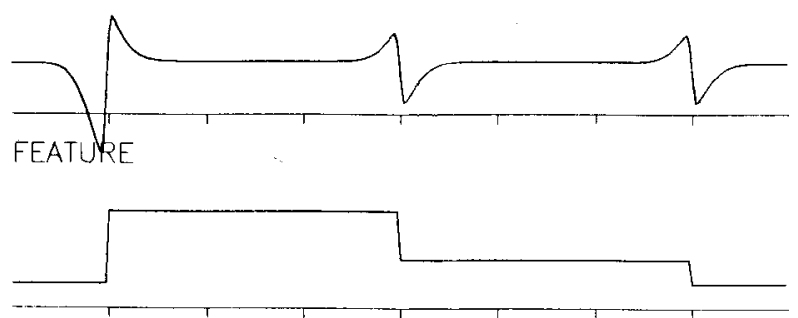

STIMULUS

(b)

Figure 14. The 1-D simulations of the COCE and its nonillusory counterpart. (a) The COCE. (b) A luminance step on a less luminant background. The outputs of both simulations predict a step-shaped brightness profile.

clusion of the contour region. Placing a pencil or a piece of wire vertically across the midline in Plate 1 causes the two rectangles to appear equally bright.

A representation of the 1-D luminance distribution of a horizontal cross-section of Plate 1 is given in the bottom graph, labeled Stimulus, of Figure 14a. Such a profile includes both the luminance cusps and the equally luminous dark background at the left and right side of the stimulus profile. For comparison, the bottom graph in Figure 14b displays the nonillusory counterpart of the cusp distribution. It has the form of a simple luminance step. The cusp distribution and the step distribution are examples of different stimuli causing similar percepts (Ratliff \& Sirovich, 1978). This is also the prediction of our model presented in the top graphs in Figures 14a and $14 \mathrm{~b}$.

The similarity of the perceptual effects of the two images is already apparent in the Level 2 on-cell activity profiles (see Ratliff \& Sirovich, 1978, for some related simulations). The loci of abrupt luminance changes in the stimuli induce extended cusp-shaped profiles in the oncell activity pattern, whereas the regions of homogeneous but different luminance induce similar response levels. Some authors have argued that the similarity of activity patterns at this level, which is also predicted by the Fourier analysis approach, is sufficient to explain the similarity of percepts (Bridgeman, 1983; Cornsweet, 1970; Foster, 1983; Laming, 1983; Ratliff \& Sirovich, 1978). Others have been critical of this idea (Arend, 1973; Arend \& Goldstein, 1987; Davidson \& Whiteside, 1971; Grossberg, 1983; Todorovic, 1983, 1987). The obvious difficulties for such an account are that it does not explain why the left region is perceived to be brighter than the right one, or why locations with different activities within the same region in Level 2 appear to have the same brightness in the final percept.

The model's additional processing stages enable it to explain both the similarity of the percepts and the shapes of their brightness profiles. This is achieved through the model's account of how the BC and FC Systems interact. The output of the BC System (Level 5) is presented in the second graph from the top in Figures $14 \mathrm{a}$ and $14 \mathrm{~b}$. Only the largest local changes in the Level 2 profiles are reflected in the BC output pattern. The interaction of the Level 5 BC output and the Level 2 FC output at the Level 6 syncytium, presented in the top graphs in Figures $14 \mathrm{a}$ and $14 \mathrm{~b}$, predicts the brightness percept. The difference in the activity levels between the left and the right portions, especially in the case of the COCE, is noticeable but small, but so is the perceived brightness difference.

Just as the model can handle multiple steps of different polarity, as in Figures 10-14, it can also handle complex stimuli involving several cusp or sawtooth distributions of different polarities, as shown in Figure 15. Imagine a circularly symmetric 2 -D luminance distribution, whose luminance cross-section along any diameter is given in the bottom graph of Figure 15a. The appearance of the central portion of such a distribution is a brightness bull'seye (Arend, 1973; Arend et al., 1971; Arend \& Goldstein, 1987), as predicted by the top graph, labeled Output, in Figure 15a.

This filled-in bull's-eye percept in Figure 15a is generated when the sawtooth luminance pattern is surrounded by a bright background. If the background is sufficiently dark, as in Figure 15b, the difference in brightness between the outermost and the middle band may disappear, as in Figure 15b. Our informal observations of small 


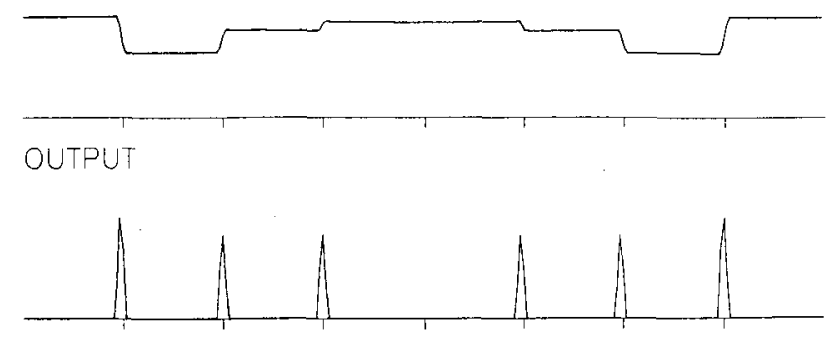

BOUNDARY

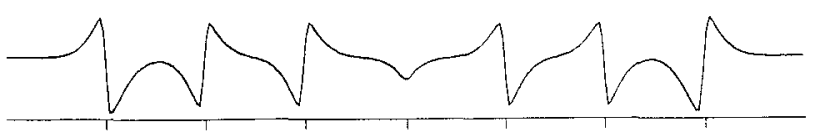

FEATURE

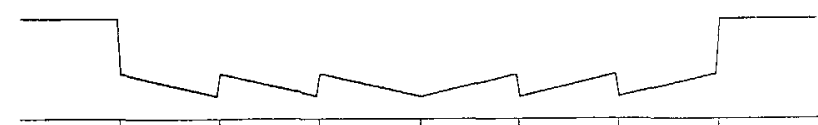

STIMULUS

(a)

OUTPUT

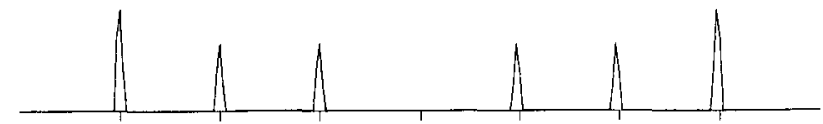

BOUNDARY

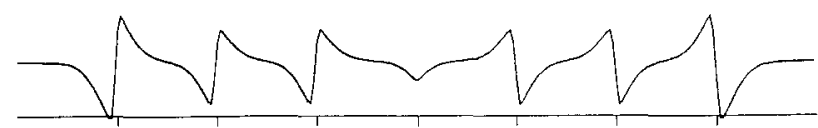

FEATURE

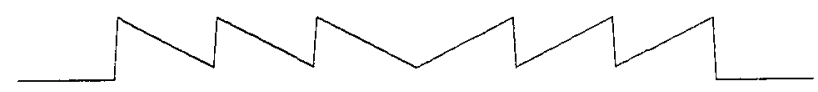

STIMULUS

(b)

Figure 15. The bull's-eye. The luminance distribution is a radial sawtooth (Arend \& Goldstein, 1987) centered on a high-luminance background (a) and on a low-luminance background (b). The graphs of the two luminance distributions are scaled separately. $A$ brightness bull's-eye is generated in the output of (a) but not in the output of (b). See text for details.

bull's-eye patterns on large backgrounds are in the same direction as the model's prediction. A change of the brightness difference between the two outermost luminance bands with the change of the luminance of the surround is to be expected, because the brightness contrast effect is known to decrease with distance (Heinemann, 1972), and thus should more affect the out- ermost band than the middle band. However, Arend (personal communication, 1987) observed no strong effects of the surround luminance on the relative brightness of the bull's-eye bands and, in particular, found that the outer band looked darker than the other bands even on a dark surround. His stimulus involves a relatively large bull'seye on a small surround. In such a configuration, the effect of the surround may be reduced on the bull's-eye percept. An additional complicating factor is the following. Suppose that a 2-D luminance pattern is generated by rotating a 1-D sawtooth pattern. Let the mean luminance of all bands in the 1-D sawtooth be the same, as in Figures $15 \mathrm{a}$ and $15 \mathrm{~b}$. Then the mean luminance of the 2-D bands decreases as a function of their distance from the center. On this ground alone, the outermost bands should look darkest, and thus could more easily counteract effects of the surround. In addition, this percept may be susceptible to effects of multiple scales. Clearly, more parametric experimental data are needed.

\section{Effects of Changing Model Parameters}

In the above simulations, a definite choice of model parameters has necessarily been made (see the Appendix). This section illustrates how related parameter choices influence model behavior.

Figure 16 illustrates the effect of two parameter changes within the model upon the luminance profiles of Figure 14. These changes do not alter the important qualitative properties of the model's filled-in Level 6 profiles. In Figures $16 \mathrm{a}$ and $16 \mathrm{~b}$, the BC signals to Level 6 are rendered more uniform in size by the transformation of the BC patterns in Figures 14a and 14b through a sigmoid, or S-shaped, signal function. This transformation has only a minor effect on the filled-in activity pattern at Level 6 due to the strengthening of the middle $\mathrm{BC}$ signal. In Figures $16 \mathrm{c}$ and $16 \mathrm{~d}$, the parameters of the syncytial diffusion at Level 6 are chosen so that the same BC signals used in Figure 14 have a greater effect on the reduction of diffusion between boundary compartments (see Appendix for details). This manipulation strengthens Mach-band-like effects corresponding to large luminance discontinuities. Both of these parametric variations may well occur across species and individuals in vivo.

Figure 17 depicts another robust parametric property of the Level 2 activity patterns in the model. This is the property, exploited in the previous simulations, whereby Level 2 cells maintain their sensitivity to the reflectances, or relative luminances, near luminance steps as the overall luminance level of a stimulus is parametrically varied. This reflectance-processing property is due to the fact that the Level 2 cells obey membrane, or shunting, equations (see Appendix for details). In Figure 17, each curve corresponds to a constant ratio of the luminances $L_{1}$ and $L_{2}$ on either side of a luminance step. The on-cell activity pattern generated by such a step is a cusp, as in Figure 14. Each curve plots the maximum activity of the cusp generated at a constant ratio, $L_{2} / L_{1}$, as overall luminance is parametrically increased. Each curve increases accord- 


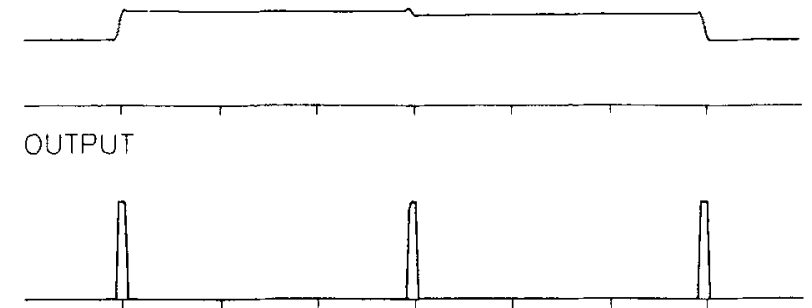

BOUNDARY

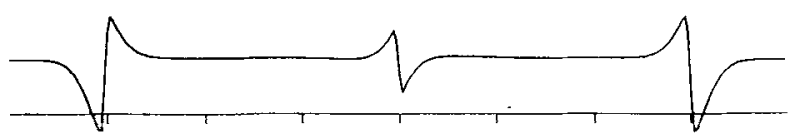

FEATURE

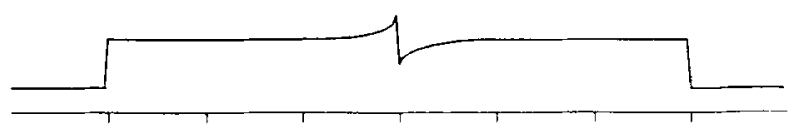

STIMULUS

(a)

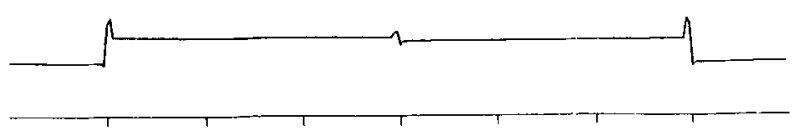

OUTPUT

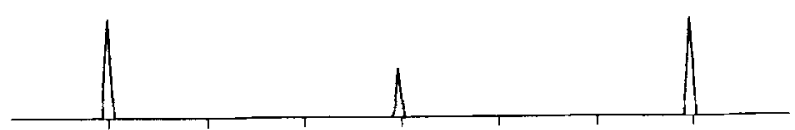

BOUNDARY

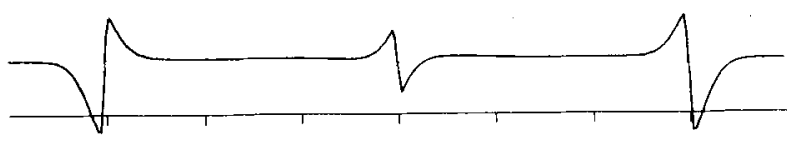

FEATURE

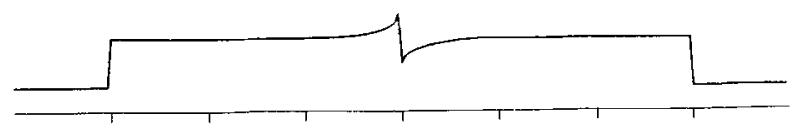

STIMULUS

(c)

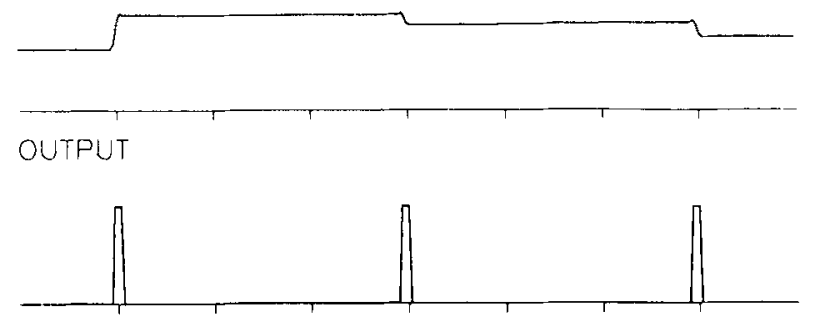

BOUNDARY
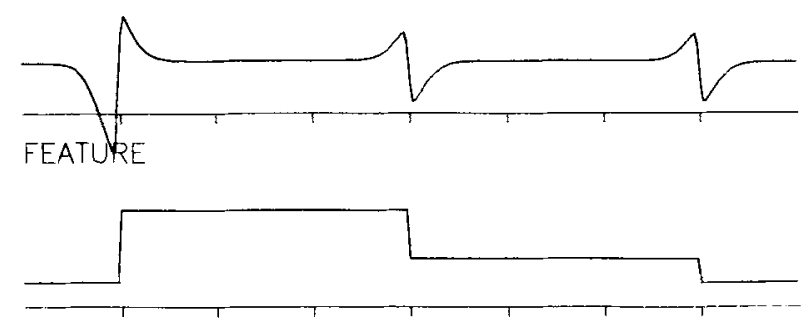

STIMULUS

(b)

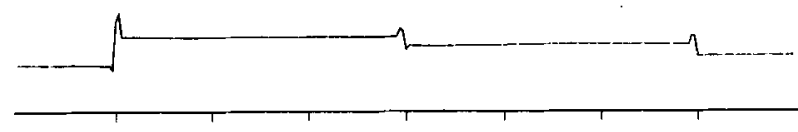

OUTPUT

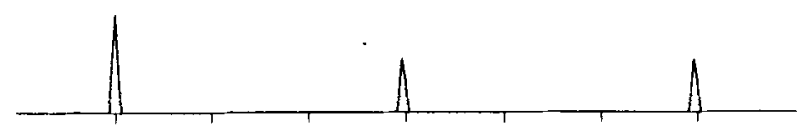

BOUNDARY
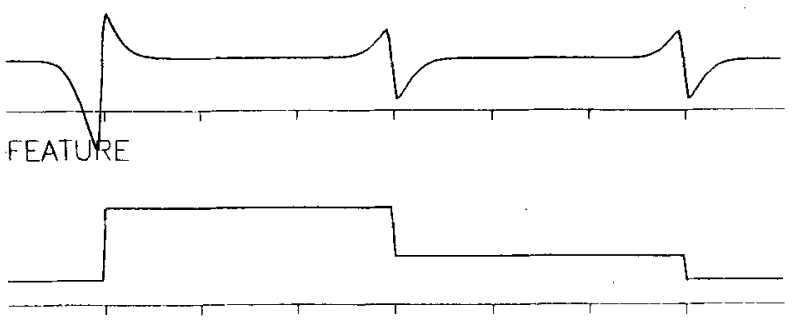

STIMUIUS

(d)

Figure 16. The effects of two parameter changes on simulations in Figure 14. (a, b) Transformation of the Boundary Contour (BC) output through a sigmoid function. (c, d) Increasing the modulation effect of the BC signal on the filling-in process.

ing to a Weber law property until it asymptotes at an activity level that is characteristic of the ratio $L_{2} / L_{1}$ (Grossberg, 1983). Thus, large luminance values do not saturate the on-cell responses. Instead, at large luminances, oncells remain sensitive to input reflectances. The stimulus values used in all simulations fall between the dotted vertical lines, and hence within the luminance range of good ratio processing.

\section{PART 5 \\ THE 2-D SIMULATIONS}

We now present simulations of brightness phenomena using the 2-D implementation of the model. A number of interesting brightness phenomena can be defined and demonstrated only in two dimensions. Arend and Goldstein (1987) have, in particular, used the curl operator 


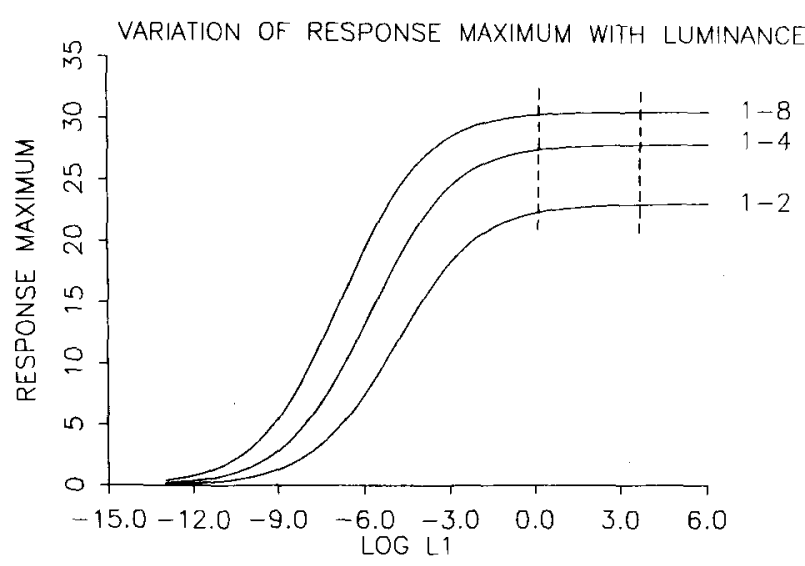

Figure 17. Luminance step processing by on-cells across a range of luminances. Stimulus distributions are luminance steps with three values of the ratio $L_{2} / L_{1}$ of the higher to the lower $\left(L_{1}\right)$ luminance: $2 / 1,4 / 1$, and $8 / 1 . X$-axis: $\log L_{1} . Y$-axis: maximum value of on-cell activity profile. The simulations occur in the luminance range between the vertical dashed lines, and hence exhibit good ratio processing.

of vector calculus to diagnose important properties in 2-D images that are not found in 1-D images. Our results show, however, that the curl is not needed as a model mechanism for the explanation of such brightness phenomena. In all the following simulations, we present Level 1, the stimulus distribution, and Level 6, the filledin activity distribution that predicts the brightness of the percept. In many instances, we also present the intermediate activity distributions from Level 2 and Level 5.

\section{The COCE With and Without}

\section{a Bounding 2-D Region}

We first consider a 2-D manipulation of the COCE that has no 1-D analogue. Plate 2 (from Todorović, 1983, 1987) contains a luminance cusp embedded into a homogeneous luminance field. The only difference between Plates 1 and 2 is in the replacement of the dark bounding background in Plate 1 with a region in Plate 2 whose luminance equals the average luminance of the two central rectangles. The perceptual consequence of this change is an almost complete loss of the COCE: portions of Plate 2 at some distance from the luminance cusp have a similar homogeneous gray appearance. Related displays were studied by Arend and Goldstein (1987). The difference in appearance between Plates 1 and 2 cannot be accounted for by 1-D approaches, or by theories that explain the COCE by restricting their analysis to the effect of luminance cusps, which are identical in the two displays (see Todorović, 1987). In fact, Growney and Neri (1986) have recently noted, concerning the percept generated by Plate 2 , that alternative models have "difficulty ... in accounting for effects that are not one-dimensional" and that "the appearance of the illusion ... seems also to depend upon more global, two-dimensional characteristics of the stimulus display" (p. 85).

In our model, the cause of the difference in appearance of the two displays is due to the difference in the con- straints that the BC System imposes upon the filling-in process. Figure 18a is a 2-D stimulus representation (Level 1) depicting the standard case of the COCE in Plate 1 . Figure $18 \mathrm{~b}$ describes the activity pattern across the field of circular concentric on-units (Level 2). Figure $18 \mathrm{c}$ describes the activity pattern across the field of boundary contour units (Level 5). The activity pattern at Level 2 generates a filling-in reaction at Level 6 within the boundary compartments at Level 6 that are induced by output signals from Level 5 . Figure $18 \mathrm{~d}$ presents the final filled-in activity pattern across the field of syncytial units at Level 6. The activity is higher in the left rectangle than in the right one, in accordance with the percept. Figures $19 \mathrm{a}-19 \mathrm{~d}$ are the analogous simulations for Plate 2. Figure 19a depicts the stimulus distribution at Level 1, Figure 19b the activity pattern at Level 2, Figure $19 \mathrm{c}$ the activity pattern at Level 5 , and Figure 19d the final filled-in activity pattern at Level 6 . The corresponding activity distributions in Figures 18 and 19 were drawn on the same scale.

The stimulus distribution in the central portion of Figure 19a is identical to the one in Figure 18a. On the other hand, the background activity level of Figure 19a is higher than that of Figure 18a. As a consequence, although a boundary contour forms around the cusp region in Figure 18c, no boundary contour forms around the cusp region in Figure 19c. Thus, filling-in in Figure 18d occurs within a pair of rectangular compartments abutting the cusp, whereas filling-in in Figure 19d can occur around the exterior of the cusp. However, as in the percept, there still remain traces of the luminance cusp along the vertical midline in Figure 19d.

\section{Percept of an Impossible Staircase and Its Contextual} Reduction to the Craik-O'Brien-Cornsweet Effect

Plate 3 presents another visual display involving luminance cusps (from Todorović, 1983); an elaborated octagonal version is displayed and discussed in Todorović (1987). The figure consists of a conjunction of four Lshaped regions on a dark background. The edges between the regions are formed by luminance cusps, and their central portions all have the same luminance level.

The Level 1 representation of this luminance distribution is presented in Figure 20a. The Level 2 activity pattern across the field of on-units is shown in Figure 20b. The Level 5 activity pattern across the field of boundary contour units is illustrated in Figure 20c. This pattern delineates the compartments within which filling-in takes place in Level 6 in response to inputs from Level 2. Figure $20 \mathrm{~d}$ presents the final filled-in activity pattern at Level 6 of the system, which is in accord with the percept.

An interesting property of the display in Plate 3 is revealed when parts of it are occluded. If the upper portion of Plate 3 is screened such that only parts of the bottom two L-shaped figures are visible, the resulting luminance distribution is represented in Figure 21a using a screening performed by an occluder with the luminance of the background. (This is not essential.) Figure 21b displays the filled-in activity profile at Level 6 in response to the 


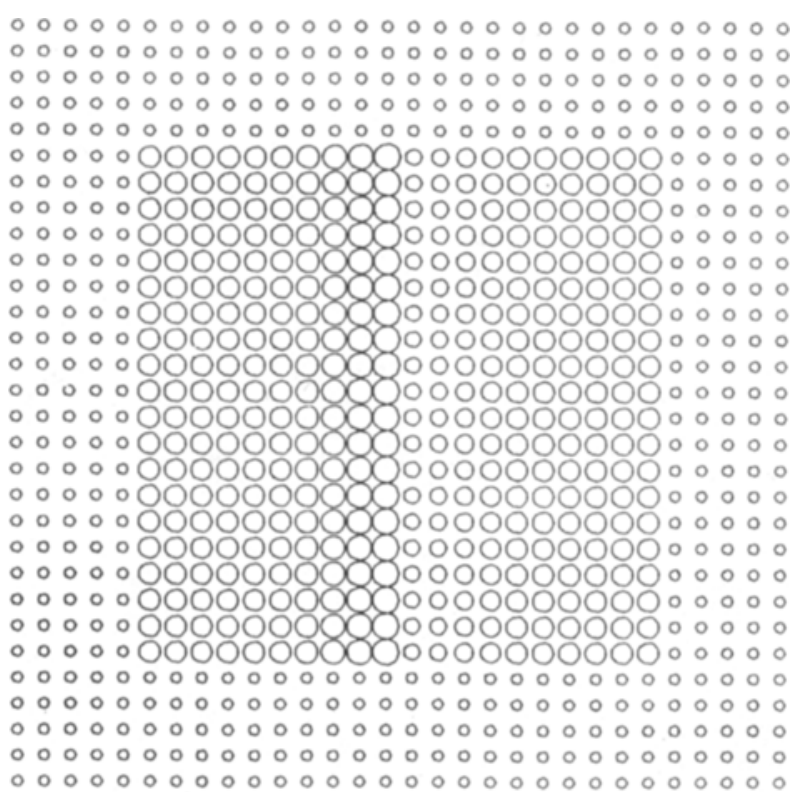

(a)

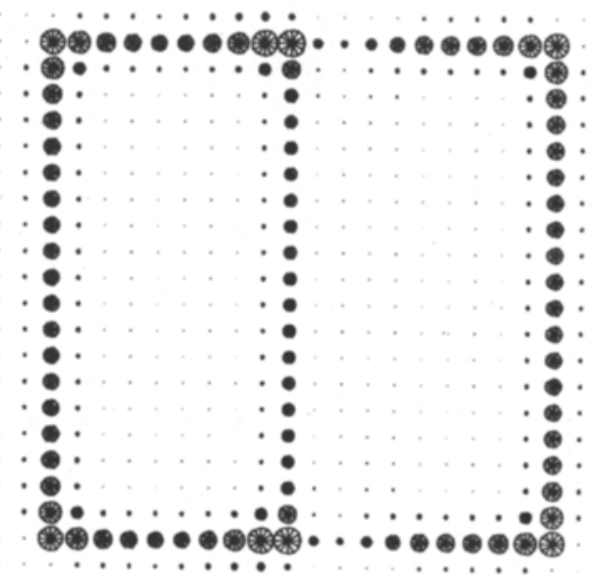

(c)

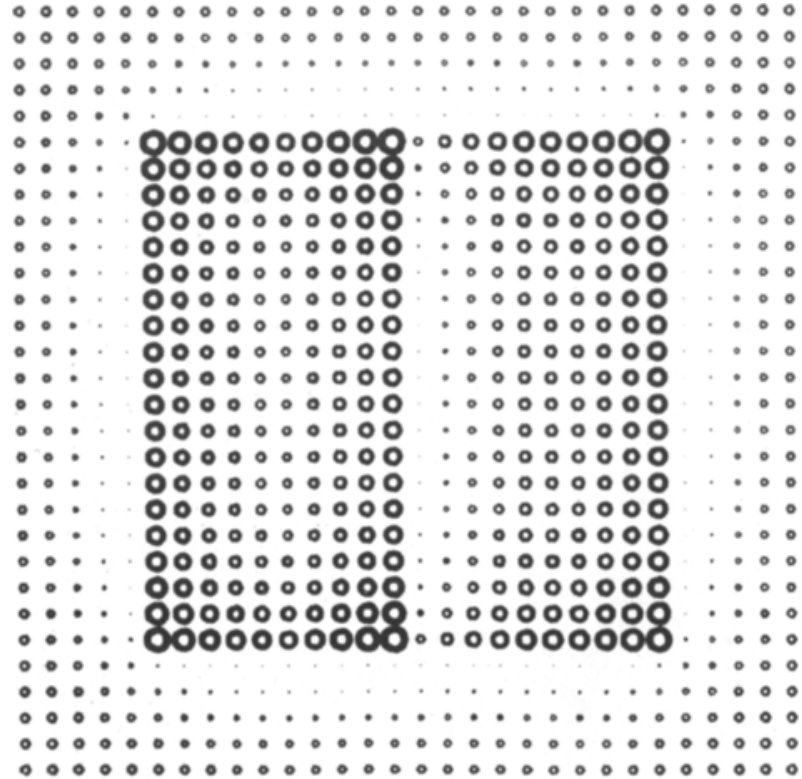

(b)

000000000000000000000000000000 000000000000000000000000000000 000000000000000000000000000000 000000000000000000000000000000 000000000000000000000000000000

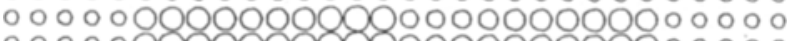

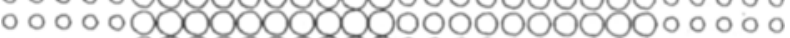
o o o o o 08080898080000000000 o o o o o o o o o 09898989800000000000 o o o o

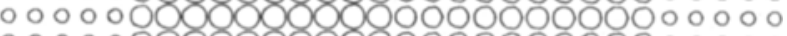
o o o o o 008080800000000000000 o o o o o o o o 08080808000000000000000

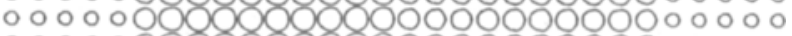

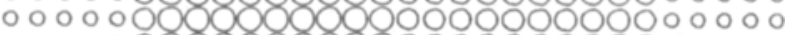
o o o o o 080800808000000000000 o o o

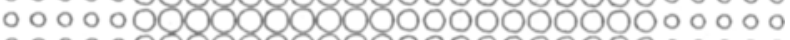

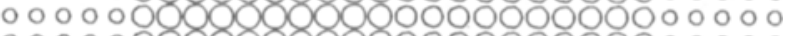
o o o o o 080808080800000000000 o o o o o o o o o 000090000000000000000000

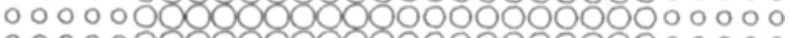

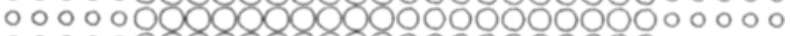
o o o o o 0808090000000000000000

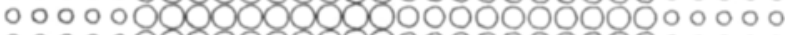

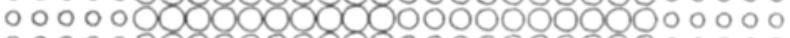
० o o o o 000000000000000000000 o o o 000000000000000000000000000000 000000000000000000000000000000 000000000000000000000000000000 000000000000000000000000000000 000000000000000000000000000000

(d)

Figure 18. The simulation of the 2-D COCE. (a) The stimulus distribution. (b) The on-cell activity profile. (c) The output of the Boundary Contour system. (d) The filled-in syncytium, which predicts the brightness appearance of the stimulus, and should be compared with Plate 1. The parameters for this and all subsequent 2-D simulations are listed in the Appendix.

occluded luminance distribution in Figure 21a. The percept corresponding to Figure $21 \mathrm{~b}$ is a standard case of the COCE wherein the right rectangle looks brighter than the left one. When the occluding procedure is repeated for different parts of Plate 3, the result is a paradoxical set of brightness appearances (Todorović, 1983). If the left portion of Plate 3 is occluded, the remaining top por- tion of the display is brighter than the bottom one. If the bottom is occluded, the remaining left portion is brighter than the right one. Finally, if the right portion is occluded, the bottom portion is brighter than the top one.

This incompatible set of relations is similar to the appearance of the "impossible staircase" by Penrose and Penrose (1958) and Escher (1961). On the other hand, 


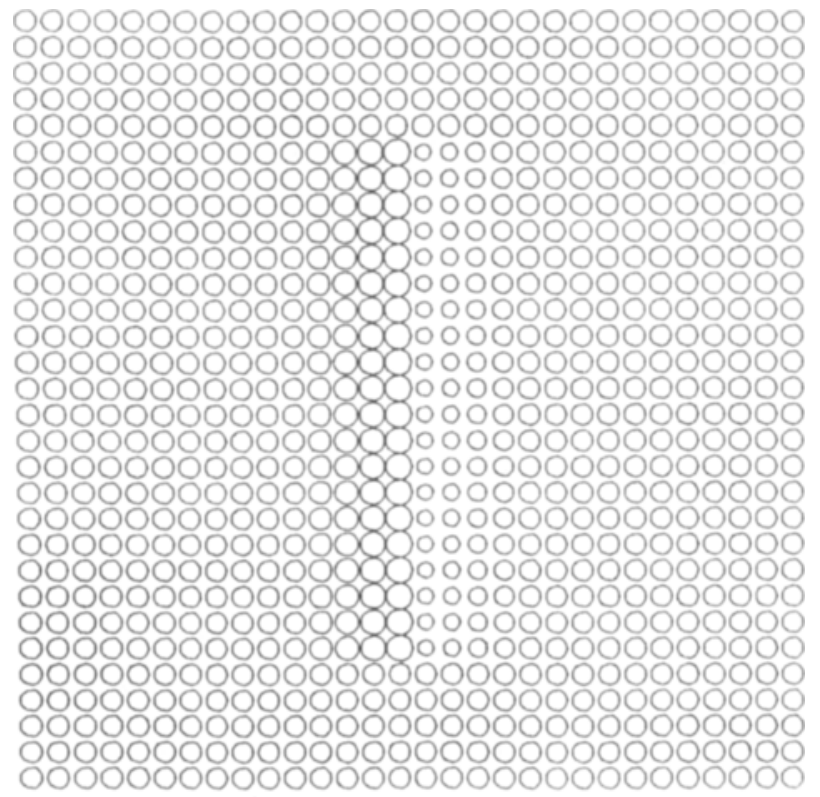

(a)

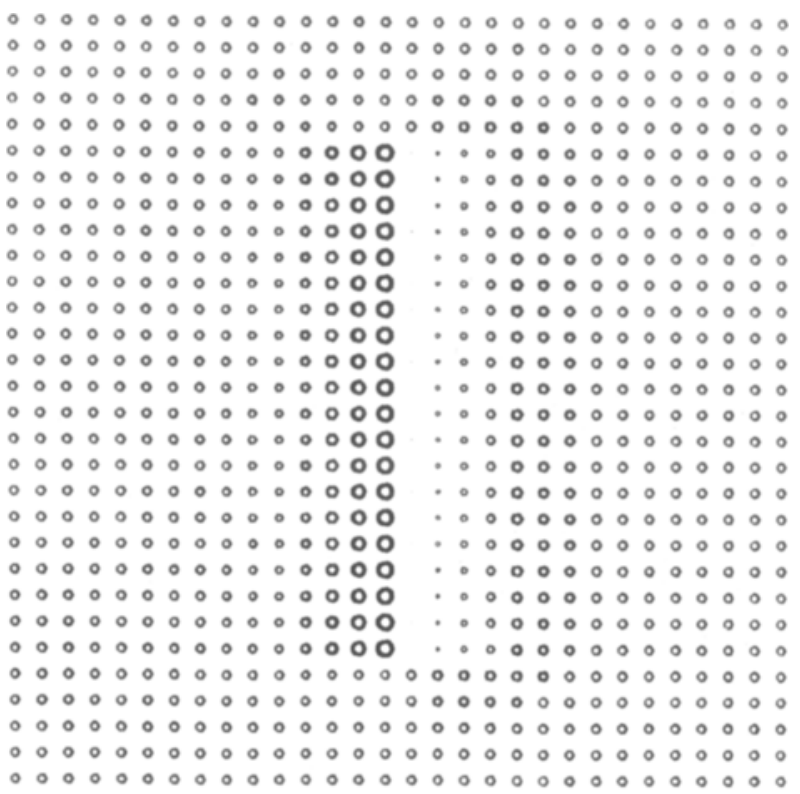

(b)

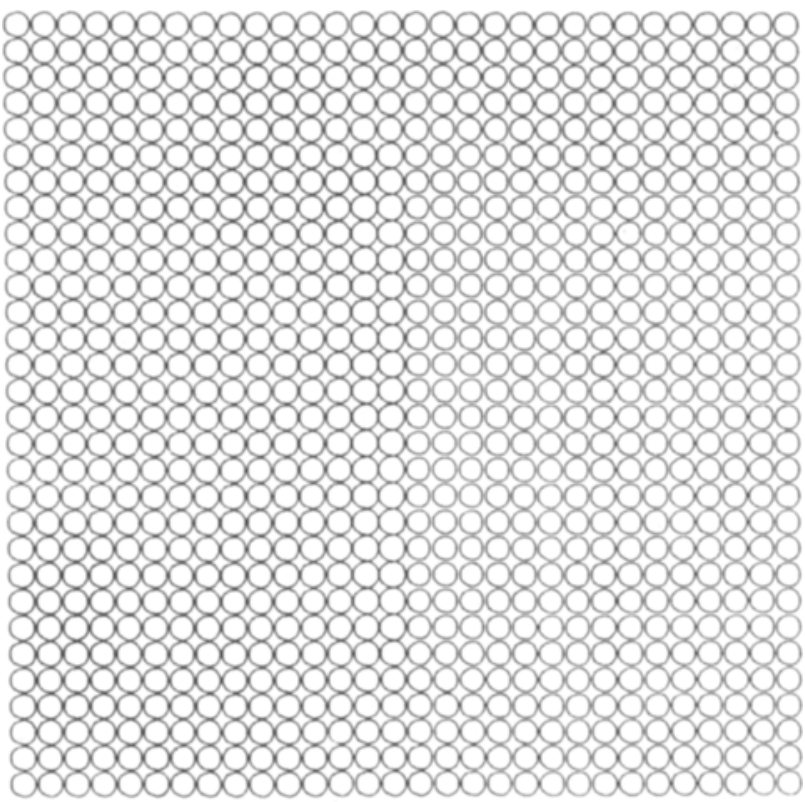

(d)

Figure 19. The simulation of the display in Plate 2. (a) The stimulus disctibution. (b) The on-cell distribution. (c) The output of the Boundary Contour (BC) system. (d) The filled-in syncytium. In contrast to Figure 18, the BC output exhibits no closed compartments. Thus, the filling-process diffuses over the whole extent of the image and no COCE develops. The stimulus vertical cusp survives in the output.

observation of the whole unoccluded display does not induce stable brightness relations; the L-shaped regions partially lose their homogeneous appearance, and the brightness gradients flanking their shared edges become more prominent. These observations have been confirmed experimentally in similar displays by Arend and Goldstein (1987). Our model thus predicts in Figure 20d the incon- clusive appearance of the unoccluded input pattern in Figure 20a, as well as the appearance, in Figure $21 \mathrm{~b}$, of the COCE in response to the occluded input pattern in Figure $21 \mathrm{a}$. In addition, the simultaneous brightness contrast effect perceived when Plate 3 is inspected, which results in reduced brightness of the inner portion of the background, is also obtained in Figure $20 \mathrm{~d}$. 


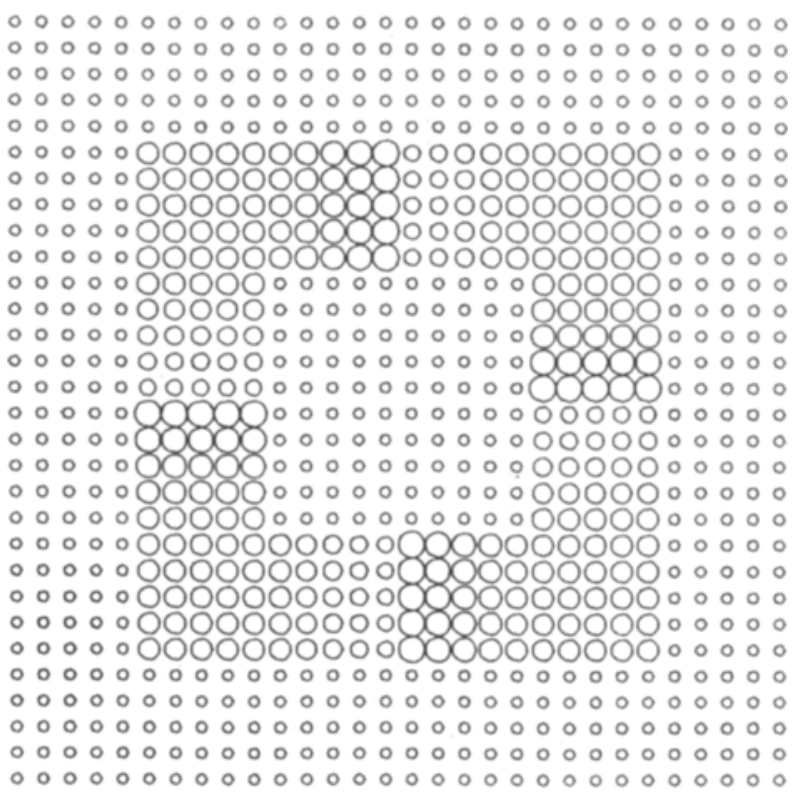

(a)

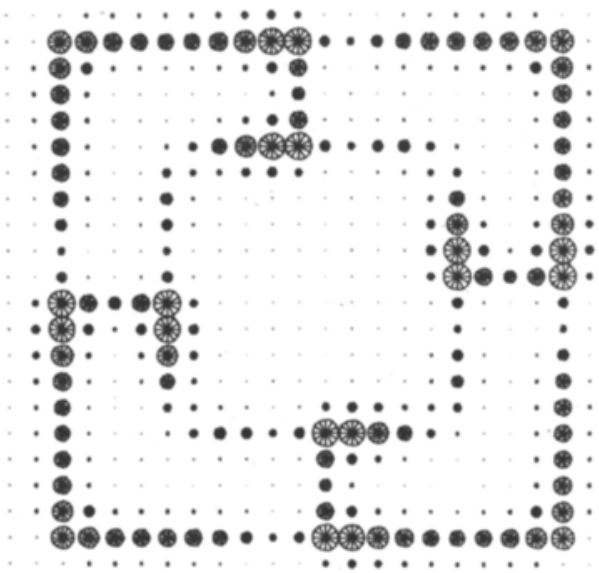

(c)

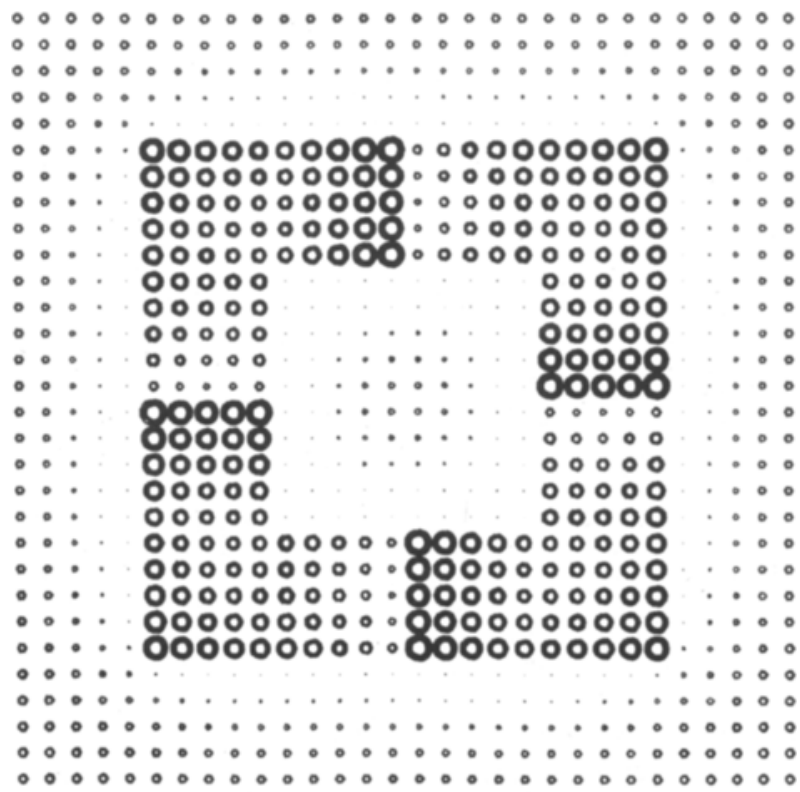

(b)
000000000000000000000000000000 000000000000000000000000000000

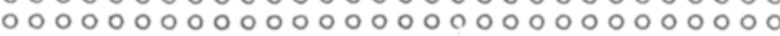
000000000000000000000000000000 000000000000000000000000000000

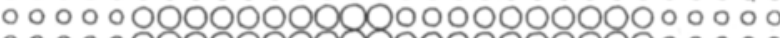

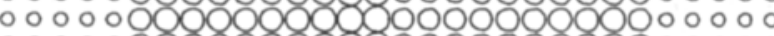
o o o o o o

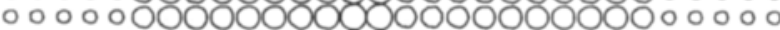

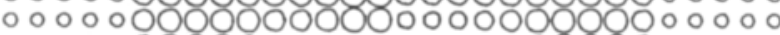

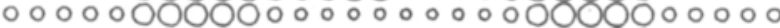

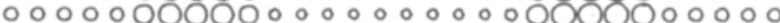

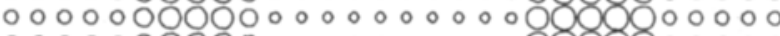

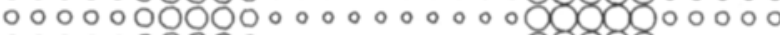
$000000000000 \% 0 \% 00.0808000000$

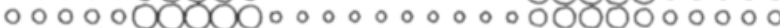

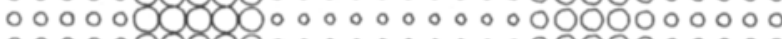

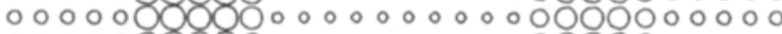
$00000088000 \% 000000000000$

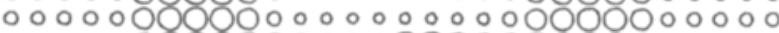

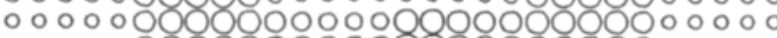
○ o o o o 00000000000000000000 o o o c

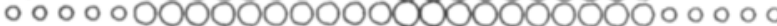

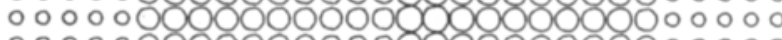
० ० o o o 00000000008000000000 o o o o 000000000000000000000000000000 000000000000000000000000000000 0 0 0 0 0 0 0 0 0 0 0 0 0 0 0 0 00000000000000 000000000000000000000000000000 000000000000000000000000000000

(d)

Figure 20. The simulation of the impossible staircase. (a) The stimulus distribution. (b) The on-cell activity profile. (c) The Boundary Contour output. (d) The filled-in output.

\section{The Koffka-Benussi Ring}

The interaction of $\mathrm{BCs}$ and the filling-in process are well illustrated through simulation of the Koffka-Benussi ring (Koffka, 1935; Berman \& Leibowitz, 1965). The version that we simulate uses a rectangular annulus. The annulus has an intermediate luminance level and is superimposed upon a bipartite background of the same type as in the classical brightness contrast condition, with one half having a high luminance level and the other half a low luminance level (Figure 22a). The percept of such a stimulus is that the annulus is approximately uniform in brightness, although the right and the left halves of the annulus exhibit some brightness contrast. This percept corresponds to the Level 6 activity profile in Figure 22b.

The brightness distribution in the percept can be changed by the introduction of a narrow black line divid- 


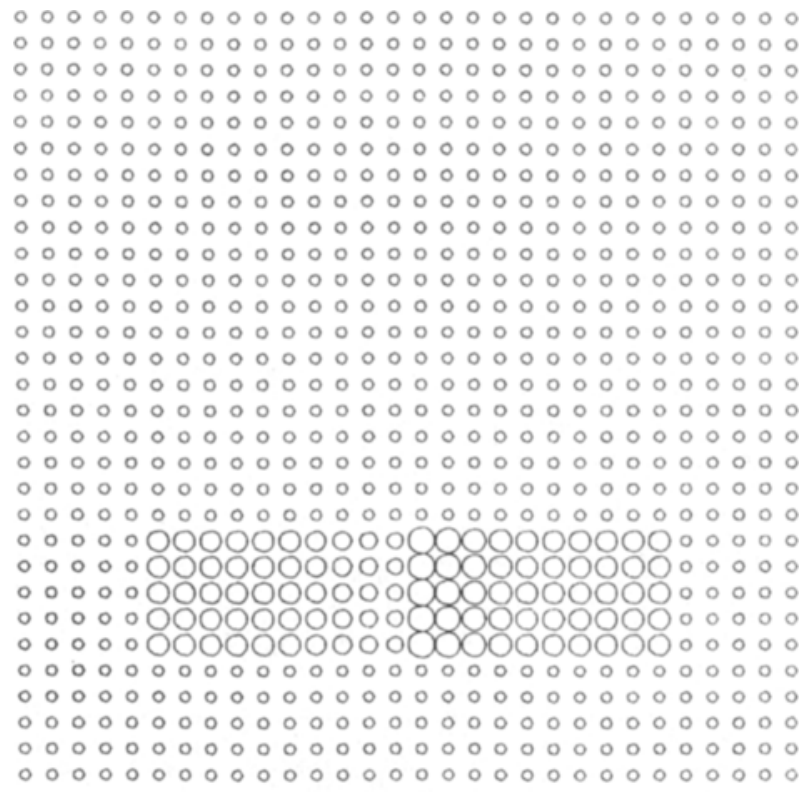

(a)
000000000000000000000000000000 OOOOOOOOOOOOOOOOOOOOOOOOOOOOOO O O O O O O O O O OOOOOOOOOOOOOOOOOOOOO 000000000000000000000000000000 O OOOOOOOOOOOOOOOOOOOOOOOOOOOOO 000000000000000000000000000000

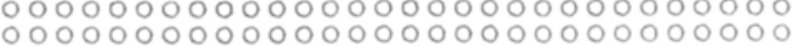

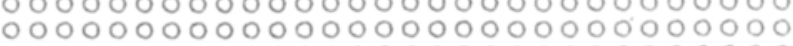
000000000000000000000000000000 000000000000000000000000000000 O OOOOOOOOOOOOOOOOOOOOOOOOOOOOOO 000000000000000000000000000000

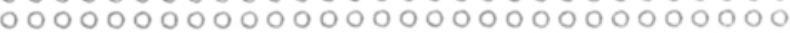
000000000000000000000000000000 O OOOOOOOOOOOOOOOOOOOOOOOOOOOOO 0 O O000000000000000000000000000 000000000000000000000000000000 000000000000000000000000000000

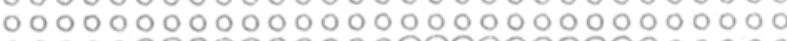

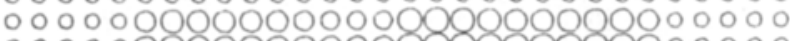

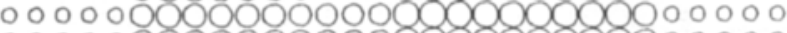

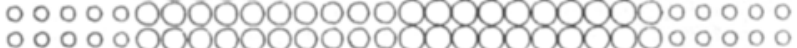
o o o o o obo000000080808008000 o o o 000000000000000000000000000000 000000000000000000000000000000

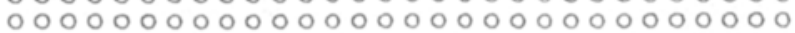
000000000000000000000000000000 000000000000000000000000000000

(b)

Figure 21. The occluded staircase. (a) The stimulus distribution. (b) The filled-in output. The predicted brightness profile is a standard COCE.

ing the stimulus vertically into two halves. Figure $22 \mathrm{c}$ presents this new stimulus distribution. In the percept, as in the Level 6 activity profile (Figure 22d), the annulus is now divided into two regions with homogeneous but different brightnesses that are in accord with brightness contrast.

These effects depend critically upon interactions between contrast, boundaries, and filling-in in the model. In the unoccluded Koffka-Benussi ring, the annular region at Level 6 is a single connected compartment within which diffusion of activity proceeds freely. The opposite contrasts due to the two halves of the background are effectively averaged throughout the annular region, although a residual effect of opposite contrast remains. The introduction of the occluding boundary (Figure 22c) divides the annulus into two smaller compartments (Figure 22d). The different contrasts are now constrained to diffuse within these compartments, generating two homogeneous regions of different brightness.

\section{Anomalous Brightness Differentiation}

Our explanation of the Kanizsa-Minguzzi percept of anomalous brightness differentiation (Figure 1), in which the smaller region looks brighter, is consistent with the explanation of the Koffka-Benussi ring, but also illustrates finer properties of the model. The critical new property, which does not play a significant role in the percept of the Koffka-Benussi ring, is that the spokes in the luminous wheel between the black inner and outer regions induce FC signals as well as BC signals. The FC signals are relatively small relative to those induced by the thick black inner and outer regions. Hence, the perceived brightness difference is small. The $\mathrm{BC}$ signals induced by the spoke divide the wheel-shaped FC System syncytium into two filling-in domains of unequal size. Due to the averaging property of the diffusive filling-in process, the smaller domain possesses a larger average activity than the larger domain. This is because its FC signals due to the spokes are averaged over a smaller region, whereas the FC signals induced by the inner and outer black regions are equally well averaged within each domain. Figure 23 presents the simulation of a rectangular version of the Kanizsa-Minguzzi stimulus distribution (Figure 23a) and the filled-in Level 6 activity profile (Figure 23b).

It is instructive to contrast the model properties that give rise to the Koffka-Benussi percepts and the KanizsaMinguzzi percept. In the Koffka-Benussi percepts, the contrasts induced by the background (Figures 22a and 22c) are asymmetric with respect to the figure and massive due to their extent along the figure perimeter. The main effect of the thin vertical line (Figure 22c) is to induce a new BC which divides the figure into two filling-in domains of equal size. The FCs caused by this vertical line are swamped by the filled-in contrasts induced by the background within these equally large regions. In the Kanizsa-Minguzzi demonstration (Figure 23), the contrasts induced by the background are symmetric with respect to the figure. On the other hand, the additional lines divide the figure into unequal filling-in domains. Hence the influence of these lines as inducers of FC signals can cause a perceived, albeit small, brightness difference. 


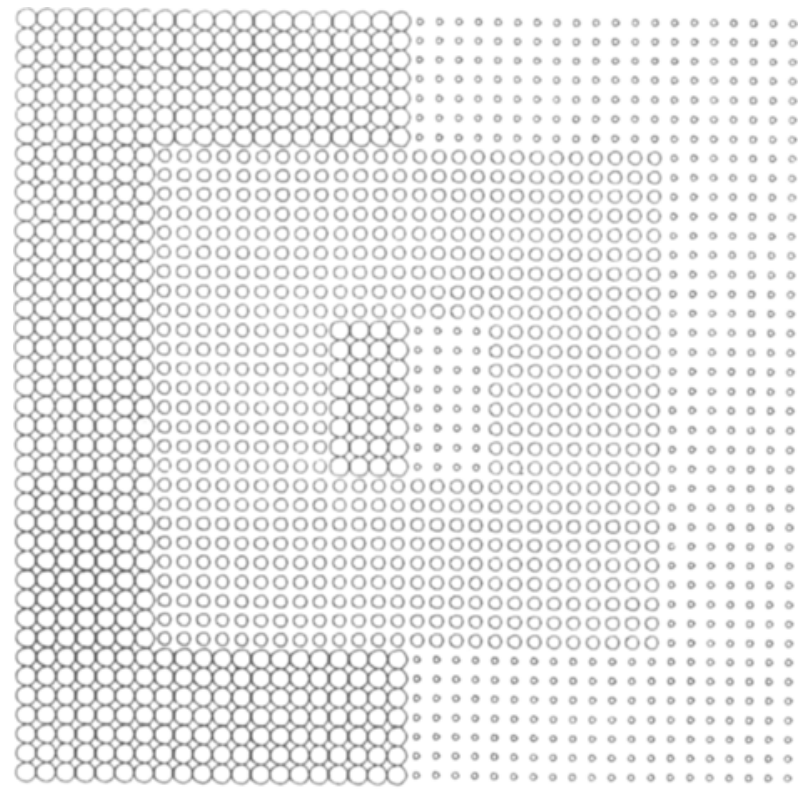

(a)

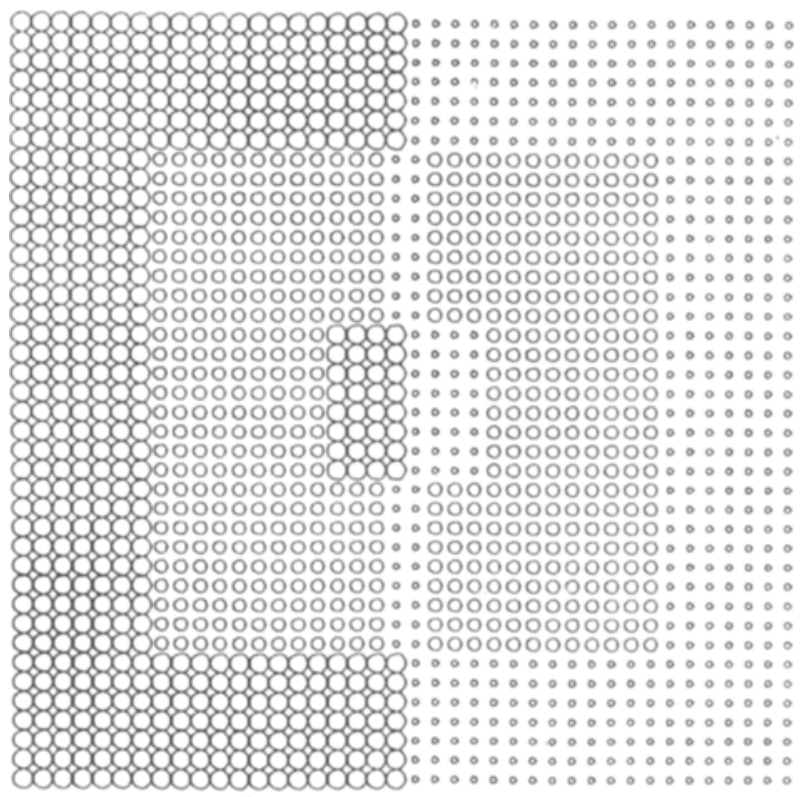

(c)

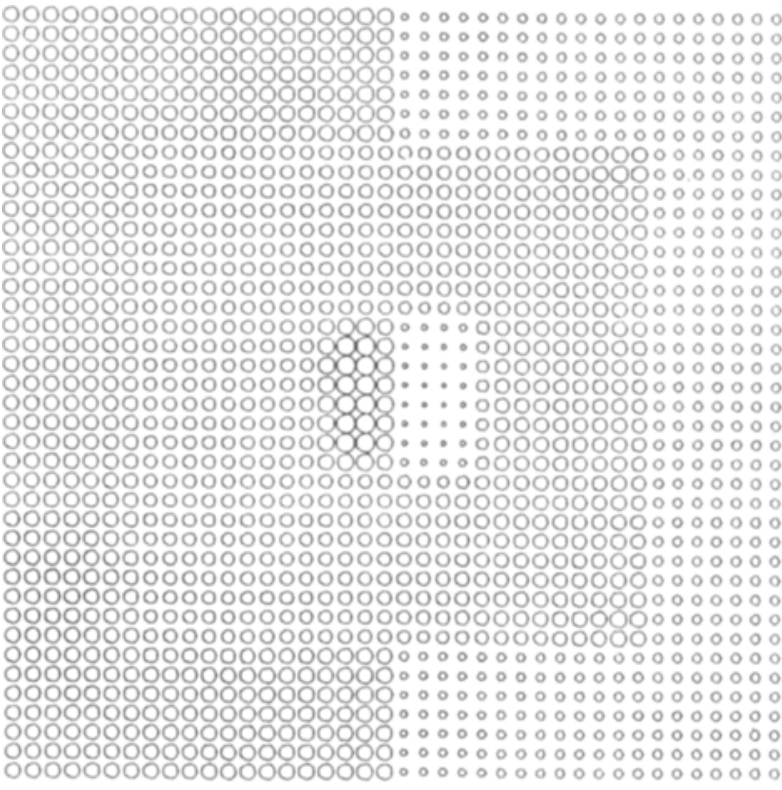

(b)

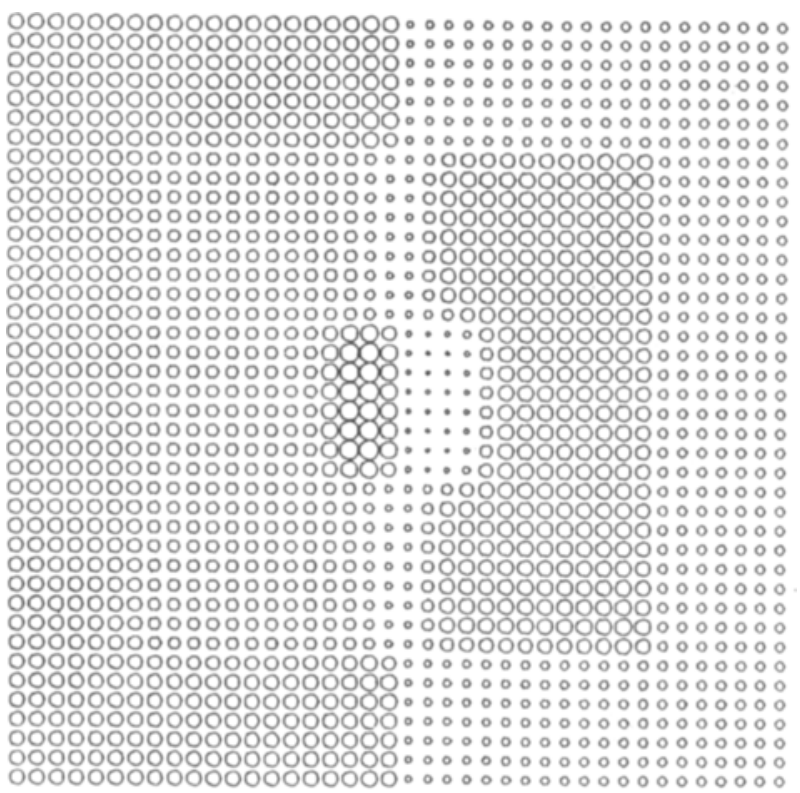

(d)

Figure 22. The Koffka-Benussi ring. (a) The stimulus distribution corresponding to the homogeneous undivided square annulus of medium luminance on a bipartite background. (b) The filled-in output corresponding to the stimulus in (a). (c) The same stimulus distribution as in (a), except that the annulus is here divided by vertial short dark lines into two equiluminant halves. (d) The filled-in output corresponding to the stimulus in (b). The two halves of the annulus are homogeneous and have different brightness levels.

\section{Mondrian Percepts under Constant and Variable Illumination}

Shapley (1986) presented an achromatic Mondrian display which the Land $(1977,1986)$ retinex theory cannot adequately explain. Since the retinex theory was devised with Mondrian displays as a primary explanatory target, the Shapley (1986) demonstration represents a serious challenge to the retinex theory of brightness perception.

A stimulus of this type is represented in Figure 24a. Consider the two squares in Figure 24a, the first near the top left corner and the second near the bottom right corner, which have the same size and luminance. Despite these 


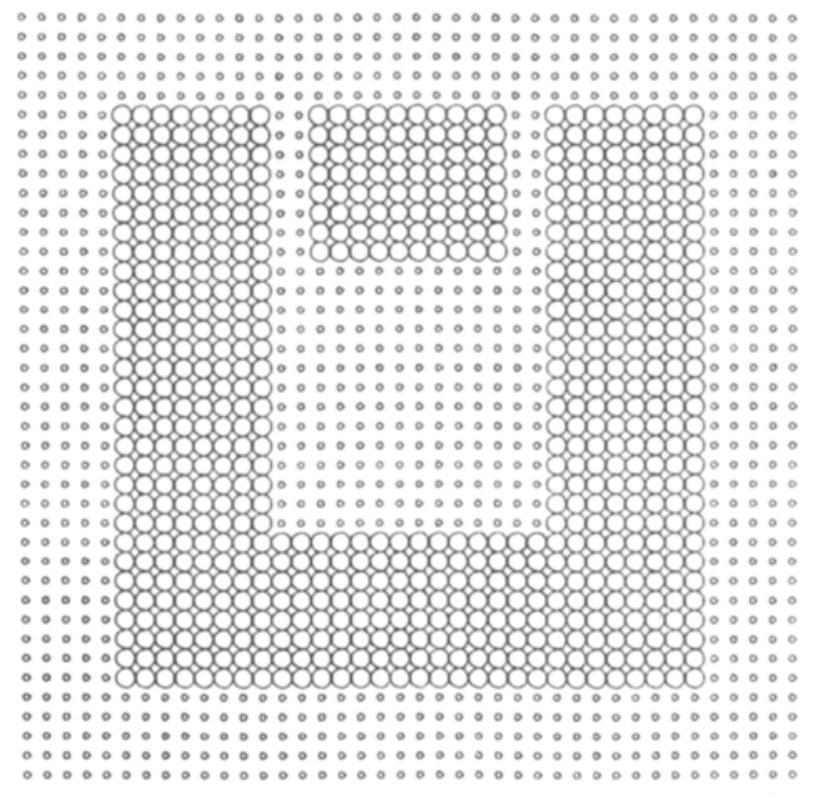

(a)

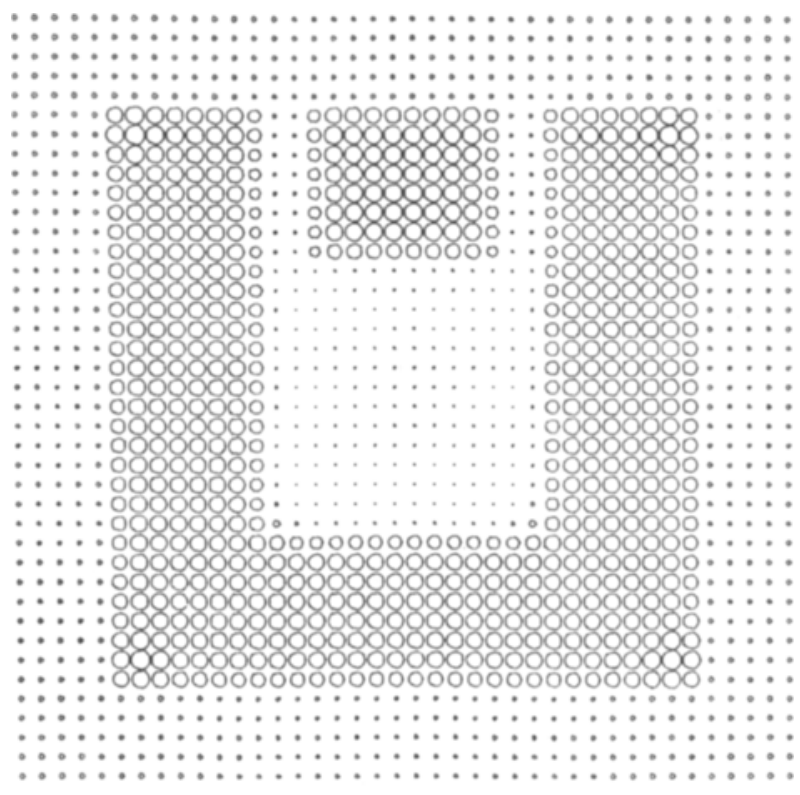

(b)

Figure 23. The simulation of the Kanizsa-Minguzzi anomalous brightness differentiation. (a) The stimulus distribution of a rectangular version of the display. (b) The filled-in output. The small segment is correctly predicted to appear brighter than the large segment.

equalities, the filled-in activity profile of the upper square is more intense than that of the lower square, corresponding to the percept that the upper square is brighter.

This brightness difference is due to the following combination of factors in our model. The luminances of the regions surrounding the two squares were chosen such that, on the average, the upper square is more luminant than its surround and the lower square is less luminant than its surround. In consequence, as can be seen in Figure $24 \mathrm{~b}$, more Level 2 on-unit activity is present within the region corresponding to the upper square. The on-unit activity diffuses within the compartments delineated by the BCs (Figure 24c). Thus, in the filledin upper square of Figure 24d, a larger amount of activity is spread across the same area as in the lower square, thereby explaining the final brightness difference.

Figure 24 thus illustrates how the present model can explain a Mondrian percept that falls outside the explanatory range of retinex theory. However, one of the important accomplishments of retinex theory was to discount the illuminant in response to an unevenly illuminated Mondrian. The image represented by Figure 24a was evenly illuminated. A simple 1-D example of how our model discounts the illuminant was shown in Figure 10b. We now show that the model can discount the illuminant in response to unevenly illuminated Mondrians, indeed in response to Mondrians whose brightness profile in even illumination is not explicable by retinex theory.

Imagine that the Mondrian in Figure 24 is illuminated by a gradient of light that decreases linearly across space from the lower right corner of the figure. The resulting luminance distribution is depicted in Figure 25a. The up- per square now receives, on the average, less luminance than the lower square. Despite this fact, the filled-in activity profile of the upper square at Level 6 is more intense than that of the lower square (Figure 25d). Figures $24 \mathrm{~b}$ and $25 \mathrm{~b}, 24 \mathrm{c}$ and $25 \mathrm{c}$, and $24 \mathrm{~d}$ and $25 \mathrm{~d}$ are, in fact, virtually indistinguishable, thereby illustrating effective discounting of the illuminant in this particular situation. This successful result does not, however, imply that complete discounting will occur in response to all combinations of achromatic and chromatic images, illuminants, and bounding regions (Arend \& Reeves, 1986). The systematic analysis of all these factors is a topic for future research.

\section{The Hermann Grid: A Transitional Example}

The appearance of darker spots at the intersections of bright streets in the Hermann grid is a perceptual phenomenon with a generally accepted physiological foundation related to the activity of cells with concentric antagonistic receptive fields (Baumgartner, 1960). Within our model, some additional issues surrounding this phenomenon are indicated. Figure 26a is the luminance distribution of a small portion of the grid containing four streets and four intersections. Figure 26b depicts the level of activity of concentric on-units. Consistent with the standard explanation, there is a reduced level of activity associated with street intersections, compared with units located within the streets, because units in a street intersection are more inhibited than units within streets. However, in our model it is not Level 2 but Level 6 at which brightness is determined. Figure $26 \mathrm{~d}$ presents the Level 6 distribution, showing that the difference of ac- 


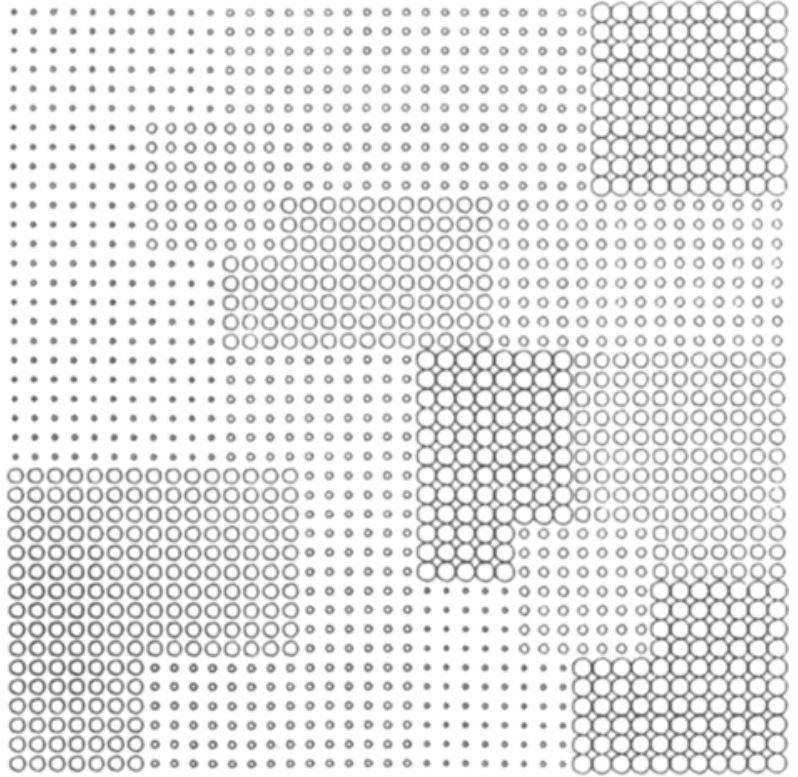

(a)

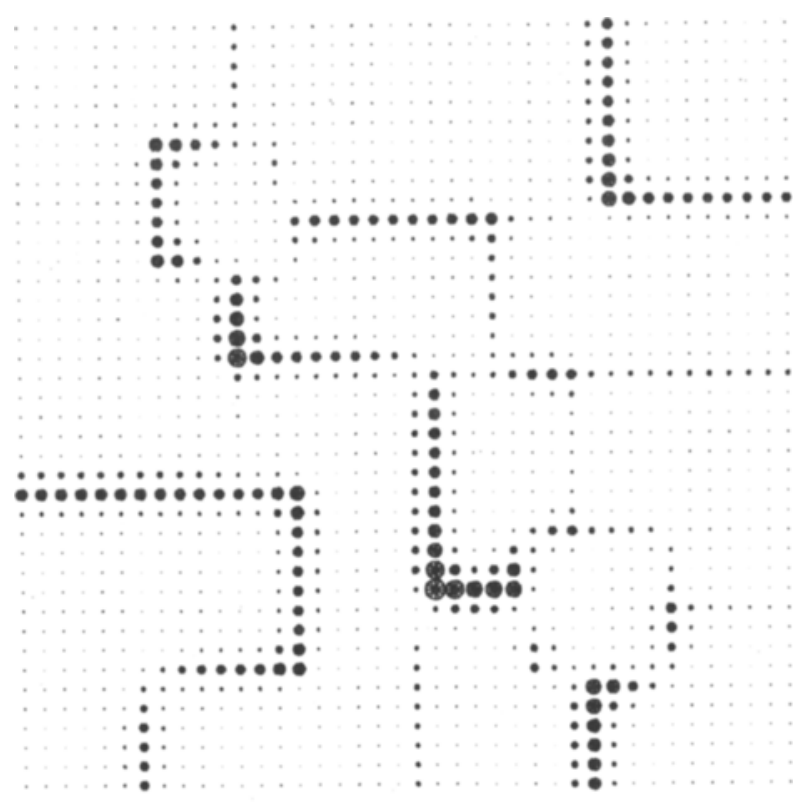

(c)

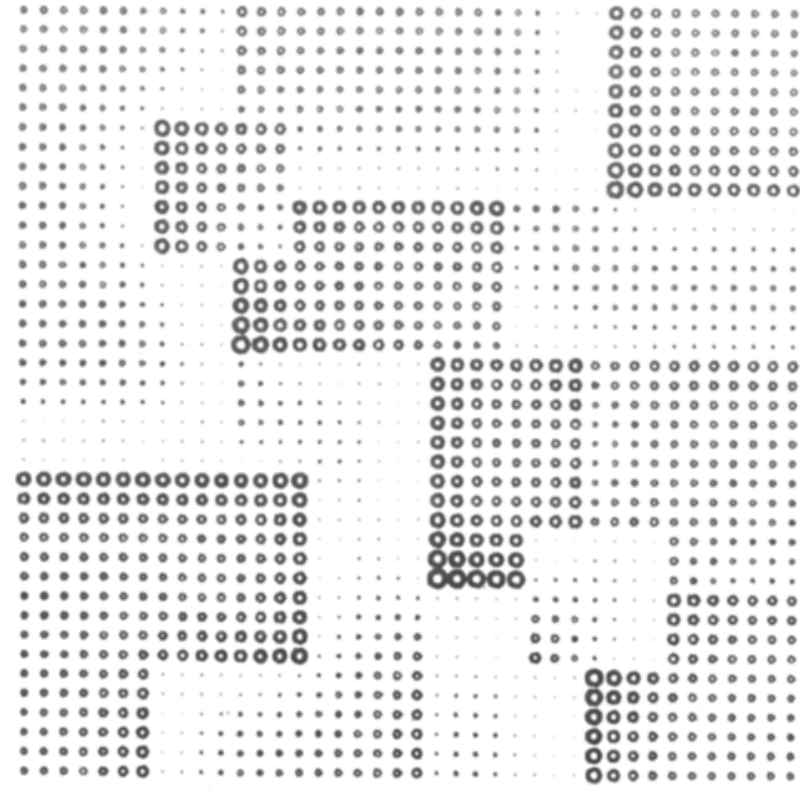

(b)

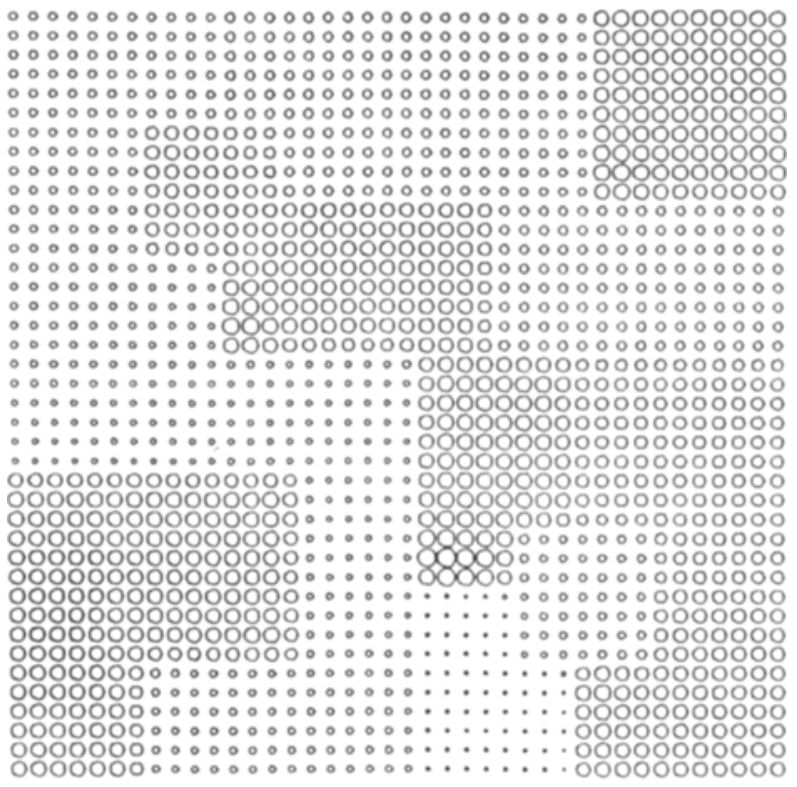

(d)

Figure 24. The evenly illuminated Mondrian. (a) The stimulus distribution consists of 13 homogeneous polygons with 4 luminance levels. Note that the square in the upper left portion of the stimulus has the same luminance as the square in the lower right portion. However, the average luminance of the regions surrounding the lower square is higher than the corresponding average luminance for the upper square. (b) The on-cell distribution. The amount of on-cell activity within the upper square is higher than within the lower square. (c) The Boundary Contour output. (d) The filled-in syncytium. The upper square is correctly predicted to look brighter than the lower square.

tivities between streets and street intersections is preserved at this level.

Inspection of Figure 26c, the BC output, reveals one reason for this effect. Since the streets are very narrow, the BCs from both sides extend into the middle of the streets, throughout their width and length, except at the intersection regions. Therefore activity at the intersections is blocked from diffusing through the streets and vice versa, and both streets and intersections retain the relative activity levels inherited from the on-unit level.

The boundaries are computed in Figure 26c using the same parameters as in all the 2-D simulations in this sec- 


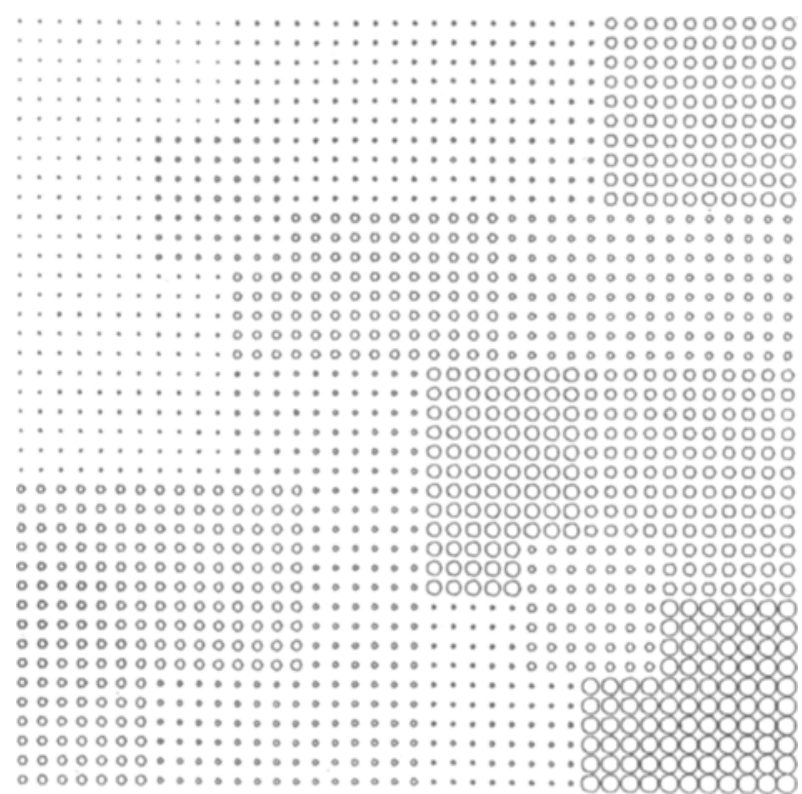

(a)

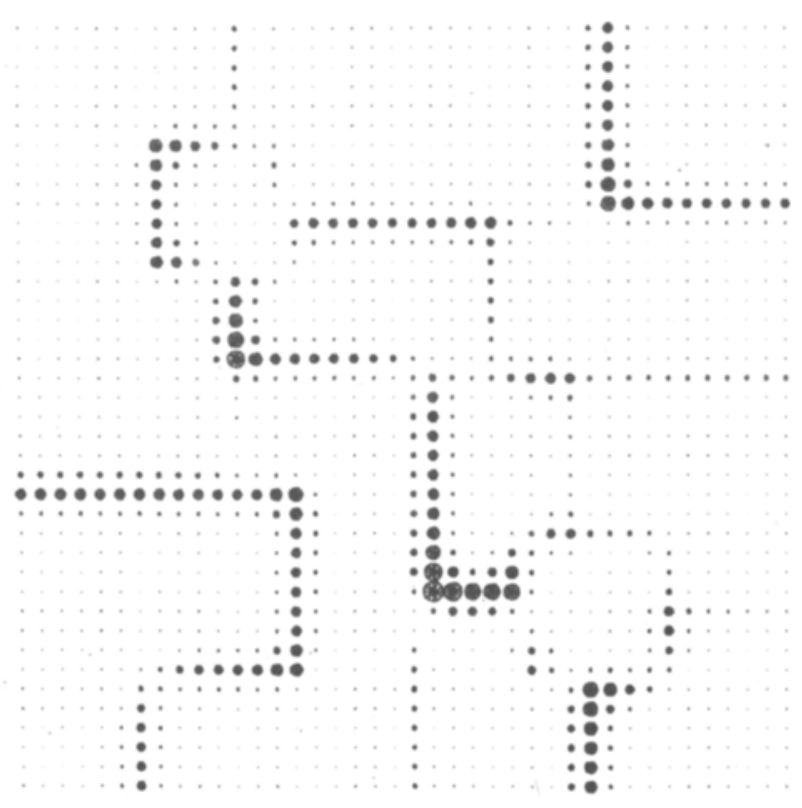

(c)

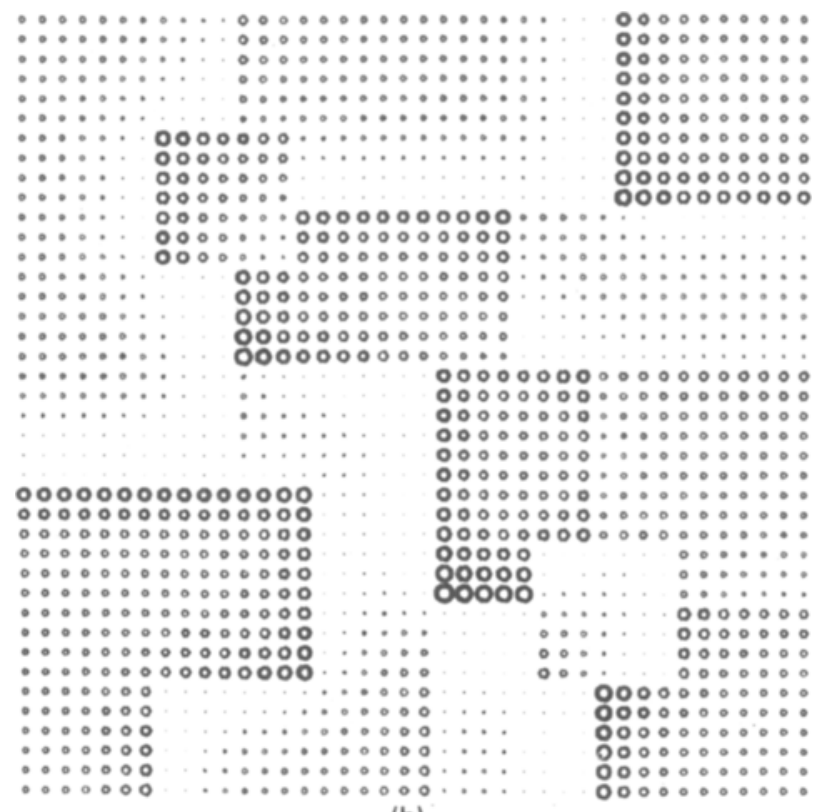

(b)

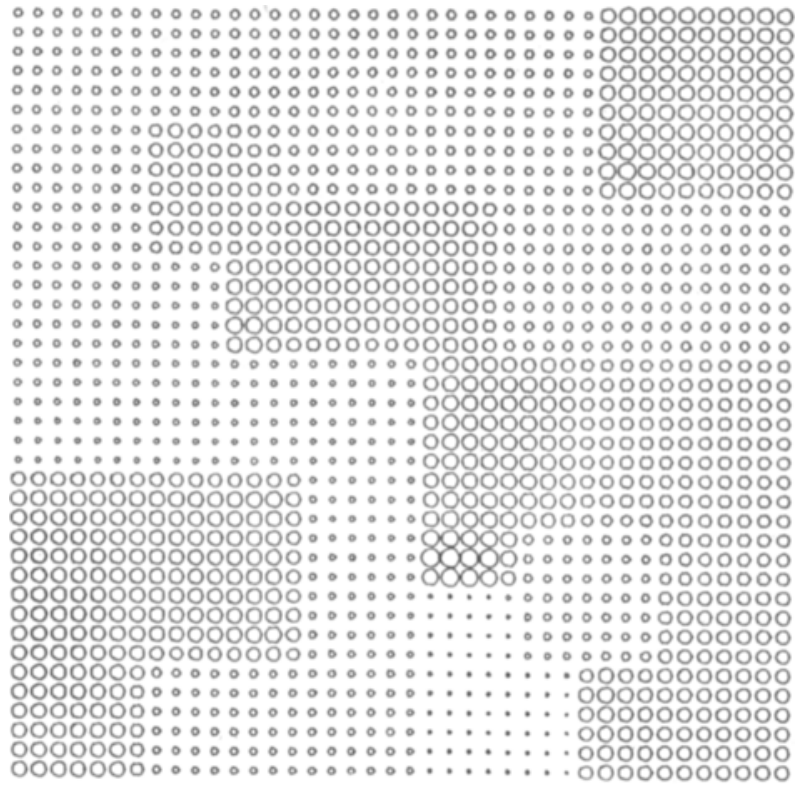

(d)

Figure 25. The unevenly illuminated Mondrian. (a) The stimulus distribution simulates the transformation of Figure 24a caused by the presence of a light source whose intensity decreases linearly from the lower right corner toward the upper left corner of the stimulus. The lower square is now more luminant than the upper square. (b) The on-cell distribution. (c) The Boundary Contour output. (d) The filled-in syncytium. Figures $25 \mathrm{~b}, \mathbf{2 5 c}$, and 25d are very similar to the corresponding figures for the evenly illuminated Mondrian (Figure 24). This illustrates the model's discounting of the illuminant. In addition, the upper square is still predicted to appear brighter than the lower square, which is another instance of contrast constancy (see Figure 12d).

tion (see the Appendix). Figure 26c thus illustrates that BCs may generate a plexus, or boundary web, of small compartments that prevent filling-in from spreading substantially beyond the locations of their on-cell inputs. Such boundary webs are also important, for example, in explaining aspects of 3-D surface perception (Grossberg \&
Mingolla, 1987). In Figure 26c, the total thickness of each street influences whether filling-in will be trapped within that street.

The above discussion illustrates that BCs may exist at locations that do not correspond exactly to image contrasts. In order to obtain a more complete explanation of 


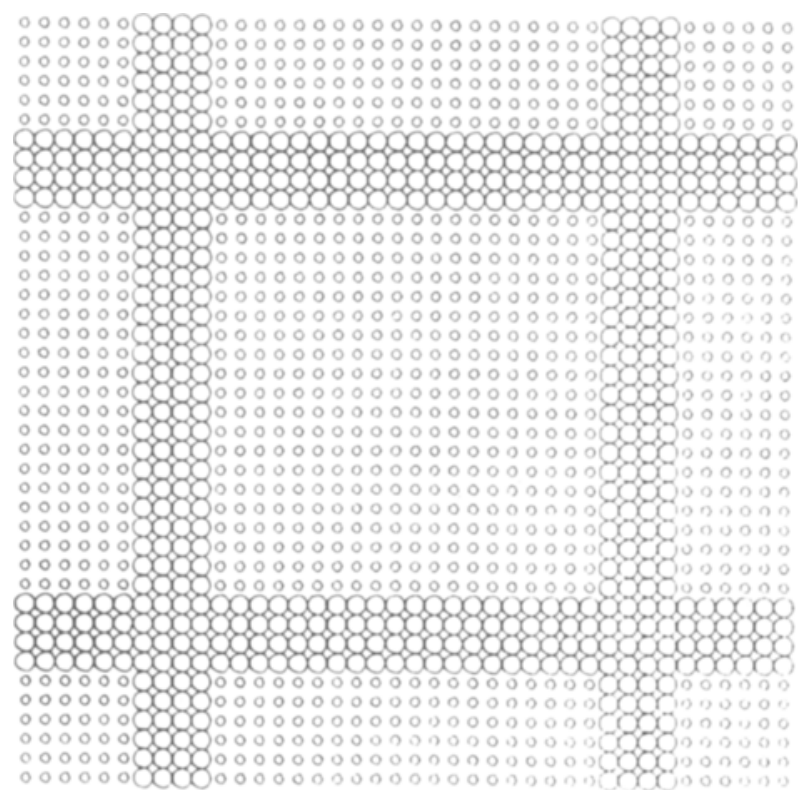

(a)

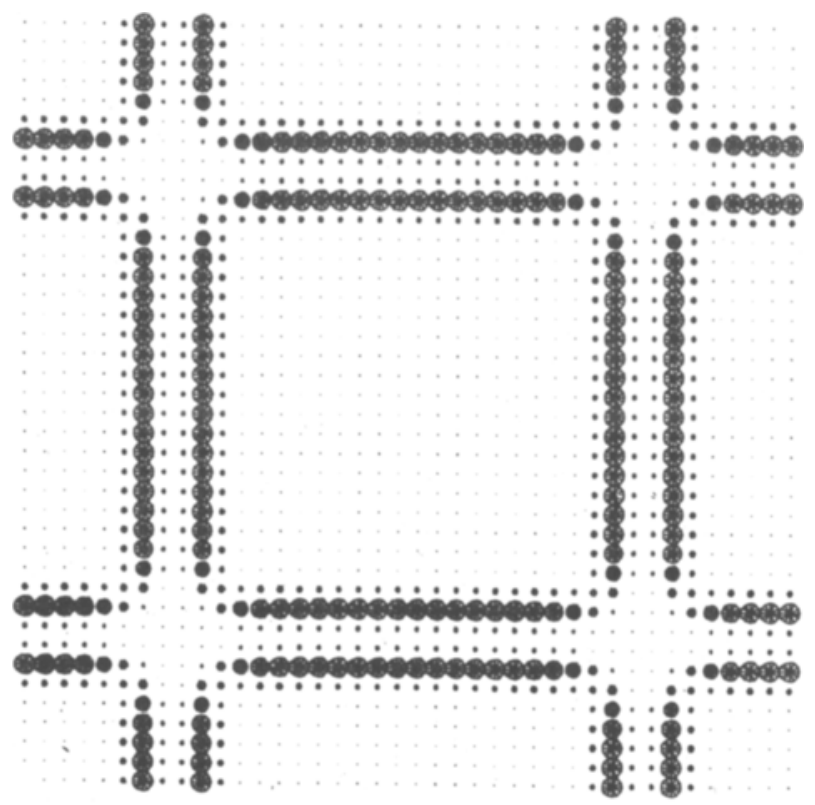

(c)

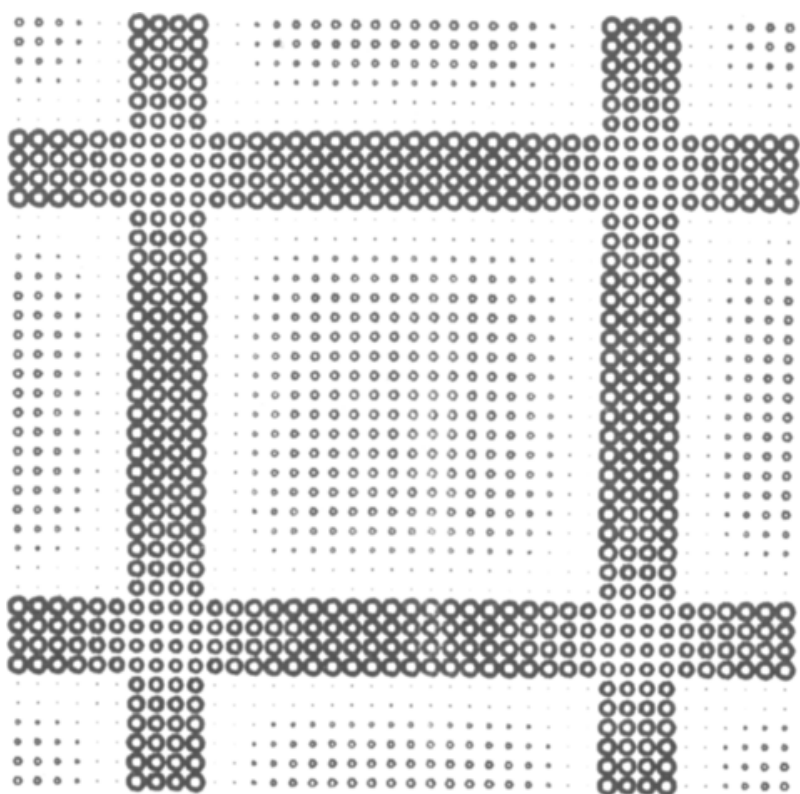

(b)

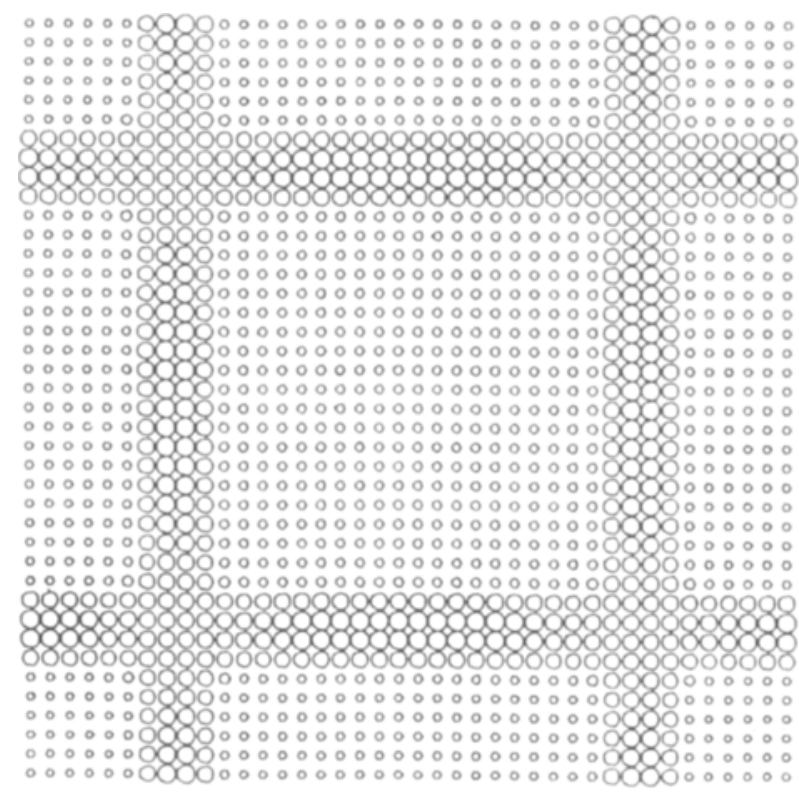

(d)

Figure 26. The Hermann grid. (a) The stimulus distribution depicts a portion of the Hermann grid containing four bright lines (streets) on a dark surround. (b) The on-cell distribution. The activity of units at street intersections is lower than within streets, as predicted by the standard theory of this phenomenon (Baumgartner, 1960). (c) The Boundary Contour output. Note that boundary contour activity is present along the streets but not at intersections. (d) The filled-in syncytium. The intersections are predicted to look darker than the streets. See text for details.

the Hermann grid, this possibility needs to include a process of emergent boundary segmentation. As noted in Part 1, the implementation of such a segmentation process lies outside the scope of this article. With emergent boundary-segmentation mechanisms appended, one would also need to analyze how and when BCs colinearly cooperate from street to street across their intersections, and compete at each location across orientations, to create emergent boundary configurations in which brightness or color can flow. Grossberg and Mingolla (1985a, 1985b) 
have analyzed a number of achromatic and chromatic percepts that are strongly influenced by such emergent boundary segmentations.

\section{PART 6 DISCUSSION}

The computer implementation of the model described in this paper has a limited domain of application since it deals only with monocular achromatic brightness effects. Extensions into the chromatic and binocular domains have been described in Grossberg (1987a, 1987b). Brightness can also be influenced by emergent segmentations that are not directly induced by image contrasts, as in Kanizsa's illusory triangle, the Ehrenstein illusion, and neon color spreading effects. These and related grouping and segmentation effects have been discussed by Grossberg and Mingolla (1985a, 1985b). Their implementation includes a version of the BC System in which emergent segmentations can be generated through lateral interactions between oriented channels. Such interactions may play a role in the orientation-sensitive brightness effects reported by McCourt (1982), Sagi and Hochstein (1985), and White (1979). The implementation in this paper also omits possible effects of multiple-scale processing, as the receptive fields of all units within a network were assumed to have a single receptive field size. Units of multiple sizes may be involved in the explanation of classical brightness assimilation (Helson, 1963). Grossberg and Mingolla (1987) have studied the role of multiple scales in the perception of 3-D smoothly curved and shaded objects. A number of depth-related effects, such as in phenomena of transparency (Metelli, 1974) and proximity-luminance covariance (Dosher, Sperling, \& Wurst, 1986) have been discussed by Grossberg (1987a, 1987b). The model in its current form also does not treat the temporal variations of brightness due to image motion (Cavanagh \& Anstis, 1986; Todorović, 1983, 1987) or stabilization (Krauskopf, 1963; Pritchard, 1961; Yarbus, 1967). Finally, the application of the model to natural noisy images has yet to be accomplished.

In sum, the system described in this paper does not attempt to explain the complete gamut of brightness phenomena. These limitations are not, however, insurmountable obstacles; rather, they point to natural extensions of the model, many of which have been discussed and implemented in related work. However, even the processing of brightness in monocular, achromatic, static, noise-free images is full of surprising complexities. Only a model capable of handling these basic phenomena can be a foundation upon which still more complex effects can be explained.

Surprisingly little computationally oriented work has been devoted to these fundamental aspects of visual perception. Several contemporary algorithms were influenced by Land's seminal work (Blake, 1985; Frisby, 1979; Horn, 1974). Other computational models have provided alternative approaches to the analysis of filling-in (Arend
\& Goldstein, 1987; Hamada, 1984). Our model has been used to simulate a much larger set of brightness data, and includes mechanistic explanations of classical longstanding phenomena described in every review of brightness processing, recently discovered but unexplained data, and predictions of yet untested phenomena, including predictions of testable patterns of physiological activation.

The direct measurement of spatial distributions of activity in real neural networks is still hampered by considerable technical difficulties. Recently, a number of procedures have been developed to enable the visualization of the spatial patterning of activation across large numbers of neurons to a given stimulus (Blasdel \& Salama, 1986; Fox et al., 1986; Grinvald, Leike, Frostig, Gilbert, \& Wiesel, 1986; Nothdurft \& Lee, 1982). These new techniques offer exciting possibilities for the study of integrating brain functions. Our computer simulations illustrate that these spatial patterns can be qualitatively different in response to the same stimulus at different levels of functional organization, and predict the types of activation patterns that should be elicited by images that are easily acquired and used in the laboratory. Of particular importance to brightness theory will be the discovery and mechanistic characterization of the cortical fillingin domains whose properties have been so essential to the success of our computer simulations of brightness percepts.

\section{REFERENCES}

AREND, L. E. (1973). Spatial differential and integral operations in human vision: Implication of stabilized retinal image fading. Psychological Review, 80, 374-395.

Arend, L. E., Buehler, J. N., \& Lockhead, G. R. (1971). Difference information in brightness perception. Perception \& Psychophysics, 9, 367-370.

AREND, L. E., \& GolDsteIN, R. E. (1987). Lightness models, gradient illusions, and curl. Perception \& Psychophysics, 42, 65-80.

AREND, L. E., \& REEves, A. (1986). Simultaneous color constancy. Journal of the Optical Society of America, A3, 1743-1751.

Baumgartner, G. (1960). Indirekte Grössenbestimmung der receptiven Felder der Retina beim Menschen mittels der Hermannschen Gittertäuschung (Abstract). Pflügers Archi für die Gesamte Physiologie, 272, 21-22.

BECK, J. (1966a). Effect of orientation and of shape similarity on perceptual grouping. Perception \& Psychophysics, 1, 300-302.

BECK, J. (1966b). Perceptual grouping produced by changes in orientation and shape. Science, 154, 528-540.

Berman, P. W., \& Leibowitz, H. W. (1965). Some effects of contour on simultaneous brightness contrast. Journal of Experimental Psychology, 69, 251-256.

BLAKE, A. (1985). Boundary conditions for lightness computation in Mondrian world. Computer Vision, Graphics, \& Image Processing, 14, 314-327.

Blasdel, G. G., \& Salama, G. (1986). Voltage-sensitive dyes reveal a modular organization in monkey striate cortex. Nature, 321, 579-585.

Braitenberg, V., \& BraitenberG, C. (1979). Geometry of orientation columns in the visual cortex. Biological Cybernetics, 33, 179-186.

BridgemAN, B. (1983). Isomorphism is where you find it. Behavioral \& Brain Sciences, 6, 658-659.

Cavanagh, P., \& Anstis, S. M. (1986). Brightness shift in drifting ramp gratings isolates a transient mechanism. Vision Research, 26, 899-908.

Cohen, M. A., \& Grossberg, S. (1984). Neural dynamics of bright- 
ness perception: Features, boundaries, diffusion, and resonance. Perception \& Psychophysics, 36, 428-456.

CORNSWEet, T. N. (1970). Visual perception. New York: Academic Press.

Davidson, M., \& Whiteside, J. A. (1971). Human brightness perception near sharp contours. Journal of the Optical Society of America, 61, 530-536.

DeValois, R. L., Albrecht, D. G., \& Thorell, L. G. (1982). Spatial frequency selectivity of cells in macaque visual cortex. Vision Research, 22, 545-559.

Dosher, B. A., Sperung, G., \& Wurst, S. (1986). Tradeoffs between stereopsis and proximity luminance covariance as determinants of perceived 3-D structure. Vision Research, 26, 973-979.

Enroth-Cugell, C., \& Robson, J. G. (1966). The contrast sensitivity of retinal ganglion cells of the cat. Journal of Physiology, 187, 517-552.

ENRoth-Cugell, C., \& RoBson, J. G. (1984). Functional characteristics and diversity of cat retinal ganglion cells. Investigative Ophthalmology \& Visual Science, 25, 250-267.

Escher, M. C. (1961). The graphic work of M. C. Escher. London: Oldburne.

Fechner, G. T. (1889). Elemente der Psychophysik (2nd ed.). Leipzig: Breitkopf \& Härtel.

Foster, D. H. (1983). Experimental test of a network theory of vision. Behavioral \& Brain Sciences, 6, 664-665.

Fox, P. T., Mintun, M. A., Raichle, M. E., Miezin, F. M., Allman, J. M., \& VAN Essen, D. C. (1986). Mapping human visual cortex with positron emission tomography. Nature, 323, 806-809.

FrisBy, J. P. (1979). Seeing: Illlusion, brain, and mind. Oxford: Oxford University Press.

FrY, G. A. (1948). Mechanisms subserving simultaneous brightness contrast. American Journal of Optometry and Archives of the American Academy of Optometry, 25, 162-178.

Gerrits, H. J. M., \& Vendrick, A. J. H. (1970). Simultaneous contrast, filling-in process and information processing in man's visual system. Experimental Brain Research, 11, 411-430.

Gouras, P., \& KRÜGER, J. (1979). Responses of cells in foveal visual cortex of the monkey to pure color contrast. Journal of Neurophysiology, 42, 850-860.

Grinvald, A., Leike, E., Frostig, R. D., Gilbert, C. D., \& Wiesel, T. N. (1986). Functional architecture of cortex revealed by optical imaging of intrinsic signals. Nature, 324, 361-364.

GrossBerg, S. (1983). The quantized geometry of visual space: The coherent computation of depth, form, and lightness. Behavioral \& Brain Sciences, 6, 625-692.

Grossberg, S. (1987a). Cortical dynamics of three-dimensional form, color, and brightness perception: I. Monocular theory. Perception \& Psychophysics, 41, 87-116.

Grossberg, S. (1987b). Cortical dynamics of three-dimensional form, color, and brightness perception: II. Binocular theory. Perception \& Psychophysics, 41, 117-158.

Grossberg, S., \& Mingolla, E. (1985a). Neural dynamics of form perception: Boundary completion, illusory figures, and neon color spreading. Psychological Review, 92, 173-211.

Grossberg, S., \& Mingolla, E. (1985b). Neural dynamics of perceptual grouping: Textures, boundaries, and emergent segmentations. Perception \& Psychophysics, 38, 141-171.

Grossberg, S., \& Mingolla, E. (1987). Neural dynamics of surface perception: Boundary webs, illuminants, and shape-from-shading. Computer Vision, Graphics, \& Image Processing, 37, 116-165.

Grossberg, S., \& Mingolla, E. (1988). The role of illusory contours in visual segmentation. In S. Petry \& G. Meyer (Eds.), The perception of illusory contours. New York: Springer-Verlag.

Growney, R. L. . NERI, D. F. (1986). The appearance of the Cornsweet illusion: Measures of perceived contrast and evenness of brightness. Perception \& Psychophysics, 39, 81-86.

Hamada, J. (1984). A multistage model for border contrast. Biological Cybernetics, 51, 65-70.
Heggelund, P. (1981a). Receptive field organisation of simple cells in cat striate cortex. Experimental Brain Research, 42, 89-98.

HegGelund, P. (1981b). Receptive field organisation of complex cells in cat striate cortex. Experimental Brain Research, 42, 99-107.

HEGGELUND, P. (1985). Quantitative studies of enhancement and suppression zones in the receptive field of simple cells in cat striate cortex. Journal of Physiology, 373, 293-310.

Heinemann, E. G. (1972). Simultaneous brightness induction. In D. Jameson \& L. M. Hurvich (Eds.), Handbook of sensory physiology: Vol. VII/4. Visual psychophysics (pp. 146-169). Berlin: SpringerVerlag.

Helson, H. (1963). Studies of anomalous contrast and assimilation. Journal of the Optical Society of America, 53, 179-184.

Hess, C., \& Pretori, H. (1894). Messende Untersuchungen über die Gesetzmässigkeit des simultanen Helligkeitscontrastes. Albrecht v. Graefes Archiv für Ophthalmologie, 40, 1-24.

HorN, B. K. P. (1974). Determining lightness from an image. Computer Graphics \& Image Processing, 3, 277-299.

Hubel, D. H., \& Wiesel, T. N. (1962). Receptive fields, binocular interaction and functional architecture in the cat's visual cortex. Joumal of Physiology, 160, 106-154.

Hubel, D. H., \& Wiesel, T. N. (1968). Receptive fields and functional architecture of monkey striate cortex. Journal of Physiology, 195, 215-243.

Hurvich, L. M. (1981). Color vision. Sunderland, MA: Sinauer.

JuLEsZ, B. (1971). Foundations of cyclopean perception. Chicago: University of Chicago Press.

Kanizsa, G., \& Minguzzi, G. F. (1986). An anomalous brightness differentiation. Perception, 15, 223-226.

Kato, H., Bishop, P. O., \& Orban, G. A. (1978). Hypercomplex and simple/complex cell classifications in cat striate cortex. Journal of Neurophysiology, 41, 1071-1095.

KATZ, D. (1935). The world of colour. London: Kegan Paul, Trench, Trubner.

KoFFKA, K. (1935). Principles of Gestalt psychology. New York: Harcourt \& Brace.

KRAUSKOPF, J. (1963). Effect of retinal image stabilization on the appearance of heterochromatic targets. Journal of the Optical Society of America, 53, 741-744.

LAMING, D. (1983). On the need for discipline in the construction of psychological theories. Behavioral \& Brain Sciences, 6, 669-670.

LAND, E. (1977). The retinex theory of color vision. Scientific American, 237, 108-128.

LAND, E. (1986). Recent advances in retinex theory. Vision Research, 26, 7-21.

LAND, E. MCCANN, J. J. (1971). Lightness and retinex theory. Journal of the Optical Society of America, 61, 1-11.

McCourt, M. E. (1982). A spatial frequency dependent gratinginduction effect. Vision Research, 22, 119-134.

Metell, F. (1974). The perception of transparency. Scientific American, 230, 90-98.

MetzGer, W. (1930). Optische Untersuchungen am Ganzfeld: II. Zur Phänomenologie des homogenen Ganzfeldes. Psychologische Forschung, 13, 6-29.

NIELSEN, D. E. (1985). Retinocortical wiring of the simple cells of the visual cortex. In D. Rose \& V. G. Dobson (Eds.), Models of the visual cortex. New York: Wiley.

NothduRFT, H. C., LEE, B. B. (1982). Responses to colored patterns in the macaque lateral geniculate nucleus: Pattern processing in single neurons. Experimental Brain Research, 48, 43-54.

Penrose, L. S., \& Penrose, R. (1958). Impossible objects: A special type of visual illusion. British Journal of Psychology, 49, 31-33.

Piccolino, M., Neyton, J., \& Gerschenfeld, H. M. (1984). Decrease of gap junction permeability induced by dopamine and cyclic adenosine $3^{\prime}: 5^{\prime}$-monophosphate in horizontal cells of turtle retina. Journal of Neuroscience, 4, 2477-2488.

Pritchard, R. M. (1961). Stabilized images on the retina. Scientific American, 204, 72-78. 
RATLIFF, F. (1965). Mach bands: Quantitative studies on neural networks in the retina. New York: Holden-Day.

RatLifF, F., \& HarTune, H. U. (1959). The response of limulus optic nerve fibers to patterns of illumination on the receptor mosaic. Journal of General Physiology, 42, 1241-1255.

Ratuiff, F., \& SiRovich, L. (1978). Equivalence classes of visual stimuli. Vision Research, 18, 845-851.

SAGI, D., \& HoChSTEIN, S. (1985). Lateral inhibition between spatially adjacent spatial-frequency channels? Perception \& Psychophysics, 37, 315-322.

Schiller, P. H., Finlay, B. L., \& Volman, S. F. (1976). Quantitative studies of single-cell properties in monkey striate cortex: $\mathbf{I}$. Spatiotemporal organization of receptive fields. Journal of Neurophysiology, 39, 1288-1319.

Sekuler, R. (1974). Spatial vision. In P. H. Mussen \& M. R. Rosenzweig (Eds.), Annual Review of Psychology. Palo Alto: Annual Reviews.

Shapley, R. (1986). The importance of contrast for the activity of single neurons, the VEP and perception. Vision Research, 26, 45-61.

ShaPley, R., \& REID, R. C. (1986). Contrast and assimilation in the perception of brightness. Proceedings of the National Academy of Sciences USA, 82, 5983-5986.

SilLito, A. M. (1984). Functional considerations of the operation of GABAergic inhibitory processes in the visual cortex. In A. Peters \& E. G. Jones (Eds.), Cerebral cortex (Vol. 2A). New York: Plenum Press.

Spitzer, H., \& Hochstein, S. (1985). A complex-cell receptive field model. Journal of Neurophysiology, 53, 1266-1286.

TANaKa, M., Lee, B. B., \& Creutzfeldt, O. D. (1983). Spectral tuning and contour representation in area 17 of the awake monkey. In J. D. Mollon \& L. T. Sharpe (Eds.), Colour vision. New York: Academic Press.

TODOROvić, D. (1983). Brightness perception and the Craik-O'BrienCornsweet effect. Unpublished master's thesis, University of Connecticut, Storrs.

Todorović, D. (1987). The Craik-O'Brien-Cornsweet effect: New varieties and their theoretical implications. Perception \& Psychophysics, 42, 545-560.

WALLACH, H. (1948). Brightness constancy and the nature of achromatic colors. Journal of Experimental Psychology, 38, 310-324.

Wallach, H. (1976). On perception. New York: Quadrangle.

WALLS, G. (1954). The filling-in process. American Journal of Optometry, 31, 329-340.

WhITE, M. (1979). A new effect of pattern on perceived lightness. Perception, 8, 413-416.

Yarbus, A. L. (1967). Eye movements and vision. New York: Plenum Press.

YUND, E. W., \& ARMington, J. C. (1975). Color and brightness contrast effects as a function of spatial variables. Vision Research, 15, 917-930.

\section{APPENDIX}

The equations underlying the model are based on and are an extension of work by Grossberg (1983), Cohen and Grossberg (1984), and Grossberg and Mingolla (1985b, 1987a). The exposition follows the description of levels in Figure 2 . Only the $2-D$ versions of the equations are presented.The $1-D$ forms can be derived by straightforward simplifications. The 2-D simulations were performed either on a $16 \times 16$ lattice of units (the yinyang square), a $30 \times 30$ lattice (simulations in Part 5-The COCE with and without... and Percept of an impossible staircase ...), or a $40 \times 40$ lattice (all other simulations). The one-dimensional simulations involve 256 units.

\section{Level 1}

We denote by $I_{i j}$ the value of the stimulus input at position $(i, j)$ in the lattice. In all simulations these values varied between
1 and 9. To compute the spatial convolutions of Level 2 cells without causing spurious edge effects at the extremities of the luminance profile, the luminance values at the extremities were continued outward as far as necessary.

\section{Level 2}

The activity $x_{i j}$ of a Level 2 on-cell at position $(i, j)$ of the lattice obeys a membrane equation

$$
\frac{d}{d t} x_{i j}=-A x_{i j}+\left(B-x_{i j}\right) C_{i j}-\left(x_{i j}+D\right) E_{i j},
$$

where $C_{i j}\left(E_{i j}\right)$ is the total excitatory (inhibitory) input to $x_{i j}$. Each input $C_{i j}$ and $E_{i j}$ is a discrete convolution with Gaussian kernel of the inputs $I_{p q}$ :

$$
C_{i j}=\sum_{(p, q)} I_{p q} C_{p q i j}
$$

and

$$
E_{i j}=\sum_{(p, q)} I_{p q} E_{p q i j}
$$

where

$$
C_{p q i j}=C \exp \left\{-\alpha^{-2} \log 2\left[(p-i)^{2}+(q-j)^{2}\right]\right\}
$$

and

$$
E_{\rho q i j}=E \exp \left\{-\beta^{-2} \log 2\left[(p-i)^{2}+(q-j)^{2}\right]\right\} .
$$

Thus, the influence exerted on the Level 2 potential $x_{i j}$ by input $I_{p q}$ diminishes with increasing distance between the two corresponding locations. The decrease is isotropic, inducing the circular shape of the receptive fields. To achieve an on-center off-surround anatomy, coefficient $C$ of the excitatory kernel in Equation A4 is chosen larger than coefficient $E$ of the inhibitory kernel in Equation A5, but $\alpha$, the radius of the excitatory spread at half strength in Equation A4, is chosen smaller than $\beta$, its inhibitory counterpart in Equation A5. In the simulations, this equation is solved at equilibrium. Then $(d / d t) x_{i j}=0$, so that

$$
x_{i j}=\frac{\sum_{(p, q)}\left(B C_{p q i j}-D E_{p q i j}\right) I_{p q q}}{A+\sum_{(p, q)}\left(C_{p q i j}+E_{p q i j}\right) I_{p q}} .
$$

The denominator term normalizes the activity $x_{i j}$.

The off-cell potential $\bar{x}_{i j}$ at position $(i, j)$ also obeys a membrane equation with an equilibrium value of the same form

$$
\bar{x}_{i j}=\frac{\sum_{(p, q)}\left(B \bar{C}_{p q i j}-D \bar{E}_{p q i j}\right) I_{p q}}{A+\sum_{(p, q)}\left(\bar{C}_{p q i j}+\bar{E}_{p q i j}\right) I_{p q}} .
$$

The duality between on-cell and off-cell receptive fields was achieved by setting

$$
\bar{C}_{p q i j}=E_{p q i j}
$$

and

$$
\bar{E}_{p q i j}=C_{p q i j} .
$$

The output signal from Level 2 is the nonnegative, or rectified, part of $x_{i j}$ :

$$
X_{i j}=\max \left(x_{i j}, 0\right)
$$

Level 3

The potential $y_{i j k}$ of the cell centered at position $(i, j)$ with 
orientation $k$ on the hour code in Figure 7 obeys an additive equation

$$
\frac{d}{d t} y_{i j k}=-y_{i j k}+\sum_{(p, q)} X_{p q} F_{p q i j}^{(k)}
$$

which is computed at equilibrium

$$
y_{i j k}=\sum_{(p, q)} X_{p q} F_{p q i j}^{(k)}
$$

in all our simulations. To generate an oriented kernel $F_{p q i j}^{(k)}$ as simply as possible, let $F_{p q i j}^{(k)}$ be the difference of an isotropic kernel $G_{p q i j}$ centered at $(i, j)$ and another isotropic kernel $H_{p q i j}^{(k)}$ whose center $\left(i+m_{k}, j+n_{k}\right)$ is shifted from $(i, j)$ as follows:

$$
F_{p q i j}^{(k)}=G_{p q i j}-H_{p q i j}^{(k)},
$$

where

$$
G_{p q i j}=\exp \left\{-\gamma^{-2}\left[(p-i)^{2}+(q-j)^{2}\right]\right\}
$$

and

$$
H_{p q i j}^{(k)}=\exp \left\{-\gamma^{-2}\left[\left(\mathrm{p}-i-m_{k}\right)^{2}+\left(q-j-n_{k}\right)^{2}\right]\right\},
$$

with

$$
m_{k}=\sin \frac{2 \pi k}{K}
$$

and

$$
n_{k}=\cos \frac{2 \pi k}{K}
$$

In the 2-D simulations, the number $K$ of hour codes is 12, whereas for the 1-D simulations it is 2 .

The output signal from Level 3 to Level 4 is the nonnegative, or rectified, part of $y_{i j k}$, namely

$$
Y_{i j k}=\max \left(y_{i j k}, 0\right) \text {. }
$$

\section{Level 4}

Each Level 4 potential $z_{i j k}$ with position $(i, j)$ and orientation $k$ is made sensitive to orientation but insensitive to direction of contrast by summing the output signals from the appropriate pair of Level 3 units with opposite contrast sensitivities; viz.,

$$
z_{i j k}=Y_{i j k}+Y_{i j[k+(K / 2)]} \text {. }
$$

An output signal $Z_{i j k}$ is generated from Level 4 to Level 5 if the activity $z_{i j k}$ exceeds the threshold $L$ :

$$
Z_{i j k}=\max \left(z_{i j k}-L, 0\right) \text {. }
$$

\section{Level 5}

A Level 5 signal $z_{i j}$ at position $(i, j)$ is the sum of output signals from all Level 4 units at that position; viz.,

$$
Z_{i j}=\sum_{k} Z_{i j k}
$$

\section{Level 6}

Each potential $S_{i j}$ at position $(i, j)$ of the syncytium obeys a nonlinear diffusion equation

$$
\frac{d}{d t} S_{i j}=-M S_{i j}+\sum_{(p, q) \in N_{i j}}\left(S_{p q}-S_{i j}\right) P_{p q i j}+X_{i j}
$$

The diffusion coefficients that regulate the magnitude of cross influence of location $(i, j)$ with location $(p, q)$ depend on the BC signals $Z_{p q}$ and $Z_{i j}$ as follows:

$$
P_{p q i j}=\frac{\delta}{1+\epsilon\left(Z_{p q}+Z_{i j}\right)} .
$$

The set $N_{i j}$ of locations comprises only the lattice nearest neighbors of $(i, j)$ :

$$
N_{i j}=\{(i, j-1),(i-1, j),(i+1, j),(i, j+1)\} .
$$

At lattice edges and corners, this set is reduced to the set of existing neighbors. According to Equation A22, each potential $S_{i j}$ is activated by the on-cell output signal $X_{i j}$ and thereupon engages in passive decay (term $-M S_{i j}$ ) and diffusive filling-in with its four nearest neighbors to the degree permitted by the diffusion coefficients $P_{p q i j}$. At equilibrium, each $S_{i j}$ is computed as the solution of a set of simultaneous equations

$$
S_{i j}=\frac{X_{i j}+\sum_{(p, q) \in N_{u}} S_{p q} P_{p q i j}}{M+\sum_{(p, q) \in N_{U}} P_{p q i j}},
$$

which is compared with properties of the brightness percept. In all simulations, the following parameter values were used: $A=1, B=90, D=60, \gamma=1$. Due to differences in dimensionality and scale, several parameters were given different values across sets of simulations. All 2-D simulations shared the following parameters: $C=18, M=1, \alpha=.25, \epsilon=1$. The yin-yang square $(16 \times 16)$ simulations used $E=1, \beta=2, \delta=100, L=15$. All other 2-D $(30 \times 30$ and $40 \times 40)$ simulations used $E=.5, \beta=3$, $\delta=300, L=10$. All 1-D simulations used $C=4, M=10, \alpha=1$, $\epsilon=100, E=.5, \beta=8, \delta=100,000, L=5$.

Finally, to illustrate the effects of parameter changes, the following values were different from the ones listed above. In Figure $6 \mathrm{e}, \gamma=3$. In Figures $16 \mathrm{c}$ and $16 \mathrm{~d}, \epsilon=1,000$. In Figures $16 \mathrm{a}$ and $16 \mathrm{~b}$, the $\mathrm{BC}$ signal $Z_{i}$ was transformed through the sigmoid function $10 Z_{i}^{5}\left(1+Z_{i}^{5}\right)^{-1}$. 